ALEA, Lat. Am. J. Probab. Math. Stat. 13, 863-923 (2016)

DOI: 10.30757/ALEA.v13-34

\title{
Renewal structure and local time for diffusions in random environment
}

\author{
Pierre Andreoletti, Alexis Devulder and Grégoire Véchambre \\ Université d'Orléans, Laboratoire MAPMO - Fédération Denis Poisson, Bâtiment de \\ mathématiques - Rue de Chartres B.P. 6759 - 45067 Orléans cedex 2, France \\ E-mail address: Pierre.Andreoletti@univ-orleans.fr \\ Laboratoire de Mathématiques de Versailles, UVSQ, CNRS, Université Paris-Saclay, 78035 \\ Versailles, France. \\ E-mail address: devulder@math.uvsq.fr \\ Université d'Orléans, Laboratoire MAPMO - Fédération Denis Poisson, Bâtiment de \\ mathématiques - Rue de Chartres B.P. 6759 - 45067 Orléans cedex 2, France \\ E-mail address: Gregoire.Vechambre@ens-rennes.fr
}

\begin{abstract}
We study a one-dimensional diffusion $X$ in a drifted Brownian potential $W_{\kappa}$, with $0<\kappa<1$, and focus on the behavior of the local times $(\mathcal{L}(t, x), x)$ of $X$ before time $t>0$. In particular we characterize the limit law of the supremum of the local time, as well as the position of the favorite site. These limits can be written explicitly from a two dimensional stable Lévy process. Our analysis is based on the study of an extension of the renewal structure which is deeply involved in the asymptotic behavior of $X$.
\end{abstract}

\section{Introduction}

1.1. Presentation of the model. Let $(X(t), t \geq 0)$ be a diffusion in a random càdlàg potential $(V(x), x \in \mathbb{R})$, defined informally by $X(0)=0$ and

$$
\mathrm{d} X(t)=\mathrm{d} \beta(t)-\frac{1}{2} V^{\prime}(X(t)) \mathrm{d} t
$$

where $(\beta(s), s \geq 0)$ is a Brownian motion independent of $V$. Rigorously, $X$ is defined by its conditional generator given $V$,

$$
\frac{1}{2} e^{V(x)} \frac{\mathrm{d}}{\mathrm{d} x}\left(e^{-V(x)} \frac{\mathrm{d}}{\mathrm{d} x}\right) .
$$

Received by the editors September 5, 2015; accepted April 26, 2016.

2010 Mathematics Subject Classification. 60K37, 60J55, 60J60, 60K05.

Key words and phrases. Diffusion, random potential, renewal process, local time.

This research was partially supported by the French ANR project MEMEMO2 2010 BLAN 0125 . 
We put ourselves in the case where $V$ is a negatively drifted Brownian motion: $V(x)=W_{\kappa}(x):=W(x)-\frac{\kappa}{2} x, x \in \mathbb{R}$, with $0<\kappa<1$ and $(W(x), x \in \mathbb{R})$ is a two sided Brownian motion. We explain at the end of Section 1.2 what should be done to extend our results to a more general Lévy potential.

We denote by $P$ the probability measure associated to $W_{\kappa}($.$) . The probability$ conditionally on the potential $W_{\kappa}$ is denoted by $\mathbb{P}^{W_{\kappa}}$ and is called the quenched probability. We also define the annealed probability as

$$
\mathbb{P}(.):=\int \mathbb{P}^{W_{\kappa}}(.) P\left(W_{\kappa} \in \mathrm{d} \omega\right) .
$$

We denote respectively by $\mathbb{E}^{W_{\kappa}}, \mathbb{E}$, and $E$ the expectations with regard to $\mathbb{P}^{W_{\kappa}}, \mathbb{P}$ and $P$. In particular, $X$ is a Markov process under $\mathbb{P}^{W_{\kappa}}$ but not under $\mathbb{P}$.

This diffusion $X$ has been introduced by Schumacher (1985). It is generally considered as a continuous time analogue of random walks in random environment (RWRE). We refer e.g. to Zeitouni (2004) for general properties of RWRE.

In our case, since $\kappa>0$, the diffusion $X$ is a.s. transient and its asymptotic behavior was first studied by Kawazu and Tanaka: if $H(r)$ is the hitting time of $r \in \mathbb{R}$ by $X$,

$$
H(r):=\inf \{s>0, X(s)=r\},
$$

Kawazu and Tanaka (1997) proved that, for $0<\kappa<1$ under the annealed probability $\mathbb{P}, H(r) / r^{1 / \kappa}$ converges in law as $r \rightarrow+\infty$ to a $\kappa$-stable distribution (see also Hu et al., 1999, and Tanaka, 1997). Here we are interested in the local time of $X$, which is the jointly continuous process $(\mathcal{L}(t, x), t>0, x \in \mathbb{R})$ satisfying, for any positive measurable function $f$,

$$
\int_{0}^{t} f(X(s)) \mathrm{d} s=\int_{-\infty}^{+\infty} f(x) \mathcal{L}(t, x) \mathrm{d} x, \quad t>0 .
$$

One quantity of particular interest is the supremum of the local time of $X$ at time $t$, defined as

$$
\mathcal{L}^{*}(t):=\sup _{x \in \mathbb{R}} \mathcal{L}(t, x), \quad t>0 .
$$

For Brox's diffusion, that is, for the diffusion $X$ in the recurrent case $\kappa=0$, it is proved in Andreoletti and Diel (2011) that the local time process until time $t$ re-centered at the localization coordinate $b_{t}$ (see Brox, 1986) and renormalized by $t$ converges in law under the annealed probability $\mathbb{P}$. This allows the authors of Andreoletti and Diel (2011) to derive the limit law of the supremum of the local time at time $t$ as $t \rightarrow+\infty$. We recall their result below in order to compare it with the results of the present paper. To this aim, we introduce for every $\kappa \geq 0$,

$$
\mathcal{R}_{\kappa}:=\int_{0}^{+\infty} e^{-W_{\kappa}^{\uparrow}(x)} \mathrm{d} x+\int_{0}^{+\infty} e^{-\widetilde{W}_{\kappa}^{\uparrow}(x)} \mathrm{d} x,
$$

where $\left(W_{\kappa}^{\uparrow}(x), x \geq 0\right)$ and $\left(\widetilde{W}_{\kappa}^{\uparrow}, x \geq 0\right)$ are two independent copies of the process $\left(W_{\kappa}(x), x \geq 0\right)$ Doob-conditioned to remain positive.

Theorem 1.1. (Andreoletti and Diel, 2011) If $\kappa=0$, then

$$
\frac{\mathcal{L}^{*}(t)}{t} \stackrel{\text { 自 }}{\rightarrow} \frac{1}{\mathcal{R}_{\kappa}},
$$

where $\stackrel{\text { II }}{\rightarrow}$ denotes convergence in law under the annealed probability $\mathbb{P}$ as $t \rightarrow+\infty$. 
Extending their approach, and following the results of Shi (1998), Diel (2011) obtains the non-trivial normalizations for the almost sure behavior of the lim sup and the $\liminf$ of $\mathcal{L}^{*}(t)$ as $t \rightarrow+\infty$ when $\kappa=0$. Notice that corresponding results have been previously established in Dembo et al. (2007) and Gantert et al. (2010) for the discrete analogue of $X$ in the recurrent case $\kappa=0$, the recurrent RWRE generally called Sinai's random walk.

One of our aims in this paper is to extend the study of the local time of $X$ in the case $0<\kappa<1$, and deduce from that the weak asymptotic behavior of $\mathcal{L}^{*}(t)$ suitably renormalized as $t \rightarrow+\infty$.

Before going any further, let us recall to the reader what is known for the slow transient cases. For transient RWRE in the case $0<\kappa \leq 1$ (see Kesten et al., 1975 for the seminal paper), a result of Gantert and Shi (2002) states the almost sure behavior for the limsup of the supremum of the local time $\mathcal{L}_{S}^{*}(n)$ of these random walks (denoted by $S$ ) at time $n$ : there exists a constant $c>0$ such that $\lim \sup _{n \rightarrow+\infty} \mathcal{L}_{S}^{*}(n) / n=c>0 \mathbb{P}$ almost surely. Contrarily to the recurrent case (Gantert et al., 2010) their method, based on a relationship between the RWRE $S$ and a branching process in random environment, cannot be exploited to determine the limit law of $\mathcal{L}_{S}^{*}(n) / n$.

For the transient diffusion $X$ considered here, the only paper dealing with $\mathcal{L}^{*}(t)$ is Devulder $(2016+)$, in which it is proved, among other results, that when $0<\kappa<1$, $\lim \sup _{t \rightarrow+\infty} \mathcal{L}^{*}(t) / t=+\infty$ almost surely. But once again his method cannot be used to characterize the limit law of $\mathcal{L}^{*}(t) / t$ in the case $0<\kappa<1$.

Our motivation here is twofold, first we prove that our approach enables to characterize the limit law of $\mathcal{L}^{*}(t) / t$ and open a way to determine the correct almost sure behavior of $\mathcal{L}^{*}(t)$ as was done for Brox's diffusion by Shi (1998) and Diel (2011). Second we make a first step on a specific way to study the local time which could be used in estimation problems in random environment, see Adelman and Enriquez (2004), Andreoletti (2011), Andreoletti and Diel (2012), Andreoletti et al. (2015), Comets et al. (2016+), Comets et al. (2014), Falconnet et al. (2014).

The method we develop here is an improvement of the one used in Andreoletti and Devulder (2015) about the localization of $X(t)$ for large $t$.

Before recalling the main result of this paper Andreoletti and Devulder (2015), we need to introduce some new objects. We start with the notion of $h$-extrema, with $h>0$, introduced by Neveu and Pitman (1989) and studied more specifically in our case of drifted Brownian motions by Faggionato (2009). For $h>0$, we say that $x \in \mathbb{R}$ is an h-minimum for a given continuous function $f, \mathbb{R} \rightarrow \mathbb{R}$, if there exist $u<x<v$ such that $f(y) \geq f(x)$ for all $y \in[u, v], f(u) \geq f(x)+h$ and $f(v) \geq f(x)+h$. Moreover, $x$ is an $h$-maximum for $f$ iff $x$ is an $h$-minimum for $-f$. Finally, $x$ is an $h$-extremum for $f$ iff it is an $h$-maximum or an $h$-minimum for $f$.

As we are interested in the diffusion $X$ until time $t$ for large $t$, we only focus on the $h_{t}$-extrema of $W_{\kappa}$, where

$$
h_{t}:=\log t-\phi(t), \quad \text { with } 0<\phi(t)=o(\log t), \quad \log \log t=o(\phi(t)),
$$

and $t \mapsto \phi(t)$ is an increasing function, as in Andreoletti and Devulder (2015). It is known (see Faggionato, 2009) that almost surely, the $h_{t}$-extrema of $W_{\kappa}$ form a sequence indexed by $\mathbb{Z}$, unbounded from below and above, and that the $h_{t^{-}}$ minima and $h_{t}$-maxima alternate. We denote respectively by $\left(m_{j}, j \in \mathbb{Z}\right)$ and $\left(M_{j}, j \in \mathbb{Z}\right)$ the increasing sequences of $h_{t}$-minima and of $h_{t}$-maxima of $W_{\kappa}$, such 
that $m_{0} \leq 0<m_{1}$ and $m_{j}<M_{j}<m_{j+1}$ for every $j \in \mathbb{Z}$. Define

$$
N_{t}:=\max \left\{k \in \mathbb{N}, \sup _{0 \leq s \leq t} X(s) \geq m_{k}\right\}
$$

the number of (positive) $h_{t}$-minima on $\mathbb{R}_{+}$visited by $X$ until time $t$. We have the following result.

Theorem 1.2. (Andreoletti and Devulder, 2015) Assume $0<\kappa<1$. There exists a constant $\mathcal{C}_{1}>0$, such that

$$
\lim _{t \rightarrow+\infty} \mathbb{P}\left(\left|X(t)-m_{N_{t}}\right| \leq \mathcal{C}_{1} \phi(t)\right)=1
$$

This result proves that before time $t$, the diffusion $X$ visits the $N_{t}$ leftmost positive $h_{t}$-minima, and then gets stuck in a very small neighborhood of an ultimate one, which is $m_{N_{t}}$. An analogous result was proved for transient RWRE in the zero speed regime $0<\kappa<1$ by Enriquez et al. (2009a). This phenomenon is due to two facts: the first one is the appearance of a renewal structure which is composed of the times it takes the process to move from one $h_{t}$-minimum to the following one. The second is the fact that like in Brox's case $\kappa=0$, the process is trapped a significant amount of time in the neighborhood of the local minimum $m_{N_{t}}$.

It is the extension of this renewal structure to the sequence of local times at the $h_{t}$-minima that we study here. We now detail our results.

1.2. Results. Let us introduce some notation involved in the statement of our results. Assume that $0<\kappa<1$.

Denote by $\left(D\left([0,+\infty), \mathbb{R}^{2}\right), J_{1}\right)$ the space of càdlàg functions $[0,+\infty) \rightarrow \mathbb{R}^{2}$ with $J_{1}$-Skorokhod topology and denote by $\stackrel{\mathbf{Z L}_{S}}{\rightarrow}$ the convergence in law for this topology. On this space, define a 2 -dimensional Lévy process $\left(\mathcal{Y}_{1}, \mathcal{Y}_{2}\right)$ taking values in $\mathbb{R}_{+} \times \mathbb{R}_{+}$, which is a pure positive jump process with $\kappa$-stable Lévy measure $\nu$ given by

$\forall x>0, \forall y>0, \quad \nu\left(\left[x,+\infty\left[\times\left[y,+\infty[)=\frac{\mathcal{C}_{2}}{y^{\kappa}} \mathbb{E}\left[\left(\mathcal{R}_{\kappa}\right)^{\kappa} \mathbb{1}_{\mathcal{R}_{\kappa} \leq \frac{y}{x}}\right]+\frac{\mathcal{C}_{2}}{x^{\kappa}} \mathbb{P}\left(\mathcal{R}_{\kappa}>\frac{y}{x}\right)\right.\right.\right.\right.$,

where $\mathcal{R}_{\kappa}$ is defined in (1.2) and $\mathcal{C}_{2}$ is a positive constant (see Lemma 4.1). The Laplace transform of $\mathcal{R}_{\kappa}$ is given by

$$
E\left(e^{-\gamma \mathcal{R}_{\kappa}}\right)=\left(\frac{(2 \gamma)^{\kappa / 2}}{\kappa \Gamma(\kappa) I_{\kappa}(2 \sqrt{2 \gamma})}\right)^{2} \quad \gamma>0
$$

as proved in Lemma 6.6 below, where $I_{\kappa}$ is the modified Bessel function of the first kind of index $\kappa$. Moreover, $\mathcal{R}_{\kappa}$ admits moments of any positive order (see also Lemma 6.6). In particular $\mathbb{E}\left[\left(\mathcal{R}_{\kappa}\right)^{\kappa}\right]$ is finite and $\nu$ is well defined.

For a given càdlàg function $f$ in $D([0,+\infty), \mathbb{R})$, define for any $s>0, a>0$ :

$$
f^{\natural}(s):=\sup _{0 \leq r \leq s}\left(f(r)-f\left(r^{-}\right)\right), \quad f^{-1}(a):=\inf \{x \geq 0, f(x)>a\},
$$

where $f\left(r^{-}\right)$denotes the left limit of $f$ at $r$. In words, $f^{\natural}(s)$ is the largest jump of $f$ before time $s$, whereas $f^{-1}(a)$ is the first time $f$ is strictly larger than $a$. We also 
introduce the couple of random variables $\left(\mathcal{I}_{1}, \mathcal{I}_{2}\right)$ as follows,

$$
\mathcal{I}_{1}:=\mathcal{Y}_{1}^{\natural}\left(\mathcal{Y}_{2}^{-1}(1)^{-}\right), \quad \mathcal{I}_{2}:=\left(1-\mathcal{Y}_{2}\left(\mathcal{Y}_{2}^{-1}(1)^{-}\right)\right) \times \frac{\mathcal{Y}_{1}\left(\mathcal{Y}_{2}^{-1}(1)\right)-\mathcal{Y}_{1}\left(\mathcal{Y}_{2}^{-1}(1)^{-}\right)}{\mathcal{Y}_{2}\left(\mathcal{Y}_{2}^{-1}(1)\right)-\mathcal{Y}_{2}\left(\mathcal{Y}_{2}^{-1}(1)^{-}\right)}
$$

We recall that $\stackrel{\text { I }}{\rightarrow}$ denotes convergence in law under the annealed probability $\mathbb{P}$ as $t \rightarrow+\infty$. We are now ready to state our first result.

Theorem 1.3. Assume $0<\kappa<1$. We have,

$$
\frac{\mathcal{L}^{*}(t)}{t} \stackrel{\text { I }}{\rightarrow} \mathcal{I}=: \max \left(\mathcal{I}_{1}, \mathcal{I}_{2}\right)
$$

Contrary to the recurrent case $\kappa=0$, we have no scaling property for the potential, and the diffusion $X$ cannot be localized in a single valley as we can see in Theorem 1.2. However in the transient case we can make appear and use a renewal structure.

We now give an intuitive interpretation of this theorem, explaining the appearance of the Lévy process $\left(\mathcal{Y}_{1}, \mathcal{Y}_{2}\right)$.

First for any $s>0, \mathcal{Y}_{1}(s)$ is the limit of the sum of the first $\left\lfloor s e^{\kappa \phi(t)}\right\rfloor$ normalized (by $t$ ) local times taken specifically at the $\left\lfloor s e^{\kappa \phi(t)}\right\rfloor$ first $h_{t}$-minima (see Proposition 1.4 below). Similarly, $\mathcal{Y}_{2}(s)$ is the limit of the sum of the exit times of the $\left\lfloor s e^{\kappa \phi(t)}\right\rfloor$ first $h_{t}$-valleys, normalized (by $t$ ), where an $h_{t}$-valley is a large neighborhood of an $h_{t}$-minimum. For a rigorous definition of these $h_{t}$-valleys, see Section 2.2 and Figure 2.1.

So, by definition, $\mathcal{I}_{1}$ is the largest jump of the process $\mathcal{Y}_{1}$ before the first time $\mathcal{Y}_{2}$ is larger than 1. It can be interpreted as the largest (re-normalized) local time among the local times at the $h_{t}$-minima visited by $X$ until time $t$ and from which $X$ has already escaped. That is to say, $\mathcal{I}_{1}$ is the limit of the random variable $\sup _{k \leq N_{t}-1} \mathcal{L}\left(m_{k}, t\right) / t$.

$\mathcal{I}_{2}$ is a product of two factors: the first one, $\left(1-\mathcal{Y}_{2}\left(\mathcal{Y}_{2}^{-1}(1)^{-}\right)\right)$, corresponds to the (re-normalized) amount of time left to the diffusion $X$ before time $t$ after it has reached the ultimate visited $h_{t}$-minimum $m_{N_{t}}$, that is, to $\left(t-H\left(m_{N_{t}}\right)\right) / t$. The second factor corresponds to the local time of $X$ at this ultimate $h_{t}$-minimum $m_{N_{t}}$, that is to say $\mathcal{I}_{2}$ is the limit of $\mathcal{L}\left(t, m_{N_{t}}\right) / t$. Intuitively $\mathcal{Y}_{2}$ is built from $\mathcal{Y}_{1}$ by multiplying each of its jumps by an independent copy of the variable $\mathcal{R}_{\kappa}$. Therefore this second factor can be seen as an independent copy of $1 / \mathcal{R}_{\kappa}$ taken at the instant of the overshoot of $\mathcal{Y}_{2}$ which makes it larger than 1. Notice that this variable $\mathcal{R}_{\kappa}$ plays a similar role as $\mathcal{R}_{0}$ of Theorem 1.1. Indeed as in the case $\kappa=0$, the diffusion $X$ is prisoner in the neighborhood of the last $h_{t}$-minimum visited before time $t$.

We prove Theorem 1.3 by showing first that portions of the trajectory of $X$ re-centered at the local $h_{t}$-minima, until time $t$, are made (in probability) with independent parts. This has been partially proved in Andreoletti and Devulder (2015) but we have to improve their results and add simultaneously the study of the local time.

Second, we prove that the supremum of the local time is, mainly, a function of the sum of theses independent parts, which converges to a Lévy process. We now provide some details about this. 
Recall that $\left(W_{\kappa}^{\uparrow}(s), s \geq 0\right)$ is defined as a continuous process, taking values in $\mathbb{R}_{+}$, with infinitesimal generator given for every $x>0$ by

$$
\frac{1}{2} \frac{\mathrm{d}^{2}}{\mathrm{~d} x^{2}}+\frac{\kappa}{2} \operatorname{coth}\left(\frac{\kappa}{2} x\right) \frac{\mathrm{d}}{\mathrm{d} x} \text {. }
$$

This process $W_{\kappa}^{\uparrow}$ can be thought of as a $(-\kappa / 2)$-drifted Brownian motion $W_{\kappa}$ Doobconditioned to stay positive, with the terminology of Bertoin (1996), which is called Doob conditioned to reach $+\infty$ before 0 in Faggionato (2009) (for more details, see Section 2.1 in Andreoletti and Devulder (2015), where $W_{\kappa}^{\uparrow}$ is denoted by $R$ ). We call $\operatorname{BES}(3, \kappa / 2)$ the law of $\left(W_{\kappa}^{\uparrow}(s), s \geq 0\right)$. That is, $\left(W_{\kappa}^{\uparrow}(s), s \geq 0\right)$ is a 3 -dimensional $(\kappa / 2)$-drifted Bessel process starting from 0 . For any process $\left(U(t), \quad t \in \mathbb{R}_{+}\right)$, we denote by

$$
\tau^{U}(a):=\inf \{t>0, U(t)=a\},
$$

the first time this process hits $a$, with the convention $\inf \emptyset=+\infty$. For $a<b$, $\left(W_{\kappa}^{b}(s), 0 \leq s \leq \tau^{W_{\kappa}^{b}}(a)\right)$ is defined as a $(-\kappa / 2)$-drifted Brownian motion starting from $b$ and killed when it first hits $a$. We now introduce some functionals of $W_{\kappa}$ and $W_{\kappa}^{\uparrow}$, which already appeared in Andreoletti and Devulder (2015, Section 4.1):

$$
\begin{aligned}
F^{ \pm}(x) & :=\int_{0}^{\tau^{W_{\kappa}^{\uparrow}}(x)} \exp \left( \pm W_{\kappa}^{\uparrow}(s)\right) \mathrm{d} s, & x>0, \\
G^{ \pm}(a, b) & :=\int_{0}^{\tau^{W_{\kappa}^{b}}(a)} \exp \left( \pm W_{\kappa}^{b}(s)\right) \mathrm{d} s, & a<b .
\end{aligned}
$$

Let $0<\delta<1$, define

$$
n_{t}:=\left\lfloor e^{\kappa \phi(t)(1+\delta)}\right\rfloor, \quad t>0,
$$

which is, with large probability, an upper bound for $N_{t}$ as stated in Lemma 3.1.

Let $\left(S_{j}, R_{j}, \mathbf{e}_{j}, j \leq n_{t}\right)$ be a sequence of i.i.d. random variables depending on $t$, with $S_{j}, R_{j}$ and $\mathbf{e}_{\mathbf{j}}$ independent, $S_{1} \stackrel{\text { 年 }}{=} F^{+}\left(h_{t}\right)+G^{+}\left(h_{t} / 2, h_{t}\right), R_{1} \stackrel{\text { 妾 }}{=} F^{-}\left(h_{t} / 2\right)+$ $\tilde{F}^{-}\left(h_{t} / 2\right)$ and $\mathbf{e}_{1} \stackrel{\text { 近 }}{=} \mathcal{E}(1 / 2)$ (an exponential random variable with parameter $1 / 2$ ), where $\tilde{F}^{-}$is an independent copy of $F^{-}$and $F^{+}$is independent of $G^{+}$, and $\stackrel{\text { 近 }}{=}$ denotes equality in law. Define $\ell_{j}:=\mathbf{e}_{j} S_{j}$ and $\mathcal{H}_{j}:=\ell_{j} R_{j}$. Note that to simplify the notation, we do not make appear the dependence in $t$ in the sequel. Intuitively, $\ell_{j}$ plays the role of the local time at the $j$-th positive $h_{t}$-minimum $m_{j}$ if $X$ escapes from the $j$-th $h_{t}$-valley before time $t$, that is, if $j<N_{t}$. Similarly, $\mathcal{H}_{j}$ plays the role of the time $X$ spends in the $j$-th $h_{t}$-valley before escaping from it.

Define the family of processes $\left(Y_{1}, Y_{2}\right)^{t}$ indexed by $t$, by

$$
\forall s \geq 0, \quad\left(Y_{1}, Y_{2}\right)_{s}^{t}=\left(Y_{1}^{t}(s), Y_{2}^{t}(s)\right):=\frac{1}{t} \sum_{j=1}^{\left\lfloor s e^{\kappa \phi(t)}\right\rfloor}\left(\ell_{j}, \mathcal{H}_{j}\right) .
$$

Recall that $\stackrel{\mathbf{Z}_{S}}{\rightarrow}$ denotes convergence in law under $J_{1}$-Skorokhod topology. Here is our next result.

Proposition 1.4. Assume $0<\kappa<1$. We have under $\mathbb{P}$, as $t \rightarrow+\infty$,

$$
\left(Y_{1}, Y_{2}\right)^{t} \stackrel{\mathfrak{Z L}_{S}}{\rightarrow}\left(\mathcal{Y}_{1}, \mathcal{Y}_{2}\right)
$$


Once this is proved, we check that we can approximate, in law, the renormalized local time $\mathcal{L}^{*}(t) / t$ by a function of $\left(Y_{1}, Y_{2}\right)^{t}$. We obtain such an expression in Proposition 5.1. Then to obtain the limit claimed in Theorem 1.3, we prove the continuity (in $J_{1}$-topology) of the involved mapping and apply a continuous mapping Theorem (see Section 4.3).

It appears that with this method we can also obtain some other asymptotics. Indeed, we obtain in the following theorem the convergence in law of the supremum of the local time of $X$ before $X$ hits the last $h_{t}$-minimum $m_{N_{t}}$ visited before time $t$, of the supremum of the local time of $X$ before $X$ leaves the last $h_{t}$-valley visited before time $t$ (the one around $m_{N_{t}}$ ) approximately at time $H\left(m_{N_{t}+1}\right)$, and of the position of the favorite site.

Theorem 1.5. Assume $0<\kappa<1$. We have the following convergences in law under $\mathbb{P}$ as $t \rightarrow+\infty$,

$$
\begin{aligned}
\frac{\mathcal{L}^{*}\left(H\left(m_{N_{t}+1}\right)\right)}{t} & \stackrel{\text { I }}{\rightarrow} \mathcal{Y}_{1}^{\natural}\left(\mathcal{Y}_{2}^{-1}(1)\right), \\
\frac{\mathcal{L}^{*}\left(H\left(m_{N_{t}}\right)\right)}{t} & \stackrel{\text { I }}{\rightarrow} \quad \mathcal{Y}_{1}^{\natural}\left(\mathcal{Y}_{2}^{-1}(1)^{-}\right)=\mathcal{I}_{1} .
\end{aligned}
$$

Let us call $F_{t}^{*}$ the position of the first favorite site, that is, $F_{t}^{*}:=\inf \{x \in \mathbb{R}, \mathcal{L}(t, x)=$ $\left.\mathcal{L}^{*}(t)\right\}$. Then,

$$
\frac{F_{t}^{*}}{X(t)} \stackrel{\text { 近 }}{\rightarrow} \mathcal{B} U_{[0,1]}+1-\mathcal{B}
$$

where $\mathcal{B}$ is a Bernoulli random variable with parameter $\mathbb{P}\left(\mathcal{I}_{1}<\mathcal{I}_{2}\right)$, and $U_{[0,1]}$ is a uniform random variable on $[0,1]$, independent of $\mathcal{B}$.

We remark that with probability one there is at most one point $x$ such that $\mathcal{L}(t, x)=\mathcal{L}^{*}(t)$ so $F_{t}^{*}$ is actually the favorite site. Note that similar questions about favorite points for $X$ have been studied in the recurrent case $\kappa=0$ by Cheliotis (2008).

One question we may ask here is: what happens in the discrete case (that is, for RWRE), or with a more general Lévy potential?

For RWRE, we expect a very similar behavior because the renewal structures which appear in both cases (RWRE and our diffusion $X$ ) are very similar (see Enriquez et al., 2009a). The main difference comes essentially from the functional $\mathcal{R}_{\kappa}$, which should be replaced by a sum of exponentials of simple random walks conditioned to remain positive (see Enriquez et al., 2009b,a).

For a more general Lévy potential, we have in mind for example a spectrally negative Lévy process (diffusions in such potentials have been studied by Singh, 2008). More work needs to be done, especially for the potential. First, to obtain a specific decomposition of the Lévy's path (similar to what is done for the drifted Brownian motion in Faggionato, 2009), and also to study the more complicated functional $\mathcal{R}_{\kappa}$ which is less known than in the Brownian case. This is a work in preparation by Véchambre (2016).

The rest of the paper is organized as follows.

In Section 2, we recall the results of Faggionato on the path decomposition of the trajectories of $W_{\kappa}$. Also we recall from Andreoletti and Devulder (2015) the construction of specific $h_{t}$-minima which plays an important role in the appearance of independence, under $\mathbb{P}$, on the path of $X$ before time $t$. 
In Section 3, we study the joint process of the hitting times of the $h_{t}$-minima $m_{j}$, $1 \leq j \leq n_{t}$ and of local times at these $m_{j}$. We show that parts of the trajectory of $X$ are not important for our study, that is, we prove that the time spent outside the $h_{t}$-valleys, and the supremum of the local time outside the $h_{t}$-valleys are negligible compared to $t$. We then prove the main result of this section: Proposition 3.5. It shows that the joint process (exit times, local times) can be approximated in probability by $i . i . d$ random variables (which are the $\mathcal{H}_{j}$ and $\ell_{j}$ ). This part makes use of some technical results inspired from Andreoletti and Devulder (2015), they are summarized in Section 6.

In Section 4, we prove Proposition 1.4, and study the continuity of certain functionals of $\left(\mathcal{Y}_{1}, \mathcal{Y}_{2}\right)$ which appear in the expression of the limit law $\mathcal{I}$. This section is independent of the other ones, we essentially prove a basic functional limit theorem and prepare to the application of continuous mapping theorem.

Section 5 is where we make appear the renewal structure in the problem we want to solve. In particular we show how the distribution of the supremum of the local time can be approximated by the distribution of some function of the couple $\left(Y_{1}, Y_{2}\right)^{t}$, the main step being Proposition 5.1.

Section 6 is a reminder of some key results and their extensions extracted from Andreoletti and Devulder (2015). For some of these results, sketch of proofs or complementary proofs are added in order for this paper to be more self-contained.

Finally, Section 7 is a reminder of some estimates on Brownian motion, Bessel processes, and functionals of both of these processes.

1.3. Notation. In this section we introduce typical notation and tools for the study of diffusions in a random potential.

For any process $\left(U(t), \quad t \in \mathbb{R}_{+}\right)$we denote by $\mathcal{L}_{U}$ a bicontinuous version of the local time of $U$ when it exists. Notice that for our main process $X$ we simply write $\mathcal{L}$ for its local time. The inverse of the local time for every $x \in \mathbb{R}$ is denoted by $\sigma_{U}(t, x):=\inf \left\{s>0, \mathcal{L}_{U}(s, x) \geq t\right\}$ and in the same way $\sigma(t, x):=\sigma_{X}(t, x)$. We also denote by $U^{a}$ the process $U$ starting from $a$, and by $P^{a}$ the law of $U^{a}$, with the notation $U=U^{0}$. Now, let us introduce the following functional of $W_{\kappa}$,

$$
A(r):=\int_{0}^{r} e^{W_{\kappa}(x)} \mathrm{d} x, \quad r \in \mathbb{R} .
$$

We recall that since $\kappa>0, A_{\infty}:=\lim _{r \rightarrow+\infty} A(r)<\infty$ a.s. As in Brox (1986), there exists a Brownian motion $(B(s), s \geq 0)$, independent of $W_{\kappa}$, such that $X(t)=A^{-1}\left[B\left(T^{-1}(t)\right)\right]$ for every $t \geq 0$, where

$$
T(r):=\int_{0}^{r} \exp \left\{-2 W_{\kappa}\left[A^{-1}(B(s))\right]\right\} \mathrm{d} s, \quad 0 \leq r<\tau^{B}\left(A_{\infty}\right) .
$$

The local time of the diffusion $X$ at location $x$ and time $t$, simply denoted by $\mathcal{L}(t, x)$, can be written as (see Shi, 1998, eq. (2.5))

$$
\mathcal{L}(t, x)=e^{-W_{\kappa}(x)} \mathcal{L}_{B}\left(T^{-1}(t), A(x)\right), \quad t>0, x \in \mathbb{R} .
$$

With this notation, we recall the following expression of the hitting times of $X$,

$$
H(r)=T\left[\tau^{B}(A(r))\right]=\int_{-\infty}^{r} e^{-W_{\kappa}(u)} \mathcal{L}_{B}\left[\tau^{B}(A(r)), A(u)\right] \mathrm{d} u, \quad r \geq 0 .
$$




\section{Path decomposition and Valleys}

2.1. Path decomposition in the neighborhood of the $h_{t}$-minima $m_{i}$. We first recall some results for $h_{t}$-extrema of $W_{\kappa}$. Let

$$
V^{(i)}(x):=W_{\kappa}(x)-W_{\kappa}\left(m_{i}\right), \quad x \in \mathbb{R}, i \in \mathbb{N}^{*},
$$

which is the potential $W_{\kappa}$ translated so that it is 0 at the local minimum $m_{i}$. We also define

$$
\begin{aligned}
\tau_{i}^{-}(h) & :=\sup \left\{s<m_{i}, V^{(i)}(s)=h\right\}, \quad h>0 \\
\tau_{i}(h) & :=\inf \left\{s>m_{i}, V^{(i)}(s)=h\right\}, \quad h>0 .
\end{aligned}
$$

The following result has been proved by Faggionato (2009) [for (i) and (ii)], and the last fact comes from the strong Markov property (see also Andreoletti and Devulder, 2015, Fact 2.1, and its proof).

Fact 2.1. (path decomposition of $W_{\kappa}$ around the $h_{t}$-minima $m_{i}$ )

(i) The truncated trajectories $\left(V^{(i)}\left(m_{i}-s\right), 0 \leq s \leq m_{i}-\tau_{i}^{-}\left(h_{t}\right)\right),\left(V^{(i)}\left(m_{i}+\right.\right.$ $\left.s), 0 \leq s \leq \tau_{i}\left(h_{t}\right)-m_{i}\right), i \geq 1$ are independent.

(ii) Let $\left(W_{\kappa}^{\uparrow}(s), s \geq 0\right)$ be a process with law $B E S(3, \kappa / 2)$. All the truncated trajectories $\left(V^{(i)}\left(m_{i}-s\right), 0 \leq s \leq m_{i}-\tau_{i}^{-}\left(h_{t}\right)\right)$ for $i \geq 2$ and $\left(V^{(j)}\left(m_{j}+s\right), 0 \leq\right.$ $\left.s \leq \tau_{j}\left(h_{t}\right)-m_{j}\right)$ for $j \geq 1$ are equal in law to $\left(W_{\kappa}^{\uparrow}(s), 0 \leq s \leq \tau^{W_{\kappa}^{\uparrow}}\left(h_{t}\right)\right)$.

(iii) For $i \geq 1$, the truncated trajectory $\left(V^{(i)}\left(s+\tau_{i}\left(h_{t}\right)\right), s \geq 0\right)$ is independent of $\left(W_{\kappa}(s), s \leq \tau_{i}\left(h_{t}\right)\right)$ and is equal in law to $\left(W_{\kappa}^{h_{t}}(s), s \geq 0\right)$, that is, to a $(-\kappa / 2)$-drifted Brownian motion starting from $h_{t}$.

2.2. Definition of $h_{t}$-valleys and of standard $h_{t}$-minima $\tilde{m}_{j}, j \in \mathbb{N}^{*}$.

We are interested in the potential around the $h_{t}$-minima $m_{i}, i \in \mathbb{N}^{*}$, in fact intervals containing at least $\left[\tau_{i}^{-}\left((1+\kappa) h_{t}\right), M_{i}\right]$. However, these valleys could intersect. In order to define valleys which are well separated and i.i.d., we introduce the following notation. This notation is used to define valleys of the potential around some $\tilde{m}_{i}$, which are thanks to Lemma 2.2 equal to the $m_{i}$ for $1 \leq i \leq n_{t}$ with large probability.

Let

$$
h_{t}^{+}:=(1+\kappa+2 \delta) h_{t}
$$

As in Andreoletti and Devulder (2015), we define $\tilde{L}_{0}^{+}:=0, \tilde{m}_{0}:=0$, and recursively for $i \geq 1$ (see Figure 2.1),

$$
\begin{aligned}
\tilde{L}_{i}^{\sharp} & :=\inf \left\{x>\tilde{L}_{i-1}^{+}, W_{\kappa}(x) \leq W_{\kappa}\left(\tilde{L}_{i-1}^{+}\right)-h_{t}^{+}\right\}, \\
\tilde{\tau}_{i}\left(h_{t}\right) & :=\inf \left\{x \geq \tilde{L}_{i}^{\sharp}, W_{\kappa}(x)-\inf _{\left[\tilde{L}_{i}^{\sharp}, x\right]} W_{\kappa} \geq h_{t}\right\}, \\
\tilde{m}_{i} & :=\inf \left\{x \geq \tilde{L}_{i}^{\sharp}, W_{\kappa}(x)=\inf _{\left[\tilde{L}_{i}^{\sharp}, \tilde{\tau}_{i}\left(h_{t}\right)\right]} W_{\kappa}\right\}, \\
\tilde{L}_{i}^{+} & :=\inf \left\{x>\tilde{\tau}_{i}\left(h_{t}\right), W_{\kappa}(x) \leq W_{\kappa}\left(\tilde{\tau}_{i}\left(h_{t}\right)\right)-h_{t}-h_{t}^{+}\right\} .
\end{aligned}
$$

We also introduce the following random variables for $i \in \mathbb{N}^{*}$ : 


$$
\begin{aligned}
\tilde{M}_{i} & :=\inf \left\{s>\tilde{m}_{i}, W_{\kappa}(s)=\max _{\tilde{m}_{i} \leq u \leq \tilde{L}_{i}^{+}} W_{\kappa}(u)\right\}, \\
\tilde{L}_{i}^{*} & :=\inf \left\{x>\tilde{\tau}_{i}\left(h_{t}\right), W_{\kappa}(x)-W_{\kappa}\left(\tilde{m}_{i}\right)=3 h_{t} / 4\right\}, \\
\tilde{L}_{i} & :=\inf \left\{x>\tilde{\tau}_{i}\left(h_{t}\right), W_{\kappa}(x)-W_{\kappa}\left(\tilde{m}_{i}\right)=h_{t} / 2\right\}, \\
\tilde{\tau}_{i}(h) & :=\inf \left\{s>\tilde{m}_{i}, W_{\kappa}(x)-W_{\kappa}\left(\tilde{m}_{i}\right)=h\right\}, \quad h>0, \\
\tilde{\tau}_{i}^{-}(h) & :=\sup \left\{s<\tilde{m}_{i}, W_{\kappa}(x)-W_{\kappa}\left(\tilde{m}_{i}\right)=h\right\}, \quad h>0, \\
\tilde{L}_{i}^{-} & :=\tilde{\tau}_{i}^{-}\left(h_{t}^{+}\right) .
\end{aligned}
$$

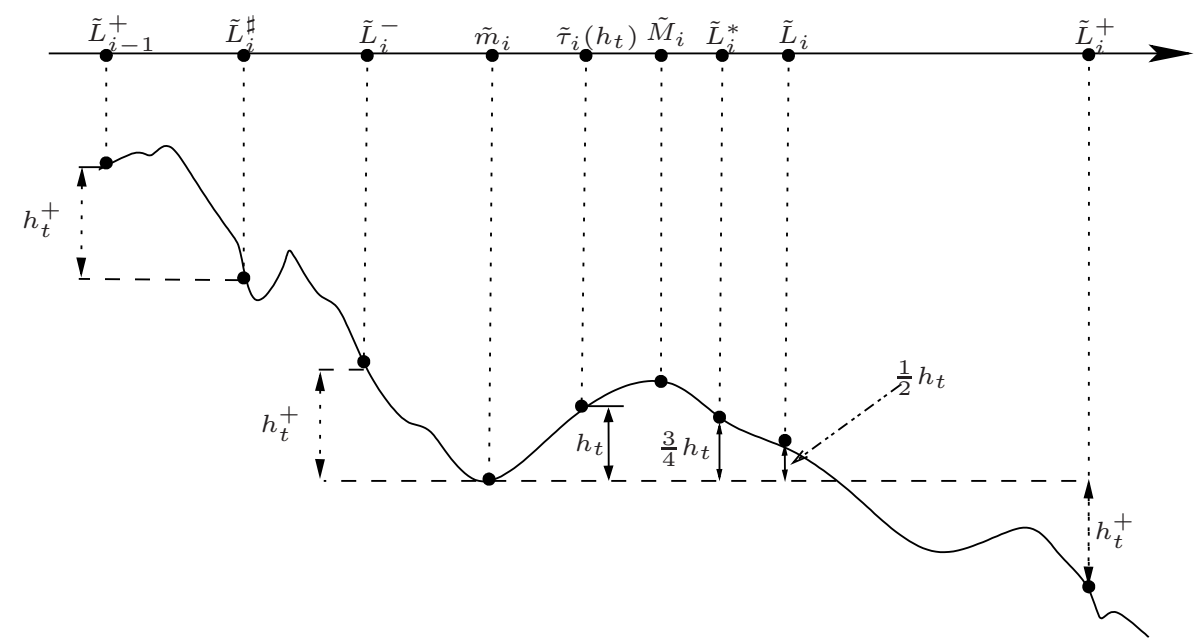

Figure 2.1. Schema of the potential between $\tilde{L}_{i-1}^{+}$and $\tilde{L}_{i}^{+}$, in the case $\tilde{L}_{i}^{\sharp}<\tilde{L}_{i}^{-}$.

We stress that these random variables depend on $t$, which we do not write as a subscript to simplify the notation. Notice also that $\tilde{\tau}_{i}\left(h_{t}\right)$ is the same in definitions (2.3) and (2.5) with $h=h_{t}$. Moreover by continuity of $W_{\kappa}, W_{\kappa}\left(\tilde{\tau}_{i}\left(h_{t}\right)\right)=W_{\kappa}\left(\tilde{m}_{i}\right)+$ $h_{t}$. Thus, the $\tilde{m}_{i}, i \in \mathbb{N}^{*}$, are $h_{t}$-minima, since $W_{\kappa}\left(\tilde{m}_{i}\right)=\inf _{\left[\tilde{L}_{i-1}^{+}, \tilde{\tau}_{i}\left(h_{t}\right)\right]} W_{\kappa}$, $W_{\kappa}\left(\tilde{\tau}_{i}\left(h_{t}\right)\right)=W_{\kappa}\left(\tilde{m}_{i}\right)+h_{t}$ and $W_{\kappa}\left(\tilde{L}_{i-1}^{+}\right) \geq W_{\kappa}\left(\tilde{m}_{i}\right)+h_{t}$. In addition,

$$
\begin{gathered}
\tilde{L}_{i-1}^{+}<\tilde{L}_{i}^{\sharp} \leq \tilde{m}_{i}<\tilde{\tau}_{i}\left(h_{t}\right)<\tilde{L}_{i}^{*}<\tilde{L}_{i}<\tilde{L}_{i}^{+}, \quad i \in \mathbb{N}^{*}, \\
\tilde{L}_{i-1}^{+} \leq \tilde{L}_{i}^{-}<\tilde{m}_{i}<\tilde{\tau}_{i}\left(h_{t}\right)<\tilde{M}_{i}<\tilde{L}_{i}^{+}, \quad i \in \mathbb{N}^{*} .
\end{gathered}
$$

Also by induction, the random variables $\tilde{L}_{i}^{\sharp}, \tilde{\tau}_{i}\left(h_{t}\right)$ and $\tilde{L}_{i}^{+}, i \in \mathbb{N}^{*}$ are stopping times for the natural filtration of $\left(W_{\kappa}(x), x \geq 0\right)$, and so $\tilde{L}_{i}, \tilde{L}_{i}^{*}, i \in \mathbb{N}^{*}$, are also stopping times. Moreover by induction,

$$
\begin{array}{r}
W_{\kappa}\left(\tilde{L}_{i}^{\sharp}\right)=\inf _{\left[0, \tilde{L}_{i}^{\sharp}\right]} W_{\kappa}, \quad W_{\kappa}\left(\tilde{m}_{i}\right)=\inf _{\left[0, \tilde{\tau}_{i}\left(h_{t}\right)\right]} W_{\kappa}, \\
W_{\kappa}\left(\tilde{L}_{i}^{+}\right)=\inf _{\left[0, \tilde{L}_{i}^{+}\right]} W_{\kappa}=W_{\kappa}\left(\tilde{m}_{i}\right)-h_{t}^{+},
\end{array}
$$

for $i \in \mathbb{N}^{*}$. We also introduce the analogue of $V^{(i)}$ for $\tilde{m}_{i}$ as follows:

$$
\tilde{V}^{(i)}(x):=W_{\kappa}(x)-W_{\kappa}\left(\tilde{m}_{i}\right), \quad x \in \mathbb{R}, i \in \mathbb{N}^{*} .
$$




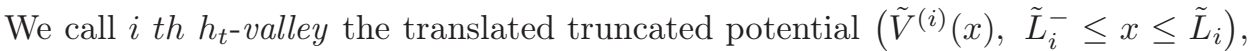
for $i \geq 1$.

The following lemma states that, with a very large probability, the first $n_{t}+1$ positive $h_{t}$-minima $m_{i}, 1 \leq i \leq n_{t}+1$, coincide with the random variables $\tilde{m}_{i}$, $1 \leq i \leq n_{t}+1$. We introduce the corresponding event $\mathcal{V}_{t}:=\cap_{i=1}^{n_{t}+1}\left\{m_{i}=\tilde{m}_{i}\right\}$.

Lemma 2.2. Assume $0<\delta<1$. There exists a constant $C_{1}>0$ such that for $t$ large enough,

$$
P\left(\overline{\mathcal{V}}_{t}\right) \leq C_{1} n_{t} e^{-\kappa h_{t} / 2}=e^{[-\kappa / 2+o(1)] h_{t}} .
$$

Moreover, the sequence $\left(\left(\tilde{V}^{(i)}\left(x+\tilde{L}_{i-1}^{+}\right), 0 \leq x \leq \tilde{L}_{i}^{+}-\tilde{L}_{i-1}^{+}\right), i \geq 1\right)$, is i.i.d.

Proof: This lemma is proved in Andreoletti and Devulder (2015): Lemma 2.3.

The following remark is used several times in the rest of the paper.

Remark 2.3. On $\mathcal{V}_{t}$, we have for every $1 \leq i \leq n_{t}, m_{i}=\tilde{m}_{i}$, and as a consequence, $\tilde{V}^{(i)}(x)=V^{(i)}(x), x \in \mathbb{R}, \tau_{i}^{-}(h)=\tilde{\tau}_{i}^{-}(h)$ and $\tau_{i}(h)=\tilde{\tau}_{i}(h)$ for $h>0$. Moreover, $\tilde{M}_{i}=M_{i}$. Indeed, $\tilde{M}_{i}$ is an $h_{t}$-maximum for $W_{\kappa}$, which belongs to $\left[\tilde{m}_{i}, \tilde{m}_{i+1}\right]=$ $\left[m_{i}, m_{i+1}\right]$ on $\mathcal{V}_{t}$, and there is exactly one $h_{t}$-maximum in this interval since the $h_{t}$-maxima and minima alternate, which we defined as $M_{i}$, so $\tilde{M}_{i}=M_{i}$. So in the following, on $\mathcal{V}_{t}$, we can write these random variables with or without tilde.

\section{Contributions for hitting and local times}

3.1. Negligible parts for hitting times.

In the following lemma we recall results of Andreoletti and Devulder (2015) which say, roughly speaking, that the time spent by the diffusion $X$ outside the $h_{t}$-valleys is negligible compared to the amount of time spent by $X$ inside the $h_{t}$-valleys. This lemma also gives an upper bound for the number of $h_{t}$-valleys visited before time $t$. Finally, it tells us that with large probability, up to time $t$, after first hitting the bottom $\tilde{m}_{j}$ of each $h_{t}$-valley $\left[\tilde{L}_{j}^{-}, \tilde{L}_{j}\right], X$ leaves this $h_{t}$-valley on its right, that is on $\tilde{L}_{j}$, and that $X$ never backtracks in a previously visited $h_{t}$-valley. We define $H_{x \rightarrow y}:=\inf \{s>H(x), X(s)=y\}-H(x)$ for any $x \geq 0$ and $y \geq 0$, which is equal to $H(y)-H(x)$ if $x<y$. Let

$$
\begin{aligned}
& U_{0}:=0, \quad U_{i}:=H\left(\tilde{L}_{i}\right)-H\left(\tilde{m}_{i}\right)=H_{\tilde{m}_{i} \rightarrow \tilde{L}_{i}}, \quad i \geq 1, \\
& \mathcal{B}_{1}(m):=\bigcap_{k=1}^{m}\left\{0 \leq H\left(\tilde{m}_{k}\right)-\sum_{i=1}^{k-1} U_{i}<\tilde{v}_{t}\right\}, \quad m \geq 1,
\end{aligned}
$$

where $\tilde{v}_{t}:=2 t / \log h_{t}$ and $\sum_{i=1}^{0} U_{i}=0$ by convention. Finally, we introduce

$$
\mathcal{B}_{2}(m):=\bigcap_{j=1}^{m}\left\{H_{\tilde{m}_{j} \rightarrow \tilde{L}_{j}}<H_{\tilde{m}_{j} \rightarrow \tilde{L}_{j}^{-}}, H_{\tilde{L}_{j} \rightarrow \tilde{m}_{j+1}}<H_{\tilde{L}_{j} \rightarrow \tilde{L}_{j}^{*}}\right\}, \quad m \geq 1 .
$$

Lemma 3.1. For any $\delta>0$ small enough, we have for all large $t$,

$$
\mathbb{P}\left[H\left(\tilde{m}_{1}\right) \leq \tilde{v}_{t}\right] \geq \mathbb{P}\left[\mathcal{B}_{1}\left(n_{t}\right)\right] \geq 1-C_{2} v_{t},
$$


with $v_{t}:=n_{t} \cdot\left(\log h_{t}\right) e^{-\phi(t)}=o(1)$ and $C_{2}>0$. Moreover, there exists $C_{3}>0$ such that for large $t$,

$$
\begin{aligned}
\mathbb{P}\left(\mathcal{B}_{2}\left(n_{t}\right)\right) & \geq 1-C_{3} n_{t} e^{-\delta \kappa h_{t}} \\
\mathbb{P}\left(N_{t}<n_{t}\right) & \geq 1-e^{-\phi(t)}
\end{aligned}
$$

Proof: The first statement is Lemma 3.7 in Andreoletti and Devulder (2015). The second one follows directly from Lemmata 3.2 and 3.3 in Andreoletti and Devulder (2015). For the proof of (3.3) see Lemma 6.1.

\subsection{Negligible parts for local times.}

We now provide estimates for the local time of $X$ at time $t$. We first prove that the local time of $X$ outside the first $n_{t} h_{t}$-valleys is negligible compared to $t$. Second, we prove that for every $1 \leq j \leq n_{t}$ the local time of $X$ inside the $h_{t}$-valley $\left[\tilde{L}_{j}^{-}, \tilde{L}_{j}\right]$ but outside a small neighborhood of $\tilde{m}_{j}$ is also negligible compared to $t$.

3.2.1. Supremum of the local time outside the valleys.

The aim of this subsection is to prove that at time $t$, the maximum of the local time outside the $h_{t}$-valleys is negligible compared to $t$. More precisely, let $f(t):=$ $t e^{[\kappa(1+3 \delta)-1] \phi(t)}$ and, for $m \geq 1$,

$$
\begin{aligned}
& \mathcal{B}_{3}^{1}(m):=\left\{\sup _{x \in\left[0, \tilde{m}_{1}\right]} \mathcal{L}\left(H\left(\tilde{m}_{1}\right), x\right) \leq f(t)\right\} \\
& \cap \bigcap_{j=1}^{m-1}\left\{\sup _{x \in\left[\tilde{L}_{j}, \tilde{m}_{j+1}\right]} \mathcal{L}\left(H\left(\tilde{m}_{j+1}\right), x\right) \leq f(t)\right\}, \\
& \mathcal{B}_{3}^{2}(m):=\bigcap_{j=1}^{m-1}\left\{\sup _{x \leq \tilde{L}_{j}}\left(\mathcal{L}\left(H\left(\tilde{m}_{j+1}\right), x\right)-\mathcal{L}(H(\tilde{L} j), x)\right) \leq f(t)\right\}, \\
& \mathcal{B}_{3}(m):=\mathcal{B}_{3}^{1}(m) \cap \mathcal{B}_{3}^{2}(m) .
\end{aligned}
$$

This section is devoted to the proof of the following lemma.

Lemma 3.2. Assume that $\delta$ is small enough such that $\kappa(1+3 \delta)<1$. There exists $C_{5}>0$ such that for any large $t$

$$
\mathbb{P}\left(\mathcal{B}_{3}\left(n_{t}\right)\right) \geq 1-C_{5} w_{t},
$$

with $w_{t}:=e^{-\kappa \delta \phi(t)}$.

Its proof is based on Lemma 3.3 below, for which we introduce the following notation, depending only on the potential $W_{\kappa}$ :

$$
\begin{aligned}
\tau_{1}^{*}(h) & :=\inf \left\{u \geq 0, W_{\kappa}(u)-\inf _{[0, u]} W_{\kappa} \geq h\right\}, \quad h>0, \\
m_{1}^{*}(h) & :=\inf \left\{y \geq 0, W_{\kappa}(y)=\inf _{\left[0, \tau_{1}^{*}(h)\right]} W_{\kappa}\right\}, \quad h>0 .
\end{aligned}
$$

Throughout the paper, $C_{+}$(resp. $\left.c_{-}\right)$denotes a positive constant that may grow (resp. decrease) from line to line.

Lemma 3.3. Assume that $\kappa(1+3 \delta)<1$. For large $t$,

$$
\mathbb{P}\left(\sup _{x \in\left[0, m_{1}^{*}\left(h_{t}\right)\right]} \mathcal{L}\left[H\left(\tau_{1}^{*}\left(h_{t}\right)\right), x\right]>t e^{[\kappa(1+3 \delta)-1] \phi(t)}\right) \leq \frac{C_{+}}{n_{t} e^{\kappa \delta \phi(t)}} .
$$


Proof of Lemma 3.3: Thanks to (1.12) and (1.13) there exists a Brownian motion $(B(s), s \geq 0)$, independent of $W_{\kappa}$, such that

$$
\mathcal{L}\left[H\left(\tau_{1}^{*}\left(h_{t}\right)\right), x\right]=e^{-W_{\kappa}(x)} \mathcal{L}_{B}\left[\tau^{B}\left(A\left(\tau_{1}^{*}\left(h_{t}\right)\right)\right), A(x)\right], \quad x \in \mathbb{R} .
$$

By the first Ray-Knight theorem (see e.g. Revuz and Yor, 1999, chap. XI), for every $\alpha>0$, there exists a Bessel processes $Q_{2}$ of dimension 2 starting from 0 , such that $\mathcal{L}_{B}\left(\tau^{B}(\alpha), x\right)$ is equal to $Q_{2}^{2}(\alpha-x)$ for every $x \in[0, \alpha]$. Consequently, using (3.5) and the independence of $B$ and $W_{\kappa}$, there exists a 2-dimensional Bessel process $Q_{2}$ such that

$$
\mathcal{L}\left[H\left(\tau_{1}^{*}\left(h_{t}\right)\right), x\right]=e^{-W_{\kappa}(x)} Q_{2}^{2}\left[A\left(\tau_{1}^{*}\left(h_{t}\right)\right)-A(x)\right] \quad 0 \leq x \leq \tau_{1}^{*}\left(h_{t}\right) .
$$

In order to evaluate this quantity, the idea is to say that loosely speaking, $Q_{2}^{2}$ grows almost linearly. More formally, we consider the functions $k(t):=e^{2 \kappa^{-1} \phi(t)}$, $a(t):=4 \phi(t)$ and $b(t):=6 \kappa^{-1} \phi(t) e^{\kappa h_{t}}$, and define the following events

$$
\begin{aligned}
& \mathcal{A}_{0}:=\left\{A_{\infty}:=\int_{0}^{+\infty} e^{W_{\kappa}(u)} \mathrm{d} u \leq k(t)\right\}, \\
& \mathcal{A}_{1}:=\left\{\forall u \in(0, k(t)], Q_{2}^{2}(u) \leq 2 e u[a(t)+4 \log \log [e k(t) / u]]\right\}, \\
& \mathcal{A}_{2}:=\left\{\inf _{\left[0, \tau_{1}^{*}\left(h_{t}\right)\right]} W_{\kappa} \geq-b(t)\right\} .
\end{aligned}
$$

We know that $P\left(A_{\infty} \geq y\right) \leq C_{+} y^{-\kappa}$ for $y>0$ since $2 / A_{\infty}$ is a gamma variable of parameter $(\kappa, 1)$ (see Dufresne, 2000, or Borodin and Salminen, 2002 IV.48 p. 78), having a density equal to $e^{-x} x^{\kappa-1} \mathbb{1}_{\mathbb{R}_{+}}(x) / \Gamma(\kappa)$, so $P\left(\overline{\mathcal{A}_{0}}\right) \leq C_{+} k(t)^{-\kappa}=$ $C_{+} e^{-2 \phi(t)}$. Moreover, $\mathbb{P}\left(\overline{\mathcal{A}}_{1}\right) \leq C_{+} \exp [-a(t) / 2]=C_{+} e^{-2 \phi(t)}$ by Lemma 7.5. Also we know that $-\inf _{\left[0, \tau_{1}^{*}(h)\right]} W_{\kappa}$, denoted by $-\beta$ in Faggionato (2009, eq. (2.2)), is exponentially distributed with mean $2 \kappa^{-1} \sinh (\kappa h / 2) e^{\kappa h / 2}$ (Faggionato, 2009, eq. (2.4)). So for large $t$,

$$
\begin{aligned}
P\left(\overline{\mathcal{A}}_{2}\right) & =P\left[-\inf _{\left[0, \tau_{1}^{*}\left(h_{t}\right)\right]} W_{\kappa}>b(t)\right] \\
& =\exp \left[-b(t) \kappa /\left(2 \sinh \left(\kappa h_{t} / 2\right) e^{\kappa h_{t} / 2}\right)\right] \\
& \leq e^{-2 \phi(t)}
\end{aligned}
$$

Now, assume we are on $\mathcal{A}_{0} \cap \mathcal{A}_{1} \cap \mathcal{A}_{2}$. Due to (3.6), we have for every $0 \leq x<\tau_{1}^{*}\left(h_{t}\right)$, since $0<A\left(\tau_{1}^{*}\left(h_{t}\right)\right)-A(x) \leq A_{\infty} \leq k(t)$,

$$
\begin{aligned}
& \mathcal{L}\left[H\left(\tau_{1}^{*}\left(h_{t}\right)\right), x\right] \\
& \leq e^{-W_{\kappa}(x)} 2 e\left[A\left(\tau_{1}^{*}\left(h_{t}\right)\right)-A(x)\right]\left\{a(t)+4 \log \log \left[e k(t) /\left[A\left(\tau_{1}^{*}\left(h_{t}\right)\right)-A(x)\right]\right]\right\} .
\end{aligned}
$$

We now introduce

$$
f_{i}:=\inf \left\{u \geq 0, W_{\kappa}(u) \leq-i\right\}=\tau^{W_{\kappa}}(-i), \quad i \in \mathbb{N},
$$

and let $0 \leq x<\tau_{1}^{*}\left(h_{t}\right)$. There exists $i \in \mathbb{N}$ such that $f_{i} \leq x<f_{i+1}$. Moreover, we are on $\mathcal{A}_{2}$, so $i \leq b(t)$. Furthermore, $x<f_{i+1}$, so $W_{\kappa}(x) \geq-(i+1)$ and then $e^{-W_{\kappa}(x)} \leq e^{i+1}=e^{-W_{\kappa}\left(f_{i}\right)+1}$. All this leads to

$$
\begin{aligned}
e^{-W_{\kappa}(x)}\left[A\left(\tau_{1}^{*}\left(h_{t}\right)\right)-A(x)\right] & =e^{-W_{\kappa}(x)} \int_{x}^{\tau_{1}^{*}\left(h_{t}\right)} e^{W_{\kappa}(u)} \mathrm{d} u \\
& \leq e \int_{f_{i}}^{\tau_{1}^{*}\left(h_{t}\right)} e^{W_{\kappa}(u)-W_{\kappa}\left(f_{i}\right)} \mathrm{d} u .
\end{aligned}
$$


To bound this, we introduce the event

$$
\mathcal{A}_{3}:=\bigcap_{i=0}^{\lfloor b(t)\rfloor}\left\{\int_{f_{i}}^{\tau_{1}^{*}\left(h_{t}\right)} e^{W_{\kappa}(u)-W_{\kappa}\left(f_{i}\right)} \mathrm{d} u \leq e^{(1-\kappa) h_{t}} b(t) n_{t} e^{\kappa \delta \phi(t)}\right\} .
$$

We now consider $\tau_{1}^{*}\left(u, h_{t}\right):=\inf \left\{y \geq u, W_{\kappa}(y)-\inf _{[u, y]} W_{\kappa} \geq h_{t}\right\} \geq \tau_{1}^{*}\left(h_{t}\right)$ for $u \geq 0$. We have

$$
E\left(\int_{f_{i}}^{\tau_{1}^{*}\left(h_{t}\right)} e^{W_{\kappa}(u)-W_{\kappa}\left(f_{i}\right)} \mathrm{d} u\right) \leq E\left(\int_{f_{i}}^{\tau_{1}^{*}\left(f_{i}, h_{t}\right)} e^{W_{\kappa}(u)-W_{\kappa}\left(f_{i}\right)} \mathrm{d} u\right)=\beta_{0}\left(h_{t}\right),
$$

by the strong Markov property applied at stopping time $f_{i}$, where we define $\beta_{0}(h):=$ $E\left(\int_{0}^{\tau_{1}^{*}(h)} e^{W_{\kappa}(u)} \mathrm{d} u\right)$. By $(6.15), \beta_{0}(h) \leq C_{+} e^{(1-\kappa) h}$ for large $h$. Hence for large $t$ by Markov inequality,

$$
\begin{aligned}
P\left(\overline{\mathcal{A}}_{3}\right) & \leq \sum_{i=0}^{\lfloor b(t)\rfloor} P\left(\int_{f_{i}}^{\tau_{1}^{*}\left(h_{t}\right)} e^{W_{\kappa}(u)-W_{\kappa}\left(f_{i}\right)} \mathrm{d} u>e^{(1-\kappa) h_{t}} b(t) n_{t} e^{\kappa \delta \phi(t)}\right) \\
& \leq \frac{[b(t)+1] \beta_{0}\left(h_{t}\right)}{e^{(1-\kappa) h_{t}} b(t) n_{t} e^{\kappa \delta \phi(t)}} \leq \frac{C_{+}}{n_{t} e^{\kappa \delta \phi(t)}} .
\end{aligned}
$$

Now, on $\cap_{j=0}^{3} \mathcal{A}_{j}$, (3.7) and (3.8) lead to

$$
\begin{aligned}
& \mathcal{L}\left[H\left(\tau_{1}^{*}\left(h_{t}\right)\right), x\right] \\
\leq & 2 e^{2+(1-\kappa) h_{t}} b(t) n_{t} e^{\kappa \delta \phi(t)}\left\{a(t)+4 \log \log \left[e k(t) /\left[A\left(\tau_{1}^{*}\left(h_{t}\right)\right)-A(x)\right]\right]\right\} .
\end{aligned}
$$

We now consider only $0 \leq x \leq m_{1}^{*}\left(h_{t}\right)$. By definition of $\mathcal{A}_{2}, \inf _{\left[0, \tau_{1}^{*}\left(h_{t}\right)\right]} W_{\kappa} \geq-b(t)$, such that

$$
\begin{aligned}
A\left(\tau_{1}^{*}\left(h_{t}\right)\right)-A(x) & =\int_{x}^{\tau_{1}^{*}\left(h_{t}\right)} e^{W_{\kappa}(u)} \mathrm{d} u \\
& \geq \int_{m_{1}^{*}\left(h_{t}\right)}^{\tau_{1}^{*}\left(h_{t}\right)} e^{W_{\kappa}(u)} \mathrm{d} u \\
& \geq e^{-b(t)}\left[\tau_{1}^{*}\left(h_{t}\right)-m_{1}^{*}\left(h_{t}\right)\right] \\
& \geq e^{-b(t)}
\end{aligned}
$$

on the event $\cap_{i=0}^{4} \mathcal{A}_{i}$ with $\mathcal{A}_{4}:=\left\{\tau_{1}^{*}\left(h_{t}\right)-m_{1}^{*}\left(h_{t}\right) \geq 1\right\}$. Since $m_{1}=m_{1}^{*}\left(h_{t}\right)$ and $\tau_{1}\left(h_{t}\right)=\tau_{1}^{*}\left(h_{t}\right)$ on $\left\{M_{0} \leq 0\right\}$ by definition of $h_{t}$-extrema, we have

$$
\begin{aligned}
P\left(\overline{\mathcal{A}}_{4}\right) & \leq P\left(0<M_{0}<m_{1}\right)+P\left[\tau_{1}\left(h_{t}\right)-m_{1}<1\right] \\
& \leq C_{+} h_{t} e^{-\kappa h_{t}}+P\left[\tau^{W_{\kappa}^{\uparrow}}\left(h_{t}\right)-\tau^{W_{\kappa}^{\uparrow}}\left(h_{t} / 2\right)<1\right] \\
& \leq C_{+} h_{t} e^{-\kappa h_{t}}+C_{+} \exp \left[-\left(c_{-}\right) h_{t}^{2}\right]
\end{aligned}
$$

due to Andreoletti and Devulder (2015, eq. (2.8)), coming from Faggionato (2009), Fact 2.1 (ii) and (7.4).

Now, we have $e k(t) /\left[A\left(\tau_{1}^{*}\left(h_{t}\right)\right)-A(x)\right] \leq e k(t) e^{b(t)}$ on $\cap_{i=0}^{4} \mathcal{A}_{i}$, and then, on this event, (3.9) leads to

$$
\begin{aligned}
\mathcal{L}\left[H\left(\tau_{1}^{*}\left(h_{t}\right)\right), x\right] & \leq 2 e^{2+(1-\kappa) h_{t}} b(t) n_{t} e^{\kappa \delta \phi(t)}\left\{a(t)+4 \log \log \left[e k(t) e^{b(t)}\right]\right\} . \\
& \leq C_{+} t \phi(t) e^{[\kappa(1+\delta)-1] \phi(t)} e^{\kappa \delta \phi(t)} h_{t},
\end{aligned}
$$


since $\phi(t)=o(\log t), h_{t}=\log t-\phi(t)$ and $n_{t}=\left\lfloor e^{\kappa(1+\delta) \phi(t)}\right\rfloor$. We notice that for large $t, C_{+} \phi(t) h_{t} \leq e^{\kappa \delta \phi(t)}$ since $\log \log t=o(\phi(t))$. Hence, for large $t$,

$$
\mathcal{L}\left[H\left(\tau_{1}^{*}\left(h_{t}\right)\right), x\right] \leq t e^{[\kappa(1+3 \delta)-1] \phi(t)},
$$

on $\cap_{i=0}^{4} \mathcal{A}_{i}$ for every $0 \leq x \leq m_{1}^{*}\left(h_{t}\right)$. This gives for large $t$,

$\mathbb{P}\left(\sup _{x \in\left[0, m_{1}^{*}\left(h_{t}\right)\right]} \mathcal{L}\left[H\left(\tau_{1}^{*}\left(h_{t}\right)\right), x\right] \leq t e^{[\kappa(1+3 \delta)-1] \phi(t)}\right) \geq \mathbb{P}\left(\cap_{i=0}^{4} \mathcal{A}_{i}\right) \geq 1-\frac{C_{+}}{n_{t} e^{\kappa \delta \phi(t)}}$,

due to the previous bounds for $\mathbb{P}\left(\mathcal{A}_{i}\right), 0 \leq i \leq 4$. This proves the lemma.

With the help of the previous lemma, we can now prove Lemma 3.2.

Proof of Lemma 3.2: The method is to do a coupling, similarly as in the proof of Lemma 3.7 of Andreoletti and Devulder (2015). Recall the definition of $\tilde{L}_{i}^{*}<\tilde{L}_{i}<$ $\tilde{L}_{i+1}^{\sharp}$ just above (2.5). Also, let

$$
\begin{aligned}
\tilde{\tau}_{i+1}^{*}\left(h_{t}\right) & :=\inf \left\{u \geq \tilde{L}_{i}^{*}, W_{\kappa}(u)-\inf _{\left[\tilde{L}_{i}^{*}, u\right]} W_{\kappa} \geq h_{t}\right\} \leq \tilde{\tau}_{i+1}\left(h_{t}\right), \quad i \geq 1, \\
\tilde{m}_{i+1}^{*}\left(h_{t}\right) & :=\inf \left\{u \geq \tilde{L}_{i}^{*}, W_{\kappa}(u)=\inf _{\left[\tilde{L}_{i}^{*}, \tilde{\tau}_{i+1}^{*}\left(h_{t}\right)\right]} W_{\kappa}\right\}, \quad i \geq 1, \\
\mathcal{A}_{5} & :=\cap_{i=1}^{n_{t}-1}\left\{\tilde{\tau}_{i+1}^{*}\left(h_{t}\right)=\tilde{\tau}_{i+1}\left(h_{t}\right)\right\}, \\
X_{i}(u) & :=X\left(u+H\left(\tilde{L}_{i}\right)\right), \quad X_{i}^{*}(u):=X\left(u+H\left(\tilde{L}_{i}^{*}\right)\right), \quad u \geq 0, i \geq 1 .
\end{aligned}
$$

Let $i \geq 1$. By the strong Markov property, $X_{i}$ and $X_{i}^{*}$ are diffusions in the potential $W_{\kappa}$, starting respectively from $\tilde{L}_{i}$ and $\tilde{L}_{i}^{*}$. We denote respectively by $\mathcal{L}_{X_{i}}, \mathcal{L}_{X_{i}^{*}}$, $H_{X_{i}}$ and $H_{X_{i}^{*}}$ the local times and hitting times of $X_{i}$ and $X_{i}^{*}$. We have for every $x \geq \tilde{L}_{i}^{*}$,

$$
\begin{aligned}
\mathcal{L}\left(H\left(\tilde{m}_{i+1}\right), x\right)-\mathcal{L}\left(H\left(\tilde{L}_{i}\right), x\right) & \leq \mathcal{L}\left(H\left(\tilde{m}_{i+1}\right), x\right)-\mathcal{L}\left(H\left(\tilde{L}_{i}^{*}\right), x\right) \\
& =\mathcal{L}_{X_{i}^{*}}\left(H_{X_{i}^{*}}\left(\tilde{m}_{i+1}\right), x\right)
\end{aligned}
$$

Consequently, on $\mathcal{A}_{5} \cap \mathcal{A}_{6}$ with $\mathcal{A}_{6}:=\cap_{j=1}^{n_{t}-1}\left\{H_{X_{j}}\left(\tilde{m}_{j+1}\right)<H_{X_{j}}\left(\tilde{L}_{j}^{*}\right)\right\}$, for $1 \leq i \leq$ $n_{t}-1$,

$$
\begin{aligned}
& \sup _{x \in \mathbb{R}}\left(\mathcal{L}\left(H\left(\tilde{m}_{i+1}\right), x\right)-\mathcal{L}\left(H\left(\tilde{L}_{i}\right), x\right)\right) \\
= & \sup _{\tilde{L}_{i}^{*} \leq x \leq \tilde{m}_{i+1}}\left(\mathcal{L}\left(H\left(\tilde{m}_{i+1}\right), x\right)-\mathcal{L}\left(H\left(\tilde{L}_{i}\right), x\right)\right) \\
\leq & \sup _{\tilde{L}_{i}^{*} \leq x \leq \tilde{m}_{i+1}} \mathcal{L}_{X_{i}^{*}}\left(H_{X_{i}^{*}}\left(\tilde{m}_{i+1}\right), x\right) \\
\leq & \sup _{\tilde{L}_{i}^{*} \leq x \leq \tilde{m}_{i+1}^{*}} \mathcal{L}_{X_{i}^{*}}\left(H_{X_{i}^{*}}\left(\tilde{\tau}_{i+1}^{*}\left(h_{t}\right)\right), x\right),
\end{aligned}
$$

since $\tilde{m}_{i+1}^{*}=\tilde{m}_{i+1} \leq \tilde{\tau}_{i+1}\left(h_{t}\right)=\tilde{\tau}_{i+1}^{*}\left(h_{t}\right)$ on $\mathcal{A}_{5}$. Now, notice that the right hand side of (3.11) is the supremum of the local times of $X_{i}^{*}-\tilde{L}_{i}^{*}$, up to its first hitting time of $\tilde{\tau}_{i+1}^{*}\left(h_{t}\right)-\tilde{L}_{i}^{*}$, over all locations in $\left[0, \tilde{m}_{i+1}^{*}-\tilde{L}_{i}^{*}\right]$. Since $X_{i}^{*}-$ $\tilde{L}_{i}^{*}$ is a diffusion in the potential $\left(W_{\kappa}\left(\tilde{L}_{i}^{*}+x\right)-W_{\kappa}\left(\tilde{L}_{i}^{*}\right), x \in \mathbb{R}\right)$, which has on $[0,+\infty)$ the same law as $\left(W_{\kappa}(x), x \geq 0\right)$ because $\tilde{L}_{i}^{*}$ is a stopping time for $W_{\kappa}$, the 
right hand side of (3.11) has the same law, under the annealed probability $\mathbb{P}$, as $\sup _{x \in\left[0, m_{1}^{*}\left(h_{t}\right)\right]} \mathcal{L}\left[H\left(\tau_{1}^{*}\left(h_{t}\right)\right), x\right]$. Consequently,

$$
\begin{aligned}
& \mathbb{P}\left(\bigcup_{i=1}^{n_{t}-1}\left\{\sup _{x \in \mathbb{R}}\left(\mathcal{L}\left(H\left(\tilde{m}_{i+1}\right), x\right)-\mathcal{L}\left(H\left(\tilde{L}_{i}\right), x\right)\right)>t e^{[\kappa(1+3 \delta)-1] \phi(t)}\right\}\right) \\
\leq & n_{t}\left[\mathbb{P}\left(\sup _{x \in\left[0, m_{1}^{*}\left(h_{t}\right)\right]} \mathcal{L}\left[H\left(\tau_{1}^{*}\left(h_{t}\right)\right), x\right]>t e^{[\kappa(1+3 \delta)-1] \phi(t)}\right)+\mathbb{P}\left(\overline{\mathcal{A}}_{5}\right)+\mathbb{P}\left(\overline{\mathcal{A}}_{6}\right)\right] \\
\leq & C_{+} e^{-\kappa \delta \phi(t)}
\end{aligned}
$$

by Lemma 3.3 , since $\mathbb{P}\left(\overline{\mathcal{A}}_{5}\right) \leq C_{+} n_{t} h_{t} e^{-\kappa h_{t}}$ by $(6.9), \mathbb{P}\left(\overline{\mathcal{A}}_{6}\right) \leq \mathbb{P}\left(\overline{\mathcal{B}_{2}}\left(n_{t}\right)\right) \leq$ $C_{3} n_{t} e^{-\delta \kappa h_{t}}$ by (3.2) and since $\phi(t)=o(\log t)$. Notice that, as before, $\tilde{m}_{1}=m_{1}=$ $m_{1}^{*}\left(h_{t}\right)$ on $\mathcal{V}_{t} \cap\left\{M_{0} \leq 0\right\}$. Finally,

$$
\begin{aligned}
\mathbb{P}\left(\sup _{x \in\left[0, \tilde{m}_{1}\right]} \mathcal{L}\left(H\left(\tilde{m}_{1}\right), x\right)>t e^{[\kappa(1+3 \delta)-1] \phi(t)}\right) & \leq \frac{C_{+}}{e^{\kappa \delta \phi(t)}}+P\left(\overline{\mathcal{V}_{t}}\right)+P\left(0<M_{0}<m_{1}\right) \\
& \leq \frac{C_{+}}{e^{\kappa \delta \phi(t)}}
\end{aligned}
$$

also by Lemma 3.3, Lemma 2.2, and since $P\left(0<M_{0}<m_{1}\right) \leq C_{+} h_{t} e^{-\kappa h_{t}}$ due to (6.8). This and (3.12) prove the lemma.

3.2.2. Local time inside the valley $\left[\tilde{L}_{j}^{-}, \tilde{L}_{j}\right]$ but far from $\tilde{m}_{j}$.

We introduce for $t>0$ and $j \geq 1$,

$$
r_{t}:=C_{0} \phi(t), \quad \mathcal{D}_{j}:=\left[\tilde{m}_{j}-r_{t}, \tilde{m}_{j}+r_{t}\right],
$$

where $C_{0}>0$ is a constant that can be chosen as large as needed. We also define

$$
\mathcal{B}_{4}(m):=\bigcap_{j=1}^{m}\left\{\sup _{x \in \overline{\mathcal{D}_{j}} \cap\left[\tilde{\tau}_{j}^{-}\left(h_{t}^{+}\right), \tilde{L}_{j}\right]}\left(\mathcal{L}\left(H\left(\tilde{L}_{j}\right), x\right)-\mathcal{L}\left(H\left(\tilde{m}_{j}\right), x\right)\right)<t e^{-2 \phi(t)}\right\}
$$

for $m \geq 1$, where $\overline{\mathcal{D}_{j}}$ is the complementary of $\mathcal{D}_{j}$. Moreover, we recall that $\tilde{L}_{j}^{-}=$ $\tilde{\tau}_{j}^{-}\left(h_{t}^{+}\right)$.

Lemma 3.4. There exists $C_{6}>0$ such that if $C_{0}$ is large enough, for large $t$,

$$
\mathbb{P}\left[\mathcal{B}_{4}\left(n_{t}\right)\right] \geq 1-C_{6} n_{t} e^{-2 \phi(t)} .
$$

Proof: Let $j \in\left[1, n_{t}\right]$. Throughout the rest of the paper, for $y \in \mathbb{R}$, we denote by $\mathbb{P}_{y}^{W_{\kappa}}$ the law of $X$ starting from $y$ instead of 0 , conditionally on $W_{\kappa}$. As we are interested in the local time at $x$ after $X$ reaches $\tilde{m}_{j}$ we work under $\mathbb{P}_{\tilde{m}_{j}}^{W_{\kappa}}$. So first, thanks to (1.12) and (1.13), under $\mathbb{P}_{\tilde{m}_{j}}^{W_{\kappa}}$, there exists a Brownian motion $(B(s), s \geq 0)$, independent of $\tilde{V}^{(j)}$, such that

$$
\mathcal{L}\left[H\left(\tilde{L}_{j}\right), x\right]=e^{-\tilde{V}^{(j)}(x)} \mathcal{L}_{B}\left[\tau^{B}\left(A^{j}\left(\tilde{L}_{j}\right)\right), A^{j}(x)\right], \quad x \in \mathbb{R},
$$

where $A^{j}(x):=\int_{\tilde{m}_{j}}^{x} e^{\tilde{V}^{(j)}(s)} \mathrm{d} s$. Let $\tilde{B}^{j}():.=B^{j}\left(\left(A^{j}\left(\tilde{L}_{j}\right)\right)^{2}\right.$.)/ $A^{j}\left(\tilde{L}_{j}\right)$. By scaling, and because $B$ is independent from $W_{\kappa}$, we notice that conditionally to $W_{\kappa}, \tilde{B}^{j}$ is a standard Brownian motion. Therefore, even if $W_{\kappa}$ appears in the expression of $\tilde{B}^{j}, \tilde{B}^{j}$ is (probabilistically) independent of $W_{\kappa}$. We still denote it by $B$ in the sequel to simplify the notation. With this notation, we have

$$
\mathcal{L}\left[H\left(\tilde{L}_{j}\right), x\right]=e^{-\tilde{V}^{(j)}(x)} A^{j}\left(\tilde{L}_{j}\right) \mathcal{L}_{B}\left[\tau^{B}(1), A^{j}(x) / A^{j}\left(\tilde{L}_{j}\right)\right], \quad x \in \mathbb{R} .
$$



duce

In order to bound the factors $\mathcal{L}_{B}\left[\tau^{B}(1),.\right]$ and $A^{j}\left(\tilde{L}_{j}\right)$ in $(3.14)$, we first intro-

$$
\mathcal{A}_{1}:=\left\{\sup _{u \in \mathbb{R}} \mathcal{L}_{B}\left[\tau^{B}(1), u\right] \leq e^{2 \phi(t)}\right\}, \quad \mathcal{A}_{2}:=\left\{A^{j}\left(\tilde{L}_{j}\right) \leq 2 e^{h_{t}+2 \phi(t) / \kappa}\right\} .
$$

We have $\mathbb{P}\left(\overline{\mathcal{A}}_{1}\right) \leq 5 e^{-2 \phi(t)}$ for large $t$ by Lemma 7.4 eq. (7.12) and (7.13). Moreover on $\mathcal{V}_{t}$, we have by Remark 2.3 and Fact 2.1 (ii) and (iii),

$$
\begin{aligned}
A^{j}\left(\tilde{L}_{j}\right) & \leq\left[\tilde{\tau}_{j}\left(h_{t}\right)-\tilde{m}_{j}\right] e^{h_{t}}+\int_{\tilde{\tau}_{j}\left(h_{t}\right)}^{\tilde{L}_{j}} e^{\tilde{V}^{(j)}(s)} \mathrm{d} s \\
& =\left[\tau_{j}\left(h_{t}\right)-m_{j}\right] e^{h_{t}}+\int_{\tau_{j}\left(h_{t}\right)}^{L_{j}} e^{V^{(j)}(s)} \mathrm{d} s \\
& \stackrel{\text { 尊 }}{=} e^{h_{t}} \tau^{W_{\kappa}^{\uparrow}}\left(h_{t}\right)+G^{+}\left(h_{t} / 2, h_{t}\right),
\end{aligned}
$$

where $W_{\kappa}^{\uparrow}$ has law $\operatorname{BES}(3, \kappa / 2)$ and is independent of $G^{+}\left(h_{t} / 2, h_{t}\right)$, which is defined in (1.7), and with $\tilde{L}_{j}=\inf \left\{s>\tilde{\tau}_{j}\left(h_{t}\right), \tilde{V}^{(j)}(s)=h_{t} / 2\right\}$ as defined in (2.4), and $L_{j}:=\inf \left\{s>\tau_{j}\left(h_{t}\right), V^{(j)}(s)=h_{t} / 2\right\}$. Consequently,

$$
\begin{aligned}
P\left(\overline{\mathcal{A}}_{2}\right) & \leq P\left(\tau^{W_{\kappa}^{\uparrow}}\left(h_{t}\right)>e^{2 \phi(t) / \kappa}\right)+P\left(G^{+}\left(h_{t} / 2, h_{t}\right)>e^{h_{t}+2 \phi(t) / \kappa}\right)+P\left(\overline{\mathcal{V}_{t}}\right) \\
& \leq C_{+} e^{-2 \phi(t)}
\end{aligned}
$$

for large $t$ by Lemma 7.2 eq. (7.5), Lemma 7.3 eq. (7.10) and Lemma 2.2, and since $\phi(t)=o(\log t)$ and $\log \log t=o(\phi(t))$.

Now, we would like to bound the factor $e^{-\tilde{V}^{(j)}(x)}$ that appears in (3.14). To this aim, let

$$
\begin{aligned}
& \mathcal{A}_{3}:=\left\{\tilde{\tau}_{j}\left[\kappa C_{0} \phi(t) / 8\right] \leq \tilde{m}_{j}+C_{0} \phi(t)\right\}, \\
& \mathcal{A}_{4}:=\left\{\inf _{\left[\tau_{j}\left[\kappa C_{0} \phi(t) / 8\right], \tau_{j}\left(h_{t}\right)\right]} V^{(j)} \geq \kappa C_{0} \phi(t) / 16\right\},
\end{aligned}
$$

with $\tilde{\tau}_{j}$ and $\tilde{\tau}_{j}^{-}$defined in (2.5) and (2.6), and $\tau_{j}$ and $\tau_{j}^{-}$in (2.1) and (2.2). First, using (6.12), $P\left(\overline{\mathcal{A}}_{3}\right) \leq C_{+} e^{-\left[\kappa^{2} C_{0} \phi(t)\right] /(16 \sqrt{2})} \leq e^{-2 \phi(t)}$ if we choose $C_{0}$ large enough. Moreover Fact 2.1 together with (7.3) (applied with $h=C_{0} \phi(t), \alpha=\kappa / 8, \gamma=\kappa / 16$ and $\omega=h_{t} /\left(C_{0} \phi(t)\right)$, see also the remark at the end of Lemma 7.2) give $P\left(\overline{\mathcal{A}}_{4}\right) \leq$ $2 e^{-\kappa^{2} C_{0} \phi(t) / 16} \leq e^{-2 \phi(t)}$ for large $t$.

We notice that $\inf _{\left[\tilde{m}_{j}+C_{0} \phi(t), \tilde{\tau}_{j}\left(h_{t}\right)\right]} \tilde{V}^{(j)} \geq \kappa C_{0} \phi(t) / 16$ on $\mathcal{A}_{3} \cap \mathcal{A}_{4} \cap \mathcal{V}_{t}$, since $\tau_{j}=\tilde{\tau}_{j}$ and $V^{(j)}=\tilde{V}^{(j)}$ on $\mathcal{V}_{t}$ thanks to Remark 2.3. We prove similarly that

$$
P\left(\overline{\mathcal{A}}_{5}\right) \leq C_{+} e^{-\kappa^{2} C_{0} \phi(t) /(16 \sqrt{2})}+P\left(\overline{\mathcal{V}_{t}}\right) \leq 2 e^{-2 \phi(t)},
$$

where

$$
\begin{aligned}
& \mathcal{A}_{5}:=\left\{\inf _{\left[\tilde{\tau}_{j}^{-}\left(h_{t}\right), \tilde{m}_{j}-C_{0} \phi(t)\right]} \tilde{V}^{(j)} \geq \kappa C_{0} \phi(t) / 16\right\}, \\
& \mathcal{A}_{6}:=\left\{\inf _{\left[\tilde{\tau}_{j}^{-}\left(h_{t}^{+}\right), \tilde{\tau}_{j}^{-}\left(h_{t}\right)\right]} \tilde{V}^{(j)} \geq h_{t} / 2\right\} .
\end{aligned}
$$

Also by (6.10), $P\left(\overline{\mathcal{A}}_{6}\right) \leq e^{-\kappa h_{t} / 8}$. We also know that $\tilde{V}^{(j)}(x) \geq h_{t} / 2 \geq \kappa C_{0} \phi(t) / 16$ for all $\tilde{\tau}_{j}\left(h_{t}\right) \leq x \leq \tilde{L}_{j}$ by definition of $\tilde{L}_{j}$, uniformly for large $t$. Consequently on $\cap_{i=3}^{6} \mathcal{A}_{i} \cap \mathcal{V}_{t}$, for all $x \in \overline{\mathcal{D}}_{j} \cap\left[\tilde{\tau}_{j}^{-}\left(h_{t}^{+}\right), \tilde{L}_{j}\right]$, we have $e^{-\tilde{V}^{(j)}(x)} \leq e^{-\kappa C_{0} \phi(t) / 16}$. 
Hence on $\cap_{i=1}^{6} \mathcal{A}_{i} \cap \mathcal{V}_{t}$, we have under $\mathbb{P}_{\tilde{m}_{j}}^{W_{\kappa}}$, by (3.14) and (3.15),

$$
\sup _{x \in \overline{\mathcal{D}_{j}} \cap\left[\tilde{\tau}_{j}^{-}\left(h_{t}^{+}\right), \tilde{L}_{j}\right]} \mathcal{L}\left[H\left(\tilde{L}_{j}\right), x\right] \leq 2 t e^{(1+2 / \kappa) \phi(t)} e^{-\kappa C_{0} \phi(t) / 16}<t e^{-2 \phi(t)},
$$

if we choose $C_{0}$ large enough. So, conditioning by $W_{\kappa}$ and applying the strong Markov property at time $H\left(\tilde{m}_{j}\right)$, we get

$$
\begin{aligned}
& \mathbb{P}\left[\sup _{x \in \overline{\mathcal{D}_{j}} \cap\left[\tilde{\tau}_{j}^{-}\left(h_{t}^{+}\right), \tilde{L}_{j}\right]}\left(\mathcal{L}\left[H\left(\tilde{L}_{j}\right), x\right]-\mathcal{L}\left[H\left(\tilde{m}_{j}\right), x\right]\right)<t e^{-2 \phi(t)}\right] \\
\geq & \mathbb{E}\left(\mathbb{P}_{\tilde{m}_{j}}^{W_{\kappa}}\left(\cap_{i=1}^{6} \mathcal{A}_{i} \cap \mathcal{V}_{t}\right)\right) \geq 1-C_{+} e^{-2 \phi(t)}
\end{aligned}
$$

uniformly for large $t$ due to the previous estimates and thanks to Lemma 2.2. This proves the lemma.

\subsection{Approximation of the main contributions.}

In this section we give an approximation of the exit time of each $h_{t}$-valley $\left[\tilde{L}_{j}^{-}, \tilde{L}_{j}\right]$ and of the local time at the bottom $\tilde{m}_{j}$ of this $h_{t}$-valley for every $1 \leq$ $j \leq n_{t}$. More precisely, we make a link between the family $\left(\left(U_{j}:=H\left(\tilde{L}_{j}\right)-\right.\right.$ $\left.\left.H\left(\tilde{m}_{j}\right), \mathcal{L}\left(H\left(\tilde{L}_{j}\right), \tilde{m}_{j}\right)\right), 1 \leq j \leq n_{t}\right)$, and the i.i.d. sequence $\left(\left(\mathcal{H}_{j}, \ell_{j}\right), 1 \leq j \leq n_{t}\right)$ described in the introduction.

In the following, $F_{1}^{+}\left(h_{t}\right), G^{+}\left(h_{t} / 2, h_{t}\right), F_{2}^{-}\left(h_{t} / 2\right)$ and $F_{3}^{-}\left(h_{t} / 2\right)$ denote independent r.v. with law respectively $F^{+}\left(h_{t}\right), G^{+}\left(h_{t} / 2, h_{t}\right), F^{-}\left(h_{t} / 2\right)$ and $F^{-}\left(h_{t} / 2\right)$, defined in (1.6) and (1.7).

Proposition 3.5. For $\delta>0$ small enough (recall that $\delta$ appears in the definitions of $n_{t}$ and $\left.h_{t}^{+}\right)$, there exist $d_{1}=d_{1}(\delta, \kappa)>0$ and $D_{1}\left(d_{1}\right)>0$ such that for large $t$, possibly on an enlarged probability space, there exists a sequence $\left(\left(S_{j}, R_{j}, \mathbf{e}_{j}\right), 1 \leq\right.$ $\left.j \leq n_{t}\right)$ of i.i.d. random variables depending on $t$, with $S_{j}, R_{j}$ and $\mathbf{e}_{j}$ independent for every $j$ and $S_{j} \stackrel{\text { 年 }}{=} F_{1}^{+}\left(h_{t}\right)+G^{+}\left(h_{t} / 2, h_{t}\right), R_{j} \stackrel{\text { 近 }}{=} F_{2}^{-}\left(h_{t} / 2\right)+F_{3}^{-}\left(h_{t} / 2\right)$ and $\mathbf{e}_{j} \stackrel{\text { II }}{=} \mathcal{E}(1 / 2)$ (exponential variable with mean 2 ) such that

$$
\mathbb{P}\left(\cap_{j=1}^{n_{t}}\left\{\left|U_{j}-\mathcal{H}_{j}\right| \leq \varepsilon_{t} \mathcal{H}_{j},\left|\mathcal{L}\left(H\left(\tilde{L}_{j}\right), \tilde{m}_{j}\right)-\ell_{j}\right| \leq \varepsilon_{t} \ell_{j}\right\}\right) \geq 1-e^{-D_{1} h_{t}}
$$

where $\ell_{j}:=S_{j} \mathbf{e}_{j}, \mathcal{H}_{j}:=R_{j} \ell_{j}$ and $\varepsilon_{t}:=e^{-d_{1} h_{t}}$.

The proof of the above proposition, which is in the spirit of the proofs of Propositions 3.4 and 4.4 in Andreoletti and Devulder (2015), makes use of the following lemma:

Lemma 3.6. For $\delta>0$ small enough, there exist constants $d_{-}>0$ and $D_{-}>0$, possibly depending on $\kappa$ and $\delta$, such that the two following statements are true for $t>0$ large enough.

(i) There exists a sequence $\left(\mathbf{e}_{j}, 1 \leq j \leq n_{t}\right)$ of i.i.d. random variables with exponential law of mean 2 and independent of $W_{\kappa}$, such that

$$
\mathbb{P}\left(\bigcap_{j=1}^{n_{t}}\left\{\left|U_{j}-\tilde{\mathbb{H}}_{j}\right| \leq e^{-\left(d_{-}\right) h_{t}} \tilde{\mathbb{H}}_{j}, \mathcal{L}\left(H\left(\tilde{L}_{j}\right), \tilde{m}_{j}\right)=\mathbb{L}_{j}\right\}\right) \geq 1-e^{-\left(D_{-}\right) h_{t}},
$$

where $\mathbb{L}_{j}:=\mathbf{e}_{j} \int_{\tilde{m}_{j}}^{\tilde{L}_{j}} e^{\tilde{V}^{(j)}(x)} \mathrm{d} x, \tilde{R}_{j}:=\int_{\tilde{\tau}_{j}^{-}\left(h_{t} / 2\right)}^{\tilde{\tau}_{j}\left(h_{t} / 2\right)} e^{-\tilde{V}^{(j)}(x)} \mathrm{d} x$ and $\tilde{\mathbb{H}}_{j}:=\mathbb{L}_{j} \tilde{R}_{j}$ for all $1 \leq j \leq n_{t}$. Moreover the random variables $\left(\mathbb{L}_{j}, \tilde{\mathbb{H}}_{j}\right), 1 \leq j \leq n_{t}$, are i.i.d. 
(ii) Possibly on an enlarged probability space, there exist random variables $R_{j}$ and $S_{j}, 1 \leq j \leq n_{t}$, such that all the random variables $R_{j}, S_{j}, \mathbf{e}_{j}, 1 \leq j \leq n_{t}$ are

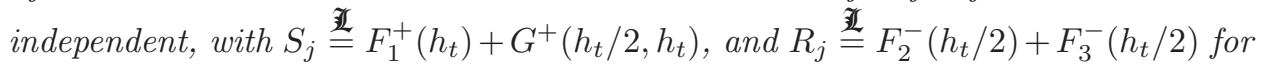
every $1 \leq j \leq n_{t}$, such that

$$
P\left(\bigcap_{j=1}^{n_{t}}\left\{\left|\int_{\tilde{m}_{j}}^{\tilde{L}_{j}} e^{\tilde{V}^{(j)}(x)} \mathrm{d} x-S_{j}\right| \leq e^{-\left(d_{-}\right) h_{t}} S_{j}, \tilde{R}_{j}=R_{j}\right\}\right) \geq 1-e^{-\left(D_{-}\right) h_{t}},
$$

Proof of Lemma 3.6: We start with (i). Recall that $\tilde{m}_{j}<\tilde{L}_{j}<\tilde{m}_{j+1}$ for every $j \geq 1$, e.g. by (2.7). By the strong Markov property applied under $\mathbb{P}^{W_{\kappa}}$ at stopping times $H\left(\tilde{m}_{j}\right)$, the random variables $\left(U_{j}, \mathcal{L}\left[H\left(\tilde{L}_{j}\right), \tilde{m}_{j}\right]\right), 1 \leq j \leq n_{t}$, are independent under $\mathbb{P}^{W_{\kappa}}$. By the same Markov property and formulas (1.12) and (1.13), the sequence $\left(U_{j}, \mathcal{L}\left[H\left(\tilde{L}_{j}\right), \tilde{m}_{j}\right], 1 \leq j \leq n_{t}\right)$ is equal to the sequence $\left(H_{j}\left(\tilde{L}_{j}\right)\right.$, $\left.\mathcal{L}_{j}\left[H_{j}\left(\tilde{L}_{j}\right), \tilde{m}_{j}\right], 1 \leq j \leq n_{t}\right)$, where

$$
\begin{aligned}
& H_{j}\left(\tilde{L}_{j}\right):=\int_{-\infty}^{\tilde{L}_{j}} e^{-\tilde{V}^{(j)}(u)} \mathcal{L}_{B^{j}}\left[\tau^{B^{j}}\left(A^{j}\left(\tilde{L}_{j}\right)\right), A^{j}(u)\right] \mathrm{d} u, \\
& \mathcal{L}_{j}\left[H_{j}\left(\tilde{L}_{j}\right), \tilde{m}_{j}\right]=\mathcal{L}_{B^{j}}\left[\tau^{B^{j}}\left(A^{j}\left(\tilde{L}_{j}\right)\right), 0\right], \quad A^{j}(u):=\int_{\tilde{m}_{j}}^{u} e^{\tilde{V}^{(j)}(x)} \mathrm{d} x, \quad u \in \mathbb{R},
\end{aligned}
$$

with $\left(B^{j}, 1 \leq j \leq n_{t}\right)$ a sequence of independent standard Brownian motions independent of $W_{\kappa}$, such that $B^{j}$ starts at $A^{j}\left(\tilde{m}_{j}\right)=0$ and is killed when it first hits $A^{j}\left(\tilde{L}_{j}\right)$. Recall that $\mathcal{L}_{B^{j}}$ denotes the local time of $B^{j}$. Define $\mathcal{A}_{j}:=$ $\left\{\max _{u<\tilde{L}_{j}^{-}} \mathcal{L}_{B^{j}}\left[\tau^{B^{j}}\left(A^{j}\left(\tilde{L}_{j}\right)\right), A^{j}(u)\right]=0\right\}, 1 \leq j \leq n_{t}$. By (6.6), there exists $c_{-}>0$ (possibly depending on $\kappa$ and $\delta$ ) such that $\mathbb{P}\left(\cap_{j=1}^{n_{t}} \mathcal{A}_{j}\right) \geq 1-e^{-\left(c_{-}\right) h_{t}}$ for large $t$. So for large $t$,

$$
\mathbb{P}\left(\bigcap_{j=1}^{n_{t}}\left\{H_{j}\left(\tilde{L}_{j}\right)=\tilde{h}_{j}\right\}\right) \geq 1-e^{-\left(c_{-}\right) h_{t}}
$$

where

$$
\tilde{h}_{j}:=\int_{\tilde{L}_{j}^{-}}^{\tilde{L}_{j}} e^{-\tilde{V}^{(j)}(u)} \mathcal{L}_{B^{j}}\left[\tau^{B^{j}}\left(A^{j}\left(\tilde{L}_{j}\right)\right), A^{j}(u)\right] \mathrm{d} u, \quad 1 \leq j \leq n_{t}
$$

We also notice that for every $1 \leq j \leq n_{t},\left(\tilde{h}_{j}, \mathcal{L}_{j}\left[H_{j}\left(\tilde{L}_{j}\right), \tilde{m}_{j}\right]\right)$ is measurable with respect to the $\sigma$-field generated by $\left(\tilde{V}^{(j)}\left(x+\tilde{L}_{j-1}^{+}\right), 0 \leq x<\tilde{L}_{j}^{+}-\tilde{L}_{j-1}^{+}\right)$and $B^{j}$, where by (2.7) and (2.8), $\tilde{L}_{j-1}^{+}<\tilde{L}_{j}^{-}<\tilde{m}_{j}<\tilde{L}_{j}<\tilde{L}_{j}^{+}$. Hence, the random variables $\left(\tilde{h}_{j}, \mathcal{L}_{j}\left[H_{j}\left(\tilde{L}_{j}\right), \tilde{m}_{j}\right]\right), 1 \leq j \leq n_{t}$ are i.i.d under $\mathbb{P}$ by the second fact of Lemma 2.2. For the same reason, $\left(\tilde{R}_{j}, A^{j}\left(\tilde{L}_{j}\right)\right), 1 \leq j \leq n_{t}$ are also i.i.d.

For $1 \leq j \leq n_{t}$, let $\tilde{B}^{j}():.=B^{j}\left(\left(A^{j}\left(\tilde{L}_{j}\right)\right)^{2}.\right) / A^{j}\left(\tilde{L}_{j}\right)$. Notice that

$$
\mathcal{L}_{B^{j}}\left[\tau^{B^{j}}\left(A^{j}\left(\tilde{L}_{j}\right)\right), A^{j}(u)\right]=A^{j}\left(\tilde{L}_{j}\right) \mathcal{L}_{\tilde{B}^{j}}\left[\tau^{\tilde{B}^{j}}(1), A^{j}(u) / A^{j}\left(\tilde{L}_{j}\right)\right], \quad \tilde{L}_{j}^{-} \leq u \leq \tilde{L}_{j}
$$

Moreover by scaling and because $B^{j}$ is independent from $W_{\kappa}, \tilde{B}^{j}$ is, conditionally to $W_{\kappa}$, a standard Brownian motion starting from 0 and killed when it first hits 1 . 
Furthermore, even if $W_{\kappa}$ appears in the expression of $\tilde{B}^{j}, \tilde{B}^{j}$ is independent of $W_{\kappa}$. Then, let

$$
\mathbf{e}_{j}:=\mathcal{L}_{\tilde{B}^{j}}\left[\tau^{\tilde{B}^{j}}(1), 0\right]=\mathcal{L}_{B^{j}}\left[\tau^{B^{j}}\left(A^{j}\left(\tilde{L}_{j}\right)\right), 0\right] / A^{j}\left(\tilde{L}_{j}\right) .
$$

Notice that by the first Ray-Knight theorem, $\mathbf{e}_{j}$ is exponentially distributed with mean 2. Since $\tilde{B}^{j}$ is independent of $W_{\kappa}, \mathbf{e}_{j}$ is also independent of $W_{\kappa}$. Also, the sequence $\mathbf{e}_{j}, 1 \leq j \leq n_{t}$ is i.i.d. because the $B^{j}$ are independent and the $\left(\tilde{R}_{j}, A^{j}\left(\tilde{L}_{j}\right)\right)$ are i.i.d., so $\left(\mathbb{L}_{j}, \tilde{\mathbb{H}}_{j}\right), 1 \leq j \leq n_{t}$, are also i.i.d. Moreover, (3.21) leads to

$$
\mathcal{L}_{j}\left[H_{j}\left(\tilde{L}_{j}\right), \tilde{m}_{j}\right]=A^{j}\left(\tilde{L}_{j}\right) \mathcal{L}_{\tilde{B}^{j}}\left[\tau^{\tilde{B}^{j}}(1), 0\right]=A^{j}\left(\tilde{L}_{j}\right) \mathbf{e}_{j}=\mathbb{L}_{j} .
$$

Now, for small $\varepsilon>0$, thanks to Lemma 6.3 , we have for large $t$,

$$
\begin{aligned}
\mathbb{P}\left(\bigcap_{j=1}^{n_{t}}\left\{\left|\tilde{h}_{j}-A^{j}\left(\tilde{L}_{j}\right) \mathbf{e}_{j} \tilde{R}_{j}\right| \leq 2 e^{-(1-3 \varepsilon) h_{t} / 6} A^{j}\left(\tilde{L}_{j}\right) \mathbf{e}_{j} \tilde{R}_{j}\right\}\right) & \geq 1-\frac{C_{+} n_{t}}{e^{(c-) \varepsilon h_{t}}} \\
& \geq 1-\frac{C_{+}}{e^{(c-/ 2) \varepsilon h_{t}}},
\end{aligned}
$$

since $n_{t}=e^{o(1) h_{t}}$. Finally, this, together with (3.20), (3.23) and the equality of sequences at the start of this proof show (3.17) for some $D_{-}>0$ and $d_{-}>0$. So (i) is proved.

We now prove (ii). The r.v. $\tilde{A}_{j}\left(\tilde{L}_{j}\right)=\int_{\tilde{m}_{j}}^{\tilde{L}_{j}} e^{\tilde{V}^{(j)}(x)} \mathrm{d} x$ and $\tilde{R}_{j}$ are not independent, so we want to replace them by r.v. having better independence properties. Applying Lemma 6.4 with subscript 2 replaced by $j$ for $1 \leq j \leq n_{t}$ gives the existence of $R_{j}$ and $S_{j}$, independent and independent of $\mathbf{e}_{\mathbf{j}}$, having the law claimed in (ii) and satisfying (6.5) with 2 replaced by $j$. This gives (3.18) since $n_{t}=e^{o(1) h_{t}}$. The fact that we can build these $R_{j}$ and $S_{j}$ with the claimed independence properties follows from the fact that $\left(\mathbf{e}_{\mathbf{j}}, \tilde{R}_{j}, \tilde{A}^{j}\left(\tilde{L}_{j}\right)\right), 1 \leq j \leq n_{t}$ are i.i.d.

Proof of Proposition 3.5: The existence and the law of the $\mathbf{e}_{\mathbf{j}}$ come from Lemma 3.6 (i). The existence and the law of the $R_{j}$ and $S_{j}$, and the independence of $R_{j}$, $S_{j}, \mathbf{e}_{\mathbf{j}}, 1 \leq j \leq n_{t}$ come from Lemma 3.6 (ii). Moreover, by Lemma 3.6 (i) and (ii), there exist $d_{1}>0$ and $D_{1}>0$ such that for large $t$,

$$
\begin{aligned}
& \mathbb{P}\left(\cap_{j=1}^{n_{t}}\left\{\left|U_{j}-\mathbf{e}_{j} S_{j} R_{j}\right| \leq \varepsilon_{t} \mathbf{e}_{j} S_{j} R_{j},\left|\mathcal{L}\left(H\left(\tilde{L}_{j}\right), \tilde{m}_{j}\right)-\mathbf{e}_{j} S_{j}\right| \leq \varepsilon_{t} \mathbf{e}_{j} S_{j}\right\}\right) \\
\geq & 1-e^{-D_{1} h_{t}}
\end{aligned}
$$

which proves (3.16). So Proposition 3.5 is proved.

\section{Convergence toward the Lévy process $\left(\mathcal{Y}_{1}, \mathcal{Y}_{2}\right)$ and continuity}

4.1. Preliminaries. We begin this section by the convergence of certain repartition functions. These key results are in the same spirit as the second part of Lemma 5.1 in Andreoletti and Devulder (2015). 
Lemma 4.1. Recall from Proposition 3.5 that $\ell_{1}:=\mathbf{e}_{1} S_{1}$ and $\mathcal{H}_{1}:=\mathbf{e}_{1} S_{1} R_{1}$. Then for any $\varepsilon \in(0,1 / 3)$,

$$
\begin{aligned}
& \lim _{t \rightarrow+\infty} \sup _{x \in\left[e^{-(1-2 \varepsilon) \phi(t)},+\infty[\right.}\left|x^{\kappa} e^{\kappa \phi(t)} \mathbb{P}\left(\ell_{1} / t>x\right)-\mathcal{C}_{2}\right|=0, \\
& \lim _{t \rightarrow+\infty} \sup _{y \in\left[e^{-(1-3 \varepsilon) \phi(t)},+\infty[\right.}\left|y^{\kappa} e^{\kappa \phi(t)} \mathbb{P}\left(\mathcal{H}_{1} / t>y\right)-\mathcal{C}_{2} \mathbb{E}\left[\left(\mathcal{R}_{\kappa}\right)^{\kappa}\right]\right|=0,
\end{aligned}
$$

with $\mathcal{C}_{2}$ a positive constant (see below (4.10)).

Moreover, for any $\alpha>0, e^{\kappa \phi(t)} \mathbb{P}\left(\ell_{1} / t \geq x, \mathcal{H}_{1} / t \geq y\right)$ converges uniformly when $t$ goes to infinity on $[\alpha,+\infty[\times[\alpha,+\infty[$ to $\nu([x,+\infty[\times[y,+\infty[)$, where $\nu$ is defined in (1.4).

Proof: Let $\varepsilon \in(0,1 / 3)$.

Proof of (4.1): We first prove that, as $t \rightarrow+\infty, x^{\kappa} e^{\kappa \phi(t)} \mathbb{P}\left(S_{1} / t>x\right)$ converges uniformly in $x \in\left[e^{-(1-\varepsilon) \phi(t)},+\infty[\right.$ to a constant $c$, that is, we prove that

$$
\lim _{t \rightarrow+\infty} \sup _{x \in\left[e^{-(1-\varepsilon) \phi(t)},+\infty[\right.}\left|x^{\kappa} e^{\kappa \phi(t)} \mathbb{P}\left(S_{1} / t>x\right)-c\right|=0 .
$$

For that, with the change of variables $y=e^{(1-\varepsilon) \phi(t)} x$, we just have to prove that

$$
\lim _{t \rightarrow+\infty} \sup _{y \in[1,+\infty[}\left|y^{\kappa} e^{\kappa \varepsilon \phi(t)} \mathbb{P}\left(S_{1} / e^{h_{t}+\varepsilon \phi(t)}>y\right)-c\right|=0,
$$

but this is equivalent to prove that for any function $f:] 0,+\infty[\rightarrow[1,+\infty[$,

$$
\lim _{t \rightarrow+\infty}(f(t))^{\kappa} e^{\kappa \varepsilon \phi(t)} \mathbb{P}\left(S_{1} / e^{h_{t}+\varepsilon \phi(t)}>f(t)\right)=c .
$$

First by definition (see Proposition 3.5), $S_{1}$ can be written as the sum of two independent random variables, that we denote by $F_{1}^{+}\left(h_{t}\right)$ and $G^{+}\left(h_{t} / 2, h_{t}\right)$ for simplicity. That is,

$$
S_{1} / t=\left(F_{1}^{+}\left(h_{t}\right)+G^{+}\left(h_{t} / 2, h_{t}\right)\right) / t=e^{-\phi(t)}\left(e^{-h_{t}} F_{1}^{+}\left(h_{t}\right)+e^{-h_{t}} G^{+}\left(h_{t} / 2, h_{t}\right)\right) .
$$

Since we know the asymptotic behavior of the Laplace transforms of $F^{+}\left(h_{t}\right) / e^{h_{t}}$ and $G^{+}\left(h_{t} / 2, h_{t}\right) / e^{h_{t}}$, the proof of (4.5) is similar to the proof of a Tauberian theorem. First by (7.1) and (7.2) we have, using the independence of $F_{1}^{+}\left(h_{t}\right)$ and $G^{+}\left(h_{t} / 2, h_{t}\right)$,

$$
\begin{aligned}
\forall \gamma>0, \quad \omega_{f, t}(\gamma) \quad:= & \frac{1}{\gamma}\left(1-\mathbb{E}\left[e^{-\gamma S_{1} /\left(f(t) e^{h_{t}+\varepsilon \phi(t)}\right)}\right]\right) \\
& \sim \underset{t \rightarrow+\infty}{\sim} c^{\prime} \gamma^{\kappa-1}(f(t))^{-\kappa} e^{-\kappa \varepsilon \phi(t)},
\end{aligned}
$$

where $c^{\prime}=\Gamma(1-\kappa) 2^{\kappa} / \Gamma(1+\kappa)$. Note that by Fubini, $\omega_{f, t}$ is the Laplace transform of the measure $\mathrm{d} U_{f, t}(z):=\mathbb{1}_{\mathbb{R}_{+}}(z) \mathbb{P}\left(S_{1} /\left(f(t) e^{h_{t}+\varepsilon \phi(t)}\right)>z\right) \mathrm{d} z$, that is, $\omega_{f, t}(\gamma)=$ $\int_{0}^{\infty} e^{-\gamma z} \mathrm{~d} U_{f, t}(z)$. From (4.7), we have

$$
\forall \gamma>0, \quad \frac{\omega_{f, t}(\gamma)}{\omega_{f, t}(1)} \underset{t \rightarrow+\infty}{\longrightarrow} \gamma^{\kappa-1} .
$$

We can now follow the same line as in the proof of a classical Tauberian theorem, making the link between a Laplace transform and the repartition function, (see for 
example Feller, 1971 volume 2, section XIII.5, Theorem 1, page 442), we can deduce that

$$
\forall z>0, \quad \frac{U_{f, t}([0, z])}{\omega_{f, t}(1)} \underset{t \rightarrow+\infty}{\longrightarrow} \frac{z^{1-\kappa}}{\Gamma(2-\kappa)} .
$$

Then, e.g. as in the proof of Theorem 4 of the same reference page 446 , or using inequalities similar to those at the end of the proof of Lemma 5.1 in Andreoletti and Devulder (2015), we deduce from the monotony of the densities of measures $U_{f, t}$ that

$$
\forall z>0, \quad \frac{\mathbb{P}\left(S_{1} /\left(f(t) e^{h_{t}+\varepsilon \phi(t)}\right)>z\right)}{\omega_{f, t}(1)} \underset{t \rightarrow+\infty}{\longrightarrow} z^{-\kappa} \frac{1-\kappa}{\Gamma(2-\kappa)} .
$$

Considering this convergence with $z=1$ we get exactly (4.5) for $c=c^{\prime}(1-\kappa) / \Gamma(2-$ $\kappa)=2^{\kappa} / \Gamma(1+\kappa)$, so $(4.3)$ follows.

Now, let $a_{t}:=e^{\varepsilon \phi(t)}$. For any $x>0$,

$$
x^{\kappa} e^{\kappa \phi(t)} \mathbb{P}\left(\mathbf{e}_{1} S_{1} / t>x, \mathbf{e}_{1}<a_{t}\right)=2^{-1} \int_{0}^{a_{t}}(x / u)^{\kappa} e^{\kappa \phi(t)} \mathbb{P}\left(S_{1} / t>x / u\right) u^{\kappa} e^{-u / 2} \mathrm{~d} u,
$$

because $\mathbf{e}_{1}$ has law $\mathcal{E}(1 / 2)$ and is independent of $S_{1}$.

Taking $x$ arbitrary in $\left[e^{-(1-2 \varepsilon) \phi(t)},+\infty\right.$, we have $x / u \in\left[e^{-(1-\varepsilon) \phi(t)},+\infty\right.$ [ for every $\left.u \in] 0, a_{t}\right]$, so thanks to $(4.3)$ we get

$$
\lim _{t \rightarrow+\infty} \sup _{x \in\left[e^{-(1-2 \varepsilon) \phi(t)},+\infty\right.}\left|x^{\kappa} e^{\kappa \phi(t)} \mathbb{P}\left(\mathbf{e}_{1} S_{1} / t>x, \mathbf{e}_{1}<a_{t}\right)-\frac{c}{2} \int_{0}^{+\infty} \frac{u^{\kappa}}{e^{u / 2}} \mathrm{~d} u\right|=0 .
$$

Now for $t$ large enough such that $\forall y \geq 1, y^{\kappa} e^{\kappa \phi(t)} \mathbb{P}\left(S_{1} / t>y\right)<2 c$ (see (4.3)), we have for any $x>0$,

$$
\begin{aligned}
& \left|x^{\kappa} e^{\kappa \phi(t)} \mathbb{P}\left(\mathbf{e}_{1} S_{1} / t>x, \mathbf{e}_{1}<a_{t}\right)-x^{\kappa} e^{\kappa \phi(t)} \mathbb{P}\left(\mathbf{e}_{1} S_{1} / t>x\right)\right| \\
& =x^{\kappa} e^{\kappa \phi(t)} \mathbb{P}\left(\mathbf{e}_{1} S_{1} / t>x, \mathbf{e}_{1} \geq a_{t}\right) \\
& =2^{-1} \int_{a_{t}}^{+\infty} x^{\kappa} e^{\kappa \phi(t)} \mathbb{P}\left(S_{1} / t>x / u\right) e^{-u / 2} \mathrm{~d} u \\
& =2^{-1} \int_{a_{t}}^{+\infty} u^{\kappa}(x / u)^{\kappa} e^{\kappa \phi(t)} \mathbb{P}\left(S_{1} / t>x / u\right) \mathbb{1}_{x \leq u} e^{-u / 2} \mathrm{~d} u \\
& +2^{-1} \int_{a_{t}}^{+\infty} u^{\kappa}(x / u)^{\kappa} e^{\kappa \phi(t)} \mathbb{P}\left(S_{1} / t>x / u\right) \mathbb{1}_{x>u} e^{-u / 2} \mathrm{~d} u \\
& \leq 2^{-1} e^{\kappa \phi(t)} \int_{a_{t}}^{+\infty} u^{\kappa} e^{-u / 2} \mathrm{~d} u+c \int_{a_{t}}^{+\infty} u^{\kappa} e^{-u / 2} \mathrm{~d} u
\end{aligned}
$$

For the second term in the inequality we used the fact that

$$
(x / u)^{\kappa} e^{\kappa \phi(t)} \mathbb{P}\left(S_{1} / t>x / u\right)<2 c
$$

when $x \geq u$. Since $a_{t}=e^{\varepsilon \phi(t)}$, the right hand side of (4.9) converges to 0 when $t$ goes to infinity. Combining this with (4.8), we get

$$
\lim _{t \rightarrow+\infty} \sup _{x \in\left[e^{-(1-2 \varepsilon) \phi(t)},+\infty[\right.}\left|x^{\kappa} e^{\kappa \phi(t)} \mathbb{P}\left(\mathbf{e}_{1} S_{1} / t>x\right)-2^{-1} c \int_{0}^{+\infty} u^{\kappa} e^{-u / 2} \mathrm{~d} u\right|=0,
$$


and this is exactly (4.1) with $\mathcal{C}_{2}:=2^{-1} c \int_{0}^{+\infty} u^{\kappa} e^{-u / 2} \mathrm{~d} u=2^{\kappa} \Gamma(\kappa+1) c=4^{\kappa}$.

Proof of (4.2): Let $\mu_{R_{1}}$ be the distribution of $R_{1}$. For any $y>0, a>0$ and $t>0$, we have by independence of $\mathbf{e}_{1} S_{1}$ and $R_{1}$,

$y^{\kappa} e^{\kappa \phi(t)} \mathbb{P}\left(\mathbf{e}_{1} S_{1} R_{1} / t>y, R_{1}<a\right)=\int_{0}^{a}(y / u)^{\kappa} e^{\kappa \phi(t)} \mathbb{P}\left(\mathbf{e}_{1} S_{1} / t>y / u\right) u^{\kappa} \mu_{R_{1}}(\mathrm{~d} u)$.

Taking $a=a_{t}=e^{\varepsilon \phi(t)}$ and $y$ arbitrary in $\left[e^{-(1-3 \varepsilon) \phi(t)},+\infty[\right.$, we have $y / u \in$ $\left[e^{-(1-2 \varepsilon) \phi(t)},+\infty[\right.$ for all $\left.u \in] 0, a_{t}\right]$, so thanks to $(4.10)$, we get

$\lim _{t \rightarrow+\infty} \sup _{y \in\left[e^{-(1-3 \varepsilon) \phi(t)},+\infty[\right.}\left|y^{\kappa} e^{\kappa \phi(t)} \mathbb{P}\left(\mathbf{e}_{1} S_{1} R_{1} / t>y, R_{1}<a_{t}\right)-\mathcal{C}_{2} \int_{0}^{a_{t}} u^{\kappa} \mu_{R_{1}}(\mathrm{~d} u)\right|$

$=0$

where we used $\int_{0}^{\infty} u^{\kappa} \mu_{R_{1}}(\mathrm{~d} u)=\mathbb{E}\left[\left(R_{1}\right)^{\kappa}\right] \leq \mathbb{E}\left[\left(\mathcal{R}_{\kappa}\right)^{\kappa}\right]<\infty$, as explained in the following lines. By definition (see before Proposition 3.5 and (1.6)) and with $\widetilde{W_{\kappa}^{\uparrow}}$ an independent copy of $W_{\kappa}^{\uparrow}, R_{1}$ is equal in law to $\int_{0}^{\tau^{W_{\kappa}^{\uparrow}}\left(h_{t} / 2\right)} e^{-W_{\kappa}^{\uparrow}(x)} \mathrm{d} x+$ $\int_{0}^{\tau^{\widetilde{W}_{\kappa}^{\uparrow}}\left(h_{t} / 2\right)} e^{-\widetilde{W}_{\kappa}^{\uparrow}(x)} \mathrm{d} x$, which itself converges almost surely to $\mathcal{R}_{\kappa}$ (defined in (1.2)) when $t$ goes to infinity. This also shows that for each $t, R_{1}$ is stochastically inferior to $\mathcal{R}_{\kappa}$, which admits finite moments of any positive order by Lemma 6.6. In particular the family $\left(R_{1}\right)_{t>0}$ is bounded in all $L^{p}$ spaces, and more precisely, $\mathbb{E}\left[\left(R_{1}\right)^{p}\right] \leq \mathbb{E}\left[\left(\mathcal{R}_{\kappa}\right)^{p}\right]<\infty$ for every $p \in \mathbb{R}_{+}$. So by the dominated convergence theorem, $\int_{0}^{+\infty} u^{\kappa} \mu_{R_{1}}(\mathrm{~d} u)$ converges to $\mathbb{E}\left[\left(\mathcal{R}_{\kappa}\right)^{\kappa}\right]$ when $t$ goes to infinity. Hence,

$$
\lim _{t \rightarrow+\infty} \sup _{y \in\left[e^{-(1-3 \varepsilon) \phi(t)},+\infty[\right.}\left|y^{\kappa} e^{\kappa \phi(t)} \mathbb{P}\left(\mathbf{e}_{1} S_{1} R_{1} / t>y, R_{1}<a_{t}\right)-\mathcal{C}_{2} \mathbb{E}\left[\left(\mathcal{R}_{\kappa}\right)^{\kappa}\right]\right|=0 .
$$

Finally, as the family $\left(R_{1}\right)_{t>0}$ is bounded in all $L^{p}$ spaces, $e^{\kappa \phi(t)} \int_{a_{t}}^{\infty} u^{\kappa} \mu_{R_{1}}(\mathrm{~d} u)$ converges to 0 as $t \rightarrow+\infty$. So we can proceed as before (as in (4.9), integrating with respect to $R_{1}$ instead of $\mathbf{e}_{1}$ and using (4.1) instead of (4.3)) to remove the event $R_{1}<a_{t}$ and we thus get

$$
\lim _{t \rightarrow+\infty} \sup _{y \in\left[e^{-(1-3 \varepsilon) \phi(t)},+\infty[\right.}\left|y^{\kappa} e^{\kappa \phi(t)} \mathbb{P}\left(\mathbf{e}_{1} S_{1} R_{1} / t>y\right)-\mathcal{C}_{2} \mathbb{E}\left[\left(\mathcal{R}_{\kappa}\right)^{\kappa}\right]\right|=0,
$$

which is (4.2). We now prove the last assertion. For any $x>0, y>0, a>0$ and $t>0$, we have

$$
\begin{aligned}
& e^{\kappa \phi(t)} \mathbb{P}\left(\mathbf{e}_{1} S_{1} / t>x, \mathbf{e}_{1} S_{1} R_{1} / t>y, R_{1}<a\right) \\
&= \int_{0}^{a} e^{\kappa \phi(t)} \mathbb{P}\left(\mathbf{e}_{1} S_{1} / t>x, \mathbf{e}_{1} S_{1} / t>y / u\right) \mu_{R_{1}}(\mathrm{~d} u) \\
&= \int_{0}^{a \wedge(y / x)} e^{\kappa \phi(t)} \mathbb{P}\left(\mathbf{e}_{1} S_{1} / t>y / u\right) \mu_{R_{1}}(\mathrm{~d} u) \\
&+\int_{a \wedge(y / x)}^{a} e^{\kappa \phi(t)} \mathbb{P}\left(\mathbf{e}_{1} S_{1} / t>x\right) \mu_{R_{1}}(\mathrm{~d} u), \\
&= \frac{1}{y^{\kappa}} \int_{0}^{a \wedge(y / x)} e^{\kappa \phi(t)}(y / u)^{\kappa} \mathbb{P}\left(\mathbf{e}_{1} S_{1} / t>y / u\right) u^{\kappa} \mu_{R_{1}}(\mathrm{~d} u) \\
& \quad+\frac{1}{x^{\kappa}} \int_{a \wedge(y / x)}^{a} e^{\kappa \phi(t)} x^{\kappa} \mathbb{P}\left(\mathbf{e}_{1} S_{1} / t>x\right) \mu_{R_{1}}(\mathrm{~d} u) .
\end{aligned}
$$


Taking $a=a_{t}=e^{\varepsilon \phi(t)}$ and $x, y$ arbitrary in $[\alpha,+\infty[$ (for some $\alpha>0$ ), we have $(y / u, x) \in\left[e^{-(1-2 \varepsilon) \phi(t)},+\infty\left[{ }^{2}, \forall u \in\right] 0, a_{t}\right]$ whenever $t$ is large enough, so, thanks to (4.10) we get that $e^{\kappa \phi(t)} \mathbb{P}\left(\mathbf{e}_{1} S_{1} / t>x, \mathbf{e}_{1} S_{1} R_{1} / t>y, R_{1}<a_{t}\right)$ converges uniformly in $(x, y) \in[\alpha,+\infty[\times[\alpha,+\infty[$ toward

$$
\mathcal{C}_{2} x^{-\kappa} \mathbb{P}\left(\mathcal{R}_{\kappa}>y / x\right)+\mathcal{C}_{2} y^{-\kappa} \mathbb{E}\left(\left(\mathcal{R}_{\kappa}\right)^{\kappa} \mathbb{1}_{\mathcal{R}_{\kappa} \leq y / x}\right)=\nu([x,+\infty[\times[y,+\infty[) .
$$

Then as before we can remove the event $\left\{R_{1}<a_{t}\right\}$ since $e^{\kappa \phi(t)} \mathbb{P}\left(R_{1} \geq a_{t}\right) \rightarrow 0$ as $t \rightarrow+\infty$ because the family $\left(R_{1}\right)_{t>0}$ is bounded in all $L^{p}$ spaces, which gives the last assertion of Lemma 4.1.

\subsection{Proof of Proposition 1.4.}

We start with the finite dimensional convergence. We recall that $\left(Y_{1}, Y_{2}\right)_{s}^{t}$ is defined just before Proposition 1.4, and $\left(\mathcal{Y}_{1}, \mathcal{Y}_{2}\right)$ before (1.4). We sometimes use the notation $\left(\mathcal{Y}_{1}, \mathcal{Y}_{2}\right)_{s}=\left(\mathcal{Y}_{1}(s), \mathcal{Y}_{2}(s)\right)$ and $\left(Y_{1}, Y_{2}\right)_{s}^{t}=\left(Y_{1}^{t}(s), Y_{2}^{t}(s)\right)$.

Lemma 4.2. For any $k \in \mathbb{N}$ and $s_{i}>0, i \leq k,\left(\left(Y_{1}, Y_{2}\right)_{s_{i}}^{t}, i \leq k\right)$ converges in law as $t$ goes to infinity to $\left(\left(\mathcal{Y}_{1}, \mathcal{Y}_{2}\right)_{s_{i}}, i \leq k\right)$.

Proof: The proof is basic here, however we give some details as we deal with a two dimensional walk which increments depend on $t$ itself. As $Y_{1}^{t}(s)$ and $Y_{2}^{t}(s)$ are sums of i.i.d sequences we only have to prove the convergence in law for the couple $\left(Y_{1}, Y_{2}\right)_{s}^{t}$ for any $s>0$. For $b \geq 0$, we define $\left(Y_{1}^{>b}, Y_{2}^{>b}\right)$, obtained from $\left(Y_{1}, Y_{2}\right)^{t}$ by keeping only the increments larger than $b$, that is, $Y_{1}^{>b}(s):=\frac{1}{t} \sum_{j=1}^{\left\lfloor s e^{\kappa \phi(t)}\right\rfloor} \ell_{j} \mathbb{1}_{\ell_{j} / t>b}$ and $Y_{2}^{>b}(s):=\frac{1}{t} \sum_{j=1}^{\left\lfloor s e^{\kappa \phi(t)}\right\rfloor} \mathcal{H}_{j} \mathbb{1}_{\mathcal{H}_{j} / t>b}$ for every $s \geq 0$ and $t>0$. Also let $Y_{i}^{\leq b}(s):=$ $Y_{i}^{t}(s)-Y_{i}^{>b}(s)$ for $i \in\{1,2\}$. We first prove that for any $s>0$,

$$
\lim _{\varepsilon \rightarrow 0} \limsup _{t \rightarrow+\infty} \mathbb{P}\left(\left\|\left(Y_{1}^{\leq \varepsilon}, Y_{2}^{\leq \varepsilon}\right)_{s}^{t}\right\|>\varepsilon^{1-\kappa(2-\kappa)}\right)=0,
$$

where for any $a=\left(a_{1}, a_{2}\right) \in \mathbb{R}^{2},\|a\|:=\max \left(\left|a_{1}\right|,\left|a_{2}\right|\right)$, with $\left(Y_{1}^{\leq \varepsilon}, Y_{2}^{\leq \varepsilon}\right)_{s}^{t}=$ $\left(Y_{1}^{\leq \varepsilon}(s), Y_{2}^{\leq \varepsilon}(s)\right)$ and $1-\kappa(2-\kappa)>0$ since $\kappa<1$.

Let $\varepsilon>0$ and $s>0$. We now give an upper bound for the first moments of $Y_{1}^{\leq \varepsilon}(s)$ and $Y_{2}^{\leq \varepsilon}(s)$. Let $\eta>0$ be such that $\kappa-(1-3 \eta)<0$. Applying Fubini, we have for large $t$,

$$
\begin{aligned}
& e^{\kappa \phi(t)} \mathbb{E}\left(\frac{\ell_{1}}{t} \mathbb{1}_{\ell_{1} / t \leq \varepsilon}\right) \\
= & e^{\kappa \phi(t)} \mathbb{E}\left[\frac{\mathbf{e}_{1} S_{1}}{t} \mathbb{1}_{\mathbf{e}_{1} S_{1} / t \leq \varepsilon}\right] \\
\leq & \int_{0}^{\varepsilon} e^{\kappa \phi(t)} \mathbb{P}\left(\mathbf{e}_{1} S_{1} / t>x\right) \mathrm{d} x \\
= & \int_{0}^{e^{-(1-2 \eta) \phi(t)}} e^{\kappa \phi(t)} \mathbb{P}\left(\mathbf{e}_{1} S_{1} / t>x\right) \mathrm{d} x+\int_{e^{-(1-2 \eta) \phi(t)}}^{\varepsilon} e^{\kappa \phi(t)} \mathbb{P}\left(\mathbf{e}_{1} S_{1} / t>x\right) \mathrm{d} x \\
\leq & e^{(\kappa-(1-2 \eta)) \phi(t)}+\int_{e^{-(1-2 \eta) \phi(t)}}^{\varepsilon} x^{-\kappa} x^{\kappa} e^{\kappa \phi(t)} \mathbb{P}\left(\mathbf{e}_{1} S_{1} / t>x\right) \mathrm{d} x .
\end{aligned}
$$

The first term in (4.13) converges to 0 when $t$ goes to infinity because $\kappa-(1-2 \eta)<$ $-\eta<0$. Moreover, according to (4.1), for $t$ large enough, we have

$$
\forall x \geq e^{-(1-2 \eta) \phi(t)}, \quad x^{\kappa} e^{\kappa \phi(t)} \mathbb{P}\left(\mathbf{e}_{1} S_{1} / t>x\right) \leq 2 \mathcal{C}_{2} .
$$


For such $t$, the second term in (4.13) is less than

$$
2 \mathcal{C}_{2} \int_{0}^{\varepsilon} x^{-\kappa} \mathrm{d} x=2 \mathcal{C}_{2} \frac{\varepsilon^{1-\kappa}}{1-\kappa}
$$

So, we get for large $t$,

$$
e^{\kappa \phi(t)} \mathbb{E}\left(\frac{\ell_{1}}{t} \mathbb{1}_{\ell_{1} / t \leq \varepsilon}\right) \leq e^{(\kappa-(1-2 \eta)) \phi(t)}+C_{+} \varepsilon^{1-\kappa} .
$$

Using the same method and applying this time (4.2), we get for large $t$,

$$
e^{\kappa \phi(t)} \mathbb{E}\left(\frac{\mathcal{H}_{1}}{t} \mathbb{1}_{\mathcal{H}_{1} / t \leq \varepsilon}\right) \leq e^{(\kappa-(1-3 \eta)) \phi(t)}+C_{+} \varepsilon^{1-\kappa}
$$

We thus obtain

$$
\begin{aligned}
& \mathbb{E}\left(Y_{1}^{\leq \varepsilon}(s)\right) \leq s e^{(\kappa-(1-2 \eta)) \phi(t)}+C_{+} s \varepsilon^{1-\kappa}, \\
& \mathbb{E}\left(Y_{2}^{\leq \varepsilon}(s)\right) \leq s e^{(\kappa-(1-3 \eta)) \phi(t)}+C_{+} s \varepsilon^{1-\kappa},
\end{aligned}
$$

then a Markov inequality leads to $(4.12)$ since $\kappa-(1-3 \eta)<0$.

The next step is to prove that $\left(Y_{1}^{>\varepsilon}, Y_{2}^{>\varepsilon}\right)_{s}^{t}$ can be written as the integral of a point process which converges to the desired limit. We have

$$
\left(Y_{1}^{>\varepsilon}, Y_{2}^{>\varepsilon}\right)_{s}^{t}=\left(Y_{1}^{>\varepsilon}(s), Y_{2}^{>\varepsilon}(s)\right)=\left(\int_{x>\varepsilon} \int_{0}^{s} x \mathcal{P}_{t}^{1}(\mathrm{~d} x, \mathrm{~d} v), \int_{x>\varepsilon} \int_{0}^{s} x \mathcal{P}_{t}^{2}(\mathrm{~d} x, \mathrm{~d} v)\right)
$$

where the measures $\mathcal{P}_{t}^{1}$ and $\mathcal{P}_{t}^{2}$ are defined by $\mathcal{P}_{t}^{1}:=\sum_{i=1}^{+\infty} \delta_{\left(t^{-1} \ell_{i}, e^{-\kappa \phi(t)} i\right)}$ and similarly $\mathcal{P}_{t}^{2}:=\sum_{i=1}^{+\infty} \delta_{\left(t^{-1} \mathcal{H}_{i}, e^{-\kappa \phi(t)} i\right)}$. Recall that $\mathcal{P}_{t}^{1}$ and $\mathcal{P}_{t}^{2}$ are not independent. We now prove that $\left(\mathcal{P}_{t}^{1}, \mathcal{P}_{t}^{2}\right)$ converges to a Poisson point measure. For that just use Lemma 4.1 together with Proposition 3.1 in Resnick (1986) after discretization, it implies that $\left(\mathcal{P}_{t}^{1}, \mathcal{P}_{t}^{2}\right)$ converges weakly to the Poisson random measure denoted by $\left(\mathcal{P}^{1}, \mathcal{P}^{2}\right)$ with intensity measure given by $\mathrm{d} s \times \nu$.

Then using that for any $\varepsilon>0$, and $T<+\infty$, on $[0, T) \times(\varepsilon,+\infty) \times(\varepsilon,+\infty)$ $\mathrm{d} s \times \nu$ is finite, we have that $\left(Y_{1}^{>\varepsilon}, Y_{2}^{>\varepsilon}\right)_{s}^{t}$ converges weakly to

$$
\left(\mathcal{Y}_{1}^{>\varepsilon}, \mathcal{Y}_{2}^{>\varepsilon}\right)_{s}:=\left(\int_{x>\varepsilon} \int_{0}^{s} x \mathcal{P}^{1}(\mathrm{~d} x, \mathrm{~d} v), \int_{x>\varepsilon} \int_{0}^{s} x \mathcal{P}^{2}(\mathrm{~d} x, \mathrm{~d} v)\right) \text {. }
$$

We are left to prove that $\left(\mathcal{Y}_{1}^{>\varepsilon}, \mathcal{Y}_{2}^{>\varepsilon}\right)$ converges to $\left(\mathcal{Y}_{1}, \mathcal{Y}_{2}\right)$ when $\varepsilon \downarrow 0$. This is a straightforward computation, that we detail for completeness. Let $\nu_{1}([x,+\infty[):=$ $\nu\left(\left[x,+\infty\left[\times \mathbb{R}_{+}\right)=\mathcal{C}_{2} / x^{\kappa}\right.\right.$, we have

$$
\mathbb{E}\left(\int_{x \leq \varepsilon} \int_{0}^{s} x \mathcal{P}^{1}(\mathrm{~d} x, \mathrm{~d} v)\right)=s \int_{x \leq \varepsilon} x \nu_{1}(\mathrm{~d} x)=C \varepsilon^{1-\kappa},
$$

Then a Markov inequality proves that for any $s>0$, the process $\int_{x \leq \varepsilon} \int_{0}^{s} x \mathcal{P}^{1}(\mathrm{~d} x, \mathrm{~d} v)$ converges to zero (when $\varepsilon$ goes to zero) in probability. The same is true for $\int_{x \leq \varepsilon} \int_{0}^{s} x \mathcal{P}^{2}(\mathrm{~d} x, \mathrm{~d} v)$, so we obtain that $\left(\mathcal{Y}_{1}^{>\varepsilon}, \mathcal{Y}_{2}^{>\varepsilon}\right)_{s}$ converges in probability to $\left(\mathcal{Y}_{1}, \mathcal{Y}_{2}\right)_{s}$ when $\varepsilon \rightarrow 0$.

We now prove the tightness of $\left(\mathcal{D}\left(Y_{1}, Y_{2}\right)^{t}\right)_{t}$, the family of measures induced by processes $\left(Y_{1}, Y_{2}\right)^{t}$.

Lemma 4.3. The family of laws $\left(\mathcal{D}\left(Y_{1}, Y_{2}\right)^{t}\right)_{t}$ is tight on $\left(D\left([0,+\infty), \mathbb{R}^{2}\right), J_{1}\right)$. 
Proof: We only have to prove that the family law of the restriction of the process to the interval $[0, T],\left(\left.\left(Y_{1}, Y_{2}\right)^{t}\right|_{[0, T]}\right)_{t}$ is tight. To prove this we use the following restatement of Theorem 1.8 in Billingsley (1999) using Aldous's tightness criterion (see Condition 1, and equation (16.22) page 176 in Billingsley, 1999) also used in Bovier (2010) page 100. We have to check the two following statements:

1 ) for any $\varepsilon>0$, there exists $a$ such that for any $t$ large enough,

$$
\mathbb{P}\left(\sup _{s \in[0, T]}\left\|\left(Y_{1}, Y_{2}\right)_{s}^{t}\right\| \geq a\right) \leq \varepsilon .
$$

2) for any $\varepsilon>0$, and $\eta>0$ there exists $\delta, 0<\delta<T$ and $t_{0}>0$ such that for $t>t_{0}$

$$
\mathbb{P}\left[\omega\left(\left(Y_{1}, Y_{2}\right)^{t}, \delta, T\right) \geq \eta\right] \leq \varepsilon,
$$

with $\omega\left(\left(Y_{1}, Y_{2}\right)^{t}, \delta, T\right):=\sup _{0 \leq r \leq T} \omega\left(\left(Y_{1}, Y_{2}\right)^{t}, \delta, T, r\right)$, and

$$
\begin{aligned}
& \omega\left(\left(Y_{1}, Y_{2}\right)^{t}, \delta, T, r\right) \\
& :=\sup _{0 \vee(r-\delta) \leq u_{1}<u<u_{2} \leq(r+\delta) \wedge T}\left(\operatorname { m i n } \left\{\left\|\left(Y_{1}, Y_{2}\right)_{u_{2}}^{t}-\left(Y_{1}, Y_{2}\right)_{u}^{t}\right\|,\right.\right. \\
& \left.\left.\left\|\left(Y_{1}, Y_{2}\right)_{u}^{t}-\left(Y_{1}, Y_{2}\right)_{u_{1}}^{t}\right\|\right\}\right) .
\end{aligned}
$$

Also

$$
\mathbb{P}\left(v\left(\left(Y_{1}, Y_{2}\right)^{t}, 0, \delta, T\right) \geq \eta\right) \leq \varepsilon, \text { and } \mathbb{P}\left(v\left(\left(Y_{1}, Y_{2}\right)^{t}, T, \delta, T\right) \geq \eta\right) \leq \varepsilon,
$$

where $v\left(\left(Y_{1}, Y_{2}\right)^{t}, u, \delta, T\right):=\sup _{(u-\delta) \vee 0 \leq u_{1} \leq u_{2} \leq(u+\delta) \wedge T}\left\{\left\|\left(Y_{1}, Y_{2}\right)_{u_{1}}^{t}-\left(Y_{1}, Y_{2}\right)_{u_{2}}^{t}\right\|\right\}$.

We first check 1) since the process is monotone increasing,

$$
\mathbb{P}\left(\sup _{s \in[0, T]}\left\|\left(Y_{1}, Y_{2}\right)_{s}^{t}\right\| \geq a\right)=\mathbb{P}\left(\left\|\left(Y_{1}, Y_{2}\right)_{T}^{t}\right\| \geq a\right) \leq \mathbb{P}\left(Y_{1}(T) \geq a\right)+\mathbb{P}\left(Y_{2}(T) \geq a\right) .
$$

Recall that $Y_{1}^{>b}$ is obtained from $Y_{1}$ where we remove the increments $\ell_{j} / t$ smaller than $b$ and $Y_{1}^{\leq b}=Y_{1}-Y_{1}^{>b}$. Define $N_{u}^{>b}:=\sum_{i=1}^{\left\lfloor u e^{\kappa \phi(t)}\right\rfloor} \mathbb{1}_{\ell_{j} / t>b}$. Let $0<\delta_{1}<1$. A Markov inequality yields

$$
\begin{aligned}
\mathbb{P}\left(Y_{1}^{t}(T) \geq a\right) & \leq \mathbb{P}\left(Y_{1}^{\leq 1}(T) \geq \frac{a}{2}\right)+\mathbb{P}\left(Y_{1}^{>1}(T) \geq \frac{a}{2}\right) \\
& \leq \frac{2}{a} \mathbb{E}\left[Y_{1}^{\leq 1}(T)\right]+\frac{1}{a^{\delta_{1}}} \mathbb{E}\left(N_{T}^{>1}\right)+\mathbb{P}\left(Y_{1}^{>1}(T) \geq \frac{a}{2}, N_{T}^{>1} \leq a^{\delta_{1}}\right)
\end{aligned}
$$

On $\left\{N_{T}^{>1} \leq a^{\delta_{1}}\right\}$ there is at most $a^{\delta_{1}}$ terms in the sum $Y_{1}^{>1}(T)$ so

$$
\begin{aligned}
\mathbb{P}\left(Y_{1}^{>1}(T)>a / 2, N_{T}^{>1} \leq a^{\delta_{1}}\right) & \leq \sum_{1 \leq i \leq a^{\delta_{1}}} \mathbb{P}\left(\ell_{i} / t \geq\left(a^{1-\delta_{1}} / 2\right) \mid \ell_{i} / t \geq 1\right) \\
& \leq a^{\delta_{1}} \mathbb{P}\left(\ell_{1} / t \geq\left(a^{1-\delta_{1}} / 2\right) \mid \ell_{1} / t \geq 1\right) \\
& \leq a^{\delta_{1}} 2 \frac{\mathcal{C}_{2} e^{-\kappa \phi(t)} a^{-\kappa\left(1-\delta_{1}\right)} 2^{\kappa}}{\mathcal{C}_{2} e^{-\kappa \phi(t)}} \\
& =2^{1+\kappa} a^{\delta_{1}-\kappa\left(1-\delta_{1}\right)},
\end{aligned}
$$

for all $t$ large enough thanks to $(4.1)$ and $\delta_{1}$ such that $\delta_{1}-\kappa\left(1-\delta_{1}\right)<0$. 
Also, notice that for any $b>0, N_{T}^{>b}$ follows a binomial law with parameters $\left(\left\lfloor T e^{\kappa \Phi(t)}\right\rfloor, \mathbb{P}\left(\ell_{1} / t>b\right)\right)$. So, using (4.1) again and (4.16), we obtain for $t$ large enough,

$$
\mathbb{E}\left(N_{T}^{>b}\right) \leq 2 \mathcal{C}_{2} T b^{-\kappa}, \quad \mathbb{E}\left[Y_{1}^{\leq b}(T)\right] \leq 2 \mathcal{C}_{2} T b^{1-\kappa} .
$$

Collecting (4.20), (4.21) and (4.19), we get the existence of $t_{1}>0$ such that

$$
\lim _{a \rightarrow+\infty} \sup _{t \geq t_{1}} \mathbb{P}\left(Y_{1}(T) \geq a\right)=0 .
$$

The same arguments holds for $Y_{2}$ (using (4.2) instead of (4.1) and (4.17) instead of (4.16)) so (4.22) also holds for $Y_{2}$ instead of $Y_{1}$. We conclude the proof of 1 ) by putting (4.22) and its analogous for $Y_{2}$ in (4.18).

We now check 2) We first write, as usual,

$$
\begin{aligned}
& \left\{\omega\left(\left(Y_{1}, Y_{2}\right)^{t}, \delta, T\right) \geq \eta\right\} \\
\subset \quad & \left\{\omega\left(\left(Y_{1}^{\leq b}, Y_{2}^{\leq b}\right)^{t}, \delta, T\right) \geq \eta / 2\right\} \cup\left\{\omega\left(\left(Y_{1}^{>b}, Y_{2}^{>b}\right)^{t}, \delta, T\right) \geq \eta / 2\right\} .
\end{aligned}
$$

For $Y \leq b$, we have

$$
\mathbb{P}\left[\omega\left(\left(Y_{1}^{\leq b}, Y_{2}^{\leq b}\right)^{t}, \delta, T\right) \geq \eta / 2\right] \leq \mathbb{P}\left[\omega\left(Y_{1}^{\leq b}, \delta, T\right) \geq \eta / 2\right]+\mathbb{P}\left[\omega\left(Y_{2}^{\leq b}, \delta, T\right) \geq \eta / 2\right] .
$$

Moreover, by positivity of the increments,

$$
\begin{aligned}
& \mathbb{P}\left(\omega\left(Y_{1}^{\leq b}, \delta, T\right) \geq \eta / 2\right) \\
\leq & \mathbb{P}\left(\cup_{k \leq\lfloor T / 2 \delta\rfloor}\left\{Y_{1}^{\leq b}((k+1) 2 \delta)-Y_{1}^{\leq b}(k 2 \delta) \geq \eta / 4\right\}\right) \\
\leq & \sum_{k \leq\lfloor T / \delta\rfloor} \mathbb{P}\left(Y_{1}^{\leq b}((k+1) 2 \delta)-Y_{1}^{\leq b}(k 2 \delta) \geq \eta / 4\right) .
\end{aligned}
$$

For any $k, Y_{1}^{\leq b}((k+1) 2 \delta)-Y_{1}^{\leq b}(k 2 \delta)$ is the sum of at most $\left\lfloor 2 \delta e^{\kappa \Phi(t)}\right\rfloor+1$ i.i.d. random variables having the same law as $\ell_{1} / t$. We get that for any integer $k$

$$
\mathbb{P}\left(Y_{1}^{\leq b}((k+1) 2 \delta)-Y_{1}^{\leq b}(k 2 \delta) \geq \eta / 4\right) \leq \mathbb{P}\left(Y_{1}^{\leq b}(3 \delta) \geq \eta / 4\right) \leq 8 \mathcal{C}_{2} \delta b^{1-\kappa} / \eta,
$$

where the first inequality holds for $t$ large enough so that $2 \delta e^{\kappa \Phi(t)} \geq 1$ and the second from the second expression in (4.21) (replacing $T$ by $2 \delta$ ). Combining with (4.23) we get for large $t$

$$
\mathbb{P}\left(\omega\left(Y_{1}^{\leq b}, \delta, T\right) \geq \eta / 2\right) \leq 24 \mathcal{C}_{2} T(1+2 \delta) b^{1-\kappa} / \eta,
$$

[note that $\delta$ will be chosen later (and will be less than 1)]. $T$ and $\eta$ are fixed so we choose $b$ small enough so that the right hand side of (4.23) is less than $\varepsilon / 4$. A similar estimate can be proved for $\mathbb{P}\left(\omega\left(Y_{2}^{\leq b}, \delta, T\right) \geq \eta / 2\right)$.

For $Y^{>b}$, we have again

$$
\mathbb{P}\left(\omega\left(\left(Y_{1}^{>b}, Y_{2}^{>b}\right)^{t}, \delta, T\right) \geq \eta / 2\right) \leq \mathbb{P}\left(\omega\left(Y_{1}^{>b}, \delta, T\right) \geq \eta / 2\right)+\mathbb{P}\left(\omega\left(Y_{2}^{>b}, \delta, T\right) \geq \eta / 2\right) .
$$

Since $Y_{1}^{>b}$ is piecewise constant with jumps larger than $b,\left\{\omega\left(Y_{1}^{>b}, \delta, T\right)>\eta / 2\right\}$ implies that two jumps larger than $b$ for $Y_{1}^{t}$ occur in an interval smaller than 
$2 \delta$. That is $\left\{\omega\left(Y_{1}^{>b}, \delta, T\right)>\eta / 2\right\} \subset \cup_{j=1}^{\left\lfloor T e^{\kappa \phi(t)}\right\rfloor} \cup_{i>j,(i-j) / e^{\kappa \phi(t)} \leq 2 \delta}^{\left\lfloor T e^{\kappa \phi(t)}\right.}\left\{\ell_{j} \wedge \ell_{i} / t>b\right\}$. Applying (4.1) for $t$ large enough,

$$
\mathbb{P}\left(\cup_{j=1}^{\left\lfloor T e^{\kappa \phi(t)}\right\rfloor} \cup_{i>j,(i-j) / e^{\kappa \phi(t)} \leq 2 \delta}^{\left\lfloor T e^{\kappa(t)}\right.}\left\{\ell_{j} \wedge \ell_{i} / t>b\right\}\right) \leq 8 \mathcal{C}_{2}^{2} \delta T b^{-2 \kappa},
$$

which can be small choosing this time $\delta=\delta(b)$ properly. Again the same argument can be used for $\omega\left(Y_{2}^{>b}, \delta, T\right)$. To finish the proof, we have to deal with $v()$, as again our processes are increasing,

$$
\mathbb{P}\left(v\left(\left(Y_{1}, Y_{2}\right)^{t}, 0, \delta, T\right) \geq \eta\right) \leq \mathbb{P}\left(\left\|\left(Y_{1}, Y_{2}\right)_{\delta}^{t}\right\| \geq \eta\right)
$$

we can then proceed as for 1) decreasing the value of $\delta$ if needed, this also applies to $\mathbb{P}\left(v\left(\left(Y_{1}, Y_{2}\right)^{t}, T, \delta, T\right) \geq \eta\right)$.

Putting together the two preceding lemmata we obtain Proposition 1.4.

4.3. Continuity of some functionals of $\left(\mathcal{Y}_{1}, \mathcal{Y}_{2}\right)$ in $J_{1}$ topology. In this section, we study the continuity of some functionals which will be applied later to $\left(Y_{1}, Y_{2}\right)^{t}$ and to the Lévy processes $\left(\mathcal{Y}_{1}, \mathcal{Y}_{2}\right)$.

For our purpose, we are interested in the following mappings. We have already mentioned the first two in the introduction:

$$
\begin{aligned}
J: D\left(\mathbb{R}_{+}, \mathbb{R}\right) & \longrightarrow D\left(\mathbb{R}_{+}, \mathbb{R}\right) & I:\left(D\left(\mathbb{R}_{+}, \mathbb{R}\right), J_{1}\right) & \longrightarrow\left(D\left(\mathbb{R}_{+}, \mathbb{R}\right), U\right) \\
f & \longmapsto f^{\natural} & f & \longmapsto f^{-1}
\end{aligned}
$$

where $U$ denotes uniform convergence on every compact subset of $\mathbb{R}_{+}$. Then we also need the compositions of these two: for any positive $a$, let

$$
\begin{aligned}
& J_{I, a}: \quad D\left(\mathbb{R}_{+}, \mathbb{R}^{2}\right) \quad \longrightarrow \mathbb{R} \quad J_{I, a}^{-}: D\left(\mathbb{R}_{+}, \mathbb{R}^{2}\right) \longrightarrow \mathbb{R} \\
& f=\left(f_{1}, f_{2}\right) \longmapsto f_{1}^{\natural}\left(f_{2}^{-1}(a)\right), \quad f=\left(f_{1}, f_{2}\right) \longmapsto f_{1}^{\natural}\left(f_{2}^{-1}(a)^{-}\right) \text {, }
\end{aligned}
$$

$J_{I, a}$ (respectively $J_{I, a}^{-}$) produces the largest jump of $f_{1}$, between 0 and the time just after (respectively before) $f_{2}$ first reaches $(a,+\infty)$. We also define $K_{I, a}, K_{I, a}^{-}$, $\tilde{K}_{I, a}$ and $\tilde{K}_{I, a}^{-}$as follows.

$$
\begin{aligned}
& K_{I, a}: \quad D\left(\mathbb{R}_{+}, \mathbb{R}^{2}\right) \longrightarrow \mathbb{R} \\
& f=\left(f_{1}, f_{2}\right) \longmapsto f_{1}\left(f_{2}^{-1}(a)\right), \\
& K_{I, a}^{-}: \quad D\left(\mathbb{R}_{+}, \mathbb{R}^{2}\right) \quad \longrightarrow \mathbb{R} \\
& f=\left(f_{1}, f_{2}\right) \longmapsto f_{1}\left(f_{2}^{-1}(a)^{-}\right), \\
& \tilde{K}_{I, a}: \quad D\left(\mathbb{R}_{+}, \mathbb{R}^{2}\right) \longrightarrow \mathbb{R} \\
& f=\left(f_{1}, f_{2}\right) \longmapsto f_{2}\left(f_{2}^{-1}(a)\right), \\
& \tilde{K}_{I, a}^{-}: \quad D\left(\mathbb{R}_{+}, \mathbb{R}^{2}\right) \longrightarrow \mathbb{R} \\
& f=\left(f_{1}, f_{2}\right) \longmapsto f_{2}\left(f_{2}^{-1}(a)^{-}\right) .
\end{aligned}
$$

Finally, with $\Delta f_{1}(s):=f_{1}(s)-f_{1}\left(s^{-}\right)$, define $F^{*}$ by

$$
\begin{aligned}
F^{*}: & D\left(\mathbb{R}_{+}, \mathbb{R}^{2}\right) \\
f=\left(f_{1}, f_{2}\right) & \longmapsto \operatorname{Rinf}\left\{s \in\left(0, f_{2}^{-1}(1)\right), \Delta f_{1}(s)=f_{1}^{\natural}\left(f_{2}^{-1}(1)^{-}\right)\right\} .
\end{aligned}
$$

We need this functional $F^{*}$ for the characterization of the favorite site.

Lemma 4.4. $J$ is continuous in the $J_{1}$ topology. 
Proof: This fact is basic. However, we have not found a proof in the literature, so we give some details. To prove the continuity on $D\left(\mathbb{R}_{+}, \mathbb{R}\right)$, we only have to prove it for every compact subset of $\mathbb{R}_{+}$, (see Whitt, 2002 Theorem 12.9.1). So let $f \in D\left(\mathbb{R}_{+}, \mathbb{R}\right)$ and $T>0$ at which $f$ is continuous, let us prove that $J_{T}$ defined by

$$
\begin{aligned}
& J_{T}: \quad D([0, T], \mathbb{R}) \longrightarrow D([0, T], \mathbb{R}) \\
& g \quad \longmapsto g^{\natural}
\end{aligned}
$$

is continuous at the restriction $f_{\mid[0, T]}$. Let $\varepsilon>0$ and $g \in D([0, T], \mathbb{R})$ such that $d_{T}\left(f_{\mid[0, T]}, g\right) \leq \frac{\varepsilon}{2}$. $d_{T}$ is the usual metric $d$ of the $J_{1}$-topology restricted to the interval $[0, T]$. By definition of $d_{T}$ there exists a strictly increasing continuous mapping of $[0, T]$ onto itself, $e:[0, T] \longrightarrow[0, T]$ such that

$$
\sup _{s \in[0, T]}|e(s)-s| \leq \frac{\varepsilon}{2} \text { and } \sup _{s \in[0, T]}\left|g(e(s))-f_{\mid[0, T]}(s)\right| \leq \frac{\varepsilon}{2} .
$$

So for every $s \in[0, T]$ we have

$$
\begin{aligned}
\left|\Delta g(e(s))-\Delta f_{\mid[0, T]}(s)\right| & =\left|(g(e(s))-g(e(s)-))-\left(f_{\mid[0, T]}(s)-f_{\mid[0, T]}(s-)\right)\right| \\
& \leq\left|g(e(s))-f_{\mid[0, T]}(s)\right|+\left|g(e(s)-)-f_{\mid[0, T]}(s-)\right| \\
& \leq 2 \frac{\varepsilon}{2}=\varepsilon,
\end{aligned}
$$

where $\Delta h(s)=h(s)-h\left(s^{-}\right)$. This implies $d_{T}\left(J_{T}\left(f_{\mid[0, T]}\right), J_{T}(g)\right) \leq \varepsilon$.

Lemma 4.5. Fix $a>0$. The mappings $J_{I, a}^{-}, J_{I, a}, K_{I, a}^{-}, K_{I, a}, \tilde{K}_{I, a}^{-}$and $\tilde{K}_{I, a}$ are continuous for $J_{1}$-topology at every couple $\left(f^{1}, f^{2}\right) \in D\left(\mathbb{R}_{+}, \mathbb{R}^{2}\right)$ such that

(1) For any $\varepsilon>0, f^{1}$ and $f^{2}$ have a finite number of jumps greater than $\varepsilon$ on every compact subset of $\mathbb{R}_{+}^{*}$,

(2) $f^{2}$ is strictly increasing, with a limit equal to $+\infty$,

(3) $f^{2}(0)=0$,

(4) $f^{2}$ has a jump at $I\left(f^{2}\right)(a)$ and $f^{2}\left(I\left(f^{2}\right)(a)-\right)<a<f^{2}\left(I\left(f^{2}\right)(a)\right)$.

Proof: This fact may also be known as we are looking at randomly stopped process, but once again we did not find what we need in the literature (Silvestrov, 2008, Whitt, 2002).

Let $\left(f_{n}^{1}, f_{n}^{2}\right)_{n}$ be a sequence of elements of $D\left(\mathbb{R}_{+}, \mathbb{R}\right)$ which converges to $\left(f^{1}, f^{2}\right)$ for the $J_{1}$ topology. To prove continuity, we prove that the sequence $\left(J_{I, a}^{-}\left(f_{n}^{1}, f_{n}^{2}\right)\right)_{n}$ converges to $J_{I, a}^{-}\left(f^{1}, f^{2}\right)$, and the equivalent for $J_{I, a}$.

The first hypothesis guaranties that there exist neighborhoods of $I\left(f^{2}\right)(a)$ for which $f^{1}$ makes no jump greater than $1 / 4$ times its higher previous jump, that is to say there exists $\delta \in] 0, I\left(f^{2}\right)(a)\left[\right.$ (notice that $I\left(f^{2}\right)(a)$ exists tanks to $(2)$ and is positive thanks to (3)) such that $f^{1}$ makes no jump greater than $J\left(f^{1}\right)\left(I\left(f^{2}\right)(a)-\delta\right) / 4$ on $\left[I\left(f^{2}\right)(a)-\delta, I\left(f_{2}\right)(a)[\right.$ and on $\left.] I\left(f^{2}\right)(a), I\left(f^{2}\right)(a)+\delta\right]$. Note also that $J\left(f^{1}\right)$ is constant on $\left[I\left(f^{2}\right)(a)-\delta, I\left(f_{2}\right)(a)[\right.$ and on $\left.] I\left(f^{2}\right)(a), I\left(f^{2}\right)(a)+\delta\right]$.

Also $\delta$ can be made smaller (if needed) in such a way that $I\left(f^{2}\right)(a)+\delta$ is a point of continuity of $\left(f^{1}, f^{2}\right)$ and $\left(f_{n}^{1}, f_{n}^{2}\right)_{n}$ for every $n \in \mathbb{N}$. By hypothesis 


$$
\begin{aligned}
& d\left(\left(f_{n}^{1}, f_{n}^{2}\right),\left(f^{1}, f^{2}\right)\right) \longrightarrow_{n \rightarrow+\infty} 0 \text { so } \\
& \quad d_{n}:=d_{\left[0, I\left(f^{2}\right)(a)+\delta\right]}\left(\left(f_{n}^{1}, f_{n}^{2}\right)_{\mid\left[0, I\left(f^{2}\right)(a)+\delta\right]},\left(f^{1}, f^{2}\right)_{\mid\left[0, I\left(f^{2}\right)(a)+\delta\right]}\right) \longrightarrow_{n \rightarrow+\infty} 0,
\end{aligned}
$$

where $\mid\left[0, I\left(f^{2}\right)(a)+\delta\right]$ in index means restriction to $\left[0, I\left(f^{2}\right)(a)+\delta\right]$. Also by continuity of $J$ (see Lemma 4.4) we also have $d\left(J\left(f_{n}^{1}\right), J\left(f^{1}\right)\right) \longrightarrow_{n \rightarrow+\infty} 0$ and therefore

$$
d_{n}^{\prime}:=d_{\left[0, I\left(f^{2}\right)(a)+\delta\right]}\left(\left(J\left(f_{n}^{1}\right)\right)_{\mid\left[0, I\left(f^{2}\right)(a)+\delta\right]},\left(J\left(f^{1}\right)\right)_{\mid\left[0, I\left(f^{2}\right)(a)+\delta\right]}\right) \longrightarrow_{n \rightarrow+\infty} 0 .
$$

Let $h^{-}$(respectively $h^{+}$) be the largest jump of $f^{1}$ just before (resp. just after) $I\left(f^{2}\right)(a)$. By definition of $\delta$ we have

$$
h^{-}=J\left(f^{1}\right)\left(I\left(f^{2}\right)(a)-\delta\right), h^{+}=J\left(f^{1}\right)\left(I\left(f^{2}\right)(a)+\delta\right) .
$$

We have two cases, either $J\left(f^{1}\right)$ is continuous at $I\left(f^{2}\right)(a)$ or it makes a jump. Case $J\left(f^{1}\right)$ makes a jump, in this case the size of the jump is $h^{+}-h^{-}>0$.

Let $\alpha=8^{-1} \min \left(h^{-}, \delta, 1-f^{2}\left(I\left(f^{2}\right)(a)^{-}\right), f^{2}\left(I\left(f^{2}\right)(a)\right)-1\right)$, and $n_{0} \in \mathbb{N}$ be such that for any $n \geq n_{0}, d_{n}<\alpha$ and $d_{n}^{\prime}<\alpha$. T $=I\left(f^{2}\right)(a)+\delta$, there exist two homeomorphisms $e_{n}, e_{n}^{\prime}:[0, T] \longrightarrow[0, T]$ such that:

$$
\begin{aligned}
& \text { - } \sup _{s \in[0, T]}\left|e_{n}(s)-s\right| \leq d_{n}, \\
& \text { - }\left.\sup _{s \in[0, T]}||\left(f_{n}^{1}\left(e_{n}(s)\right), f_{n}^{2}\left(e_{n}(s)\right)\right)_{\mid\left[0, I\left(f^{2}\right)(a)+\delta\right]}\right|_{\mid} \leq d_{n} . \\
& \qquad-\left.\left(f^{1}(s), f^{2}(s)\right)_{\mid\left[0, I\left(f^{2}\right)(a)+\delta\right]}\right|_{\infty} \sup _{s \in[0, T]}\left|e_{n}^{\prime}(s)-s\right| \leq d_{n}^{\prime}, \\
& \text { - } \sup _{s \in[0, T]}\left|\left(J\left(f_{n}^{1}\right)\right)_{\mid\left[0, I\left(f^{2}\right)(a)+\delta\right]}\left(e_{n}^{\prime}(s)\right)-\left(J\left(f^{1}\right)\right)_{\mid\left[0, I\left(f^{2}\right)(a)+\delta\right]}(s)\right| \leq d_{n}^{\prime} .
\end{aligned}
$$

The second inequality implies that for any $n \geq n_{0}$,

$$
f_{n}^{2}\left(e_{n}\left(I\left(f^{2}\right)(a)^{-}\right)\right)<a<f_{n}^{2}\left(e_{n}\left(I\left(f^{2}\right)(a)\right)\right),
$$

so as we also have $f_{n}^{2}\left(I\left(f_{n}^{2}\right)(a)^{-}\right) \leq a \leq f_{n}^{2}\left(I\left(f_{n}^{2}\right)(a)\right)$ we get

$$
I\left(f_{n}^{2}\right)(a)=e_{n}\left(I\left(f^{2}\right)(a)\right) .
$$

The fourth point implies that for any $n \geq n_{0}$,

$$
J\left(f_{n}^{1}\right)\left(e_{n}^{\prime}\left(I\left(f^{2}\right)(a)-\frac{1}{2} \delta\right)\right) \geq J\left(f^{1}\right)\left(I\left(f^{2}\right)(a)-\frac{1}{2} \delta\right)-\alpha=h^{-}-\alpha>\frac{1}{2} h^{-} .
$$

The second point and the argument of the previous proof imply that for any $n \geq$ $n_{0}$, each jump of $f_{n}^{1}$ on $\left[e_{n}\left(I\left(f^{2}\right)(a)-\delta\right), e_{n}\left(I\left(f^{2}\right)(a)\right)\right.$ [ is $2 \alpha$-close to a jump of $f^{1}$ on $\left[I\left(f^{2}\right)(a)-\delta, I\left(f^{2}\right)(a)\left[\right.\right.$, but such jumps are less than $h^{-} / 4$ because of the definition of $\delta$. Thus, $f_{n}^{1}$ makes no jump larger than $h^{-} / 2$ on the interval $\left[e_{n}\left(I\left(f^{2}\right)(a)-\delta\right), e_{n}\left(I\left(f^{2}\right)(a)\right)\right)$. Moreover, the increases of $e_{n}^{\prime}$ and the first and third points imply that

$$
e_{n}\left(I\left(f^{2}\right)(a)-\delta\right) \leq e_{n}^{\prime}\left(I\left(f^{2}\right)(a)-\delta / 2\right) \leq e_{n}\left(I\left(f^{2}\right)(a)\right) .
$$

So, combining this with (4.27), we get that $J\left(f_{n}^{1}\right)$ is constant on the interval $\left[e_{n}^{\prime}\left(I\left(f^{2}\right)(a)-\delta / 2\right), e_{n}\left(I\left(f^{2}\right)(a)\right)\right)$. 
Now by definition of $J_{I, a}^{-}$, with (4.26) and then collecting what have just done above yields

$$
\begin{aligned}
\forall n \geq n_{0}, \quad J_{I, a}^{-}\left(\left(f_{n}^{1}, f_{n}^{2}\right)\right) & =J\left(f_{n}^{1}\right)\left(I\left(f_{n}^{2}\right)(a)^{-}\right)=J\left(f_{n}^{1}\right)\left(e_{n}\left(I\left(f^{2}\right)(a)\right)^{-}\right) \\
& =J\left(f_{n}^{1}\right)\left(e_{n}^{\prime}\left(I\left(f^{2}\right)(a)-\delta / 2\right)\right) .
\end{aligned}
$$

From definition of $J_{I, a}^{-}$and the constantness of $J\left(f^{1}\right)$ on $\left[I\left(f^{2}\right)(a)-\delta, I\left(f_{2}\right)(a)[\right.$ we also have

$$
J_{I, a}^{-}\left(f^{1}, f^{2}\right):=J\left(f^{1}\right)\left(I\left(f^{2}\right)(a)-\right)=J\left(f^{1}\right)\left(I\left(f^{2}\right)(a)-\delta / 2\right) .
$$

Combining (4.28), (4.29) and the fourth point gives that, as $n$ goes to infinity, $J_{I, a}^{-}\left(\left(f_{n}^{1}, f_{n}^{2}\right)\right)$ converges to $J_{I, a}^{-}\left(\left(f^{1}, f^{2}\right)\right)$.

For $J_{I, a}$, we prove in a similar way as above that $J\left(f_{n}^{1}\right)$ is constant on $\left[e_{n}\left(I\left(f^{2}\right)(a)\right), e_{n}^{\prime}\left(I\left(f^{2}\right)(a)+\delta / 2\right)\right]$ so, as in (4.28) we have for $n$ large enough

$$
J_{I, a}\left(\left(f_{n}^{1}, f_{n}^{2}\right)\right)=J\left(f_{n}^{1}\right)\left(e_{n}^{\prime}\left(I\left(f^{2}\right)(a)+\delta / 2\right)\right),
$$

which, combined with the analogous of (4.29)

$$
J_{I, a}\left(f^{1}, f^{2}\right)=J\left(f^{1}\right)\left(I\left(f^{2}\right)(a)+\delta / 2\right)
$$

allows us to conclude, using the fourth point, that $J_{I, a}\left(\left(f_{n}^{1}, f_{n}^{2}\right)\right)$ converges to $J_{I, a}\left(\left(f^{1}, f^{2}\right)\right)$ as $n$ goes to infinity. Therefore, both $J_{I, a}^{-}$and $J_{I, a}$ are continue at $\left(f^{1}, f^{2}\right)$. The continuity of the other functionals are proved similarly.

Lemma 4.6. For any $\left(f^{1}, f^{2}\right)$ in $D\left(\mathbb{R}_{+}, \mathbb{R}^{2}\right)$ that satisfy the hypothesis of lemma 4.5 and such that the sizes of the jumps of $f^{1}$ are all distinct, $F^{*}$ is continuous at $\left(f^{1}, f^{2}\right)$ in the $J_{1}$ topology.

Proof: The proof follows mainly the steps of Lemma 4.5, we keep the same notation. The jump which takes place at the instant $F^{*}\left(f^{1}, f^{2}\right)$ has value $h^{-}$. With the additional hypothesis that the values of the jumps for $f^{1}$ are all different we have unicity for the value $h^{-}$. Let us define $h^{\prime}$, the second highest jump $f^{1}$ before instant $I\left(f^{2}\right)(1)$. With the additional condition that $\alpha<\frac{1}{8}\left(h^{-}-h^{\prime}\right)$ we have with the same arguments as in the proof of the continuity of $J$ that for any $n \geq n_{0}, f_{n}^{1}$ effectuates at $e_{n}\left(F^{*}\left(f^{1}, f^{2}\right)\right)$ a jump larger than $h^{-}-2 \alpha$, and larger than all the other jumps of $f_{n}^{1}$ before $e_{n}\left(I\left(f^{2}\right)(1)-\right)=I\left(f_{n}^{2}\right)(1)$ which are smaller than $h^{\prime}+2 \alpha$. So for $n \geq n_{0}$, the largest jump of $f^{1}$ before $I\left(f_{n}^{2}\right)(1)$ is obtained for $e_{n}\left(F^{*}\left(f^{1}, f^{2}\right)\right)$, that is to say for any $n \geq n_{0}$,

$$
F^{*}\left(\left(f_{n}^{1}, f_{n}^{2}\right)\right)=e_{n}\left(F^{*}\left(f^{1}, f^{2}\right)\right)
$$

this implies $F^{*}\left(\left(f_{n}^{1}, f_{n}^{2}\right)\right) \longrightarrow_{n \rightarrow \infty} F^{*}\left(f^{1}, f^{2}\right)$.

\section{Supremum of the Local time - and other functionals}

5.1. Supremum of the local time (proof of Theorem 1.3).

First, notice that since the diffusion $X$ is almost surely transient to the right, the random variable $\sup _{x<0} \mathcal{L}(+\infty, x)$ is $\mathbb{P}$-almost surely finite. So almost surely,

$$
\lim _{t \rightarrow+\infty} \sup _{x<0} \mathcal{L}(t, x) / t=0 .
$$

As a consequence, we only have to study the asymptotic behavior of $\sup _{x \geq 0} \mathcal{L}(t, x) / t$ as $t \rightarrow+\infty$. 
We start with the proof of the following proposition, which makes a link between the supremum of the local time and the process $\left(Y_{1}, Y_{2}\right)^{t}$.

Proposition 5.1. Let $\alpha>0$. For any $\varepsilon>0$ and large $t$,

$$
\mathcal{P}_{1}^{-}-v(\varepsilon, t) \leq \mathbb{P}\left(\sup _{x \geq 0} \mathcal{L}(t, x) / t \leq \alpha\right) \leq \mathcal{P}_{1}^{+}+v(\varepsilon, t),
$$

where

$$
\mathcal{P}_{1}^{ \pm}:=\mathbb{P}\left[\left(1-\overline{\mathcal{H}}_{\mathcal{N}_{t}^{2 \varepsilon}-1}\right) \frac{\bar{\ell}_{\mathcal{N}_{t}^{2 \varepsilon}}-\bar{\ell}_{\mathcal{N}_{t}^{2 \varepsilon}-1}}{\left(\overline{\mathcal{H}}_{\mathcal{N}_{t}^{2 \varepsilon}}-\overline{\mathcal{H}}_{\mathcal{N}_{t}^{2 \varepsilon}-1}\right)} \leq \alpha_{t}^{ \pm}, \max _{1 \leq j \leq \mathcal{N}_{t}^{2 \varepsilon}-1} \frac{\ell_{j}}{t} \leq \alpha_{t}^{ \pm}\right]
$$

and with $\overline{\mathcal{H}}_{k}:=Y_{2}^{t}\left(k e^{-\kappa \phi(t)}\right)=\frac{1}{t} \sum_{i=1}^{k} \mathcal{H}_{i}, \quad \bar{\ell}_{k}:=Y_{1}^{t}\left(k e^{-\kappa \phi(t)}\right)=\frac{1}{t} \sum_{i=1}^{k} \ell_{i}$ for any $k \in \mathbb{N}, \mathcal{N}_{t}^{2 \varepsilon}:=\inf \left\{m \geq 1, \overline{\mathcal{H}}_{m}>1-2 \varepsilon\right\}, \quad \alpha_{t}^{ \pm}:=\alpha\left(1 \pm(\log \log t)^{-1 / 2}\right)$, and $v$ is a positive function such that $\lim _{t \rightarrow+\infty} v(\varepsilon, t) \leq$ const $\times \varepsilon^{\kappa \wedge(1-\kappa)}$.

The proof of this proposition relies on the three following lemmata. The first one deals with the local time at the $h_{t}$-minima for which the diffusion $X$ already escaped before time $t$. The second deals with the local time at the last $h_{t}$-minimum $m_{N_{t}}$ in the remaining time before time $t$. Finally the last one is a technical point.

Lemma 5.2. For any large $t>0,2 \leq k \leq n_{t}$, any $x>0$ and $\gamma>0$ possibly depending on $t$, define the repartition function

$$
F_{\gamma}(x):=\mathbb{P}\left(\max _{1 \leq j \leq k-1} \mathcal{L}\left(H\left(\tilde{L}_{j}\right), \tilde{m}_{j}\right) \leq \gamma t, \sum_{i=1}^{k-1} U_{i} \leq x t\right) .
$$

Then for large $t$, for all $2 \leq k \leq n_{t}, x>0$ and $\gamma>0$,

$$
F_{\gamma}^{-}(x)-e^{-D_{1} h_{t}} \leq F_{\gamma}(x) \leq F_{\gamma}^{+}(x)+e^{-D_{1} h_{t}},
$$

where $F_{\gamma}^{ \pm}(x):=\mathbb{P}\left(\max _{1 \leq j \leq k-1} \ell_{j} \leq \gamma_{t}^{ \pm} t, \sum_{i=1}^{k-1} \mathcal{H}_{i} \leq x_{t}^{ \pm} t\right)$ with $\gamma_{t}^{ \pm}:=\gamma\left(1 \pm 2 \varepsilon_{t}\right)$, $x_{t}^{ \pm}:=x\left(1 \pm 2 \varepsilon_{t}\right), \varepsilon_{t}$ and $D_{1}$ are given in Proposition 3.5 .

Lemma 5.3. For any $t>0$, define for every $\gamma>0$ and $0<x<1$ possibly depending on $t$,

$f_{\gamma}(x):=E\left[\mathbb{P}_{\tilde{m}_{1}}^{W_{\kappa}}\left(\mathcal{L}_{X^{\prime}}\left(t(1-x), \tilde{m}_{1}\right) \leq \gamma t, H^{\prime}\left(\tilde{L}_{1}\right)>t(1-x), H^{\prime}\left(\tilde{L}_{1}\right)<H^{\prime}\left(\tilde{L}_{1}^{-}\right)\right)\right]$.

For such $t, \gamma$ and $x$, we also introduce

$\tilde{f}_{\gamma}(x)$

$:=E\left(\mathbb{P}_{\tilde{m}_{1}}^{W_{\kappa}}\left(\sup _{y \in \mathcal{D}_{1}} \mathcal{L}_{X^{\prime}}[t(1-x), y] \leq \gamma t, H^{\prime}\left(\tilde{L}_{1}\right)>t(1-x), H^{\prime}\left(\tilde{L}_{1}\right)<H^{\prime}\left(\tilde{L}_{1}^{-}\right)\right)\right)$,

with $\mathcal{D}_{1}$ defined in (3.13). Here $X^{\prime}$ is an independent copy of $X$ starting at $\tilde{m}_{1}$, and the definition of $H^{\prime}$ for $X^{\prime}$ is the same as the definition of $H$ for $X$. Let $\varepsilon \in(0,1 / 2)$. There exists $c_{2}>0$ such that for large $t$, for every $x \in[\varepsilon, 1-\varepsilon]$,

$$
f_{\gamma}^{-}(x)-o\left(n_{t}^{-1}\right) \leq \tilde{f}_{\gamma}(x) \leq f_{\gamma}(x) \leq f_{\gamma}^{+}(x)+o\left(n_{t}^{-1}\right),
$$

with $f_{\gamma}^{ \pm}(x):=\mathbb{P}\left(\frac{1}{R_{1}} \leq \frac{\gamma}{1-x}\left(1 \pm \varepsilon_{t}^{\prime}\right), \mathcal{H}_{1}>t(1-x)\left(1 \mp \varepsilon_{t}^{\prime}\right)\right)$ and $\varepsilon_{t}^{\prime}=e^{-c_{2} h_{t}}$. 
Lemma 5.4. For any $0<a<1 / 4$, we have for any $t>0$,

$$
\sum_{1 \leq k \leq n_{t}} \mathbb{P}\left[\overline{\mathcal{H}}_{k}>1-a / 2,1-2 a<\overline{\mathcal{H}}_{k-1} \leq 1-3 a / 4\right] \leq s(a, t),
$$

with $s(a, t)$ such that $\lim _{t \rightarrow+\infty} s(a, t)=$ const $\times a^{1-\kappa}$. For any $\varepsilon \in(0,1 / 2)$,

$$
\forall t>0, \quad \mathbb{P}\left[\varepsilon t \leq H\left(m_{N_{t}}\right) \leq(1-\varepsilon) t\right] \geq 1-\tilde{s}(\varepsilon, t),
$$

with $\tilde{s}(\varepsilon, t)$ such that $\lim _{t \rightarrow+\infty} \tilde{s}(\varepsilon, t)=$ const $\times \varepsilon^{(1-\kappa) \wedge \kappa}$.

We postpone the proof of these lemmata after the proof of Proposition 5.1.

Proof of Proposition 5.1: Recall from (1.3) that $N_{t}$ is the largest index such that $\sup _{s \leq t} X(s) \geq m_{N_{t}}$. In particular, $H\left(\tilde{L}_{j}\right) \leq H\left(\tilde{m}_{j+1}\right)=H\left(m_{j+1}\right) \leq H\left(m_{N_{t}}\right) \leq t$ for every $1 \leq j \leq N_{t}-1$ on $\mathcal{V}_{t} \cap\left\{N_{t} \leq n_{t}\right\}$. The main idea is to use the fact that the supremum of the local time at time $t$ is achieved in the neighborhood of the $h_{t}$-minima $m_{i}, 1 \leq i \leq n_{t}$.

We start with the upper bound. Let $\alpha>0$ and $0<\varepsilon<1 / 2$. Notice that $\mathbb{P}\left(N_{t}=0, \mathcal{V}_{t}\right) \leq \mathbb{P}\left[H\left(\tilde{m}_{1}\right)>t\right] \leq C_{2} v_{t}$ by (3.1). Using (3.1), (3.2), (3.3), (5.3), Lemma 2.2 and Remark 2.3, we have for $t$ large enough,

$$
\begin{aligned}
& \mathbb{P}\left(\sup _{x \in \mathbb{R}} \mathcal{L}(t, x) \leq \alpha t\right) \leq E\left[\mathbb{P}^{W_{\kappa}}\left(\max _{1 \leq j \leq N_{t}} \mathcal{L}\left(t, m_{j}\right) \leq \alpha t\right)\right] \\
\leq & E\left[\mathbb{P}^{W_{\kappa}}\left(\max _{1 \leq j \leq N_{t}-1} \mathcal{L}\left[H\left(\tilde{L}_{j}\right), \tilde{m}_{j}\right] \leq \alpha t, \mathcal{L}\left(t, \tilde{m}_{N_{t}}\right) \leq \alpha t, \mathcal{Q}, \mathcal{B}_{1}\left(n_{t}\right), \mathcal{B}_{2}\left(n_{t}\right), \mathcal{V}_{t}\right)\right] \\
& +\bar{s}(\varepsilon, t) .
\end{aligned}
$$

with $\mathcal{Q}:=\left\{\varepsilon t \leq H\left(m_{N_{t}}\right) \leq(1-\varepsilon) t, 1 \leq N_{t} \leq n_{t}\right\}$ and $\bar{s}$ satisfying $\lim _{t \rightarrow+\infty} \bar{s}(\varepsilon, t) \leq C_{+} \varepsilon^{(1-\kappa) \wedge \kappa}$. We will introduce in what follows different measures denoted by the letter $\nu$; they depend on $k$ but we do not write $k$ as a subscript to simplify the notation. First, define two measures $\nu_{1}^{W_{\kappa}}$ and $\nu_{2}^{W_{\kappa}}$ on $(0,1)$ by, for every $0<y<1$,

$$
\begin{aligned}
\nu_{1}^{W_{\kappa}}(y) & :=\nu_{1}^{W_{\kappa}}([0, y]) \\
& :=\mathbb{P}^{W_{\kappa}}\left(\max _{1 \leq j \leq k-1} \mathcal{L}\left[H\left(\tilde{L}_{j}\right), \tilde{m}_{j}\right] \leq \alpha t, H\left(\tilde{m}_{k}\right)-\sum_{i=1}^{k-1} U_{i}<\tilde{v}_{t}, H\left(\tilde{m}_{k}\right) \leq y t\right), \\
\nu_{2}^{W_{\kappa}}(y) & :=\nu_{2}^{W_{\kappa}}([0, y]) \\
& :=\mathbb{P}_{\tilde{m}_{k}}^{W_{\kappa}}\left(\mathcal{L}_{X^{\prime}}\left[t(1-y), \tilde{m}_{k}\right] \leq \alpha t, H^{\prime}\left(\tilde{m}_{k+1}\right)>t(1-y),\right. \\
& \left.H^{\prime}\left(\tilde{m}_{k+1}\right)<H^{\prime}\left(\tilde{L}_{k}^{-}\right), H^{\prime}\left(\tilde{m}_{k+1}\right)-H^{\prime}\left(\tilde{L}_{k}\right) \leq \tilde{v}_{t}\right),
\end{aligned}
$$

with $X^{\prime}$ a diffusion starting from $\tilde{m}_{k}$ independent of $X$ (conditionally on $W_{\kappa}$ ), and $H^{\prime}$ has the same definition as $H$ (see (1.1)) but for $X^{\prime}$. Partitioning on the values of $N_{t}$, and $H\left(\tilde{m}_{k}\right)$, we obtain by the strong markov property (applied at time $H\left(\tilde{m}_{k}\right)$ under $\left.\mathbb{P}^{W_{\kappa}}\right)$, that the probability $E\left[\mathbb{P}^{W_{\kappa}}().\right]$ in the line below (5.4) is smaller than

$$
\sum_{1 \leq k \leq n_{t}} \int_{\varepsilon}^{1-\varepsilon} E\left(\nu_{2}^{W_{\kappa}}(x) \mathrm{d} \nu_{1}^{W_{\kappa}}(x)\right)=\sum_{1 \leq k \leq n_{t}} E\left[\int_{\varepsilon}^{1-\varepsilon} \nu_{2}^{W_{\kappa}}(x) \mathrm{d} \nu_{1}^{W_{\kappa}}(x)\right] .
$$


The next step is to prove that the previous expectation can be approximated by a product of expectations. First notice that both $y \rightarrow \nu_{1}^{W_{\kappa}}(y)$ and $y \rightarrow \nu_{2}^{W_{\kappa}}(y)$ are positive increasing. So integrating by parts

$$
\begin{aligned}
\int_{\varepsilon}^{1-\varepsilon} \nu_{2}^{W_{\kappa}}(x) \mathrm{d} \nu_{1}^{W_{\kappa}}(x) & =\left[\nu_{2}^{W_{\kappa}}(x) \nu_{1}^{W_{\kappa}}(x)\right]_{\varepsilon}^{1-\varepsilon}-\int_{\varepsilon}^{1-\varepsilon} \nu_{1}^{W_{\kappa}}(x) \mathrm{d} \nu_{2}^{W_{\kappa}}(x) \\
& \leq\left[\nu_{2}^{W_{\kappa}}(x) \nu_{1}^{W_{\kappa}}(x)\right]_{\varepsilon}^{1-\varepsilon}-\int_{\varepsilon}^{1-\varepsilon} \widetilde{\nu}_{1}^{W_{\kappa}}(x) \mathrm{d} \nu_{2}^{W_{\kappa}}(x) \\
& =\left[\nu_{2}^{W_{\kappa}}(x)\left(\nu_{1}^{W_{\kappa}}(x)-\widetilde{\nu}_{1}^{W_{\kappa}}(x)\right)\right]_{\varepsilon}^{1-\varepsilon}+\mathcal{I}_{1},
\end{aligned}
$$

with $\widetilde{\nu}_{1}^{W_{\kappa}}(x):=\mathbb{P}^{W_{\kappa}}\left(\mathcal{G}_{1}, H\left(\tilde{m}_{k}\right)-\sum_{i=1}^{k-1} U_{i}<\tilde{v}_{t}, \sum_{i=1}^{k-1} U_{i}+\tilde{v}_{t} \leq x t\right) \leq \nu_{1}^{W_{\kappa}}(x)$ and $\mathcal{G}_{1}:=\left\{\max _{1 \leq j \leq k-1} \mathcal{L}\left(H\left(\tilde{L}_{j}\right), \tilde{m}_{j}\right) \leq \alpha t\right\}$ and

$$
\begin{aligned}
\mathcal{I}_{1} & :=\int_{\varepsilon}^{1-\varepsilon} \nu_{2}^{W_{\kappa}}(x) \mathrm{d} \tilde{\nu}_{1}(x) \leq \int_{\varepsilon}^{1-\varepsilon} \nu_{2}^{W_{\kappa}}(x) \mathrm{d} \nu_{3}^{W_{\kappa}}(x)=: \mathcal{I}_{1}^{\prime}, \\
\nu_{3}^{W_{\kappa}}(x) & :=\mathbb{P}^{W_{\kappa}}\left(\mathcal{G}_{1}, \sum_{i=1}^{k-1} U_{i}+\tilde{v}_{t} \leq x t\right) .
\end{aligned}
$$

First, we deal with what is going to be a negligible part, that is to say the first term in (5.6). As $\nu_{1}^{W_{\kappa}}(x) \leq \mathbb{P}^{W_{\kappa}}\left(\mathcal{G}_{1}, H\left(\tilde{m}_{k}\right)-\sum_{i=1}^{k-1} U_{i}<\tilde{v}_{t}, \sum_{i=1}^{k-1} U_{i} \leq x t\right)$ because by definition $\sum_{i=1}^{k-1} U_{i}<H\left(\tilde{m}_{k}\right)$, we have, for $\varepsilon<x<1-\varepsilon$,

$$
\left|\nu_{1}^{W_{\kappa}}(x)-\widetilde{\nu}_{1}^{W_{\kappa}}(x)\right| \leq \mathbb{P}^{W_{\kappa}}\left(x t-\widetilde{v}_{t}<\sum_{i=1}^{k-1} U_{i} \leq x t\right)=: h_{k}(x) .
$$

so $\left[\nu_{2}^{W_{\kappa}}(x)\left(\nu_{1}^{W_{\kappa}}(x)-\widetilde{\nu}_{1}^{W_{\kappa}}(x)\right)\right]_{\varepsilon}^{1-\varepsilon} \leq \nu_{2}^{W_{\kappa}}(1-\varepsilon) h_{k}(1-\varepsilon)+\nu_{2}^{W_{\kappa}}(\varepsilon) h_{k}(\varepsilon)$. Notice that $\sum_{i=1}^{k-1} U_{i}$ is measurable with respect to $\sigma\left(X(s), 0 \leq s \leq H\left(\tilde{L}_{k-1}\right) ; W_{\kappa}(x), x \leq\right.$ $\left.\tilde{L}_{k-1}^{+}\right)$, since $\tilde{L}_{k-1} \leq \tilde{L}_{k-1}^{+}$, whereas the event in the definition of $\nu_{2}^{W_{\kappa}}$ belongs to $\sigma\left(X^{\prime}(s), 0 \leq s \leq \min \left(H^{\prime}\left(\tilde{L}_{k}^{-}\right), H^{\prime}\left(\tilde{m}_{k+1}\right)\right) ; W_{\kappa}(x)-W_{\kappa}\left(\tilde{m}_{k}\right), \tilde{L}_{k-1}^{+} \leq x \leq \tilde{L}_{k+1}^{+}\right)$, with $X^{\prime}$ an independent copy of $X$ starting at $\tilde{m}_{k}$.

So independence of $X$ and $X^{\prime}$, and independence of the two portions of the environment involved (see Lemma 2.2) imply independence between $\nu_{2}^{W_{\kappa}}$ and $h_{k}$. Hence,

$$
\begin{aligned}
& E\left(\left[\nu_{2}^{W_{\kappa}}(x)\left(\nu_{1}^{W_{\kappa}}(x)-\widetilde{\nu}_{1}^{W_{\kappa}}(x)\right)\right]_{\varepsilon}^{1-\varepsilon}\right) \\
\leq & E\left[\nu_{2}^{W_{\kappa}}(1-\varepsilon)\right] E\left[h_{k}(1-\varepsilon)\right]+E\left[\nu_{2}^{W_{\kappa}}(\varepsilon)\right] E\left[h_{k}(\varepsilon)\right] \\
= & E\left[\widetilde{\nu}_{2}^{W_{\kappa}}(1-\varepsilon)\right] E\left[h_{k}(1-\varepsilon)\right]+E\left[\widetilde{\nu}_{2}^{W_{\kappa}}(\varepsilon)\right] E\left[h_{k}(\varepsilon)\right] .
\end{aligned}
$$

with for any $x$,

$$
\begin{aligned}
\widetilde{\nu}_{2}^{W_{\kappa}}(x):=\mathbb{P}_{\tilde{m}_{1}}^{W_{\kappa}}\left(\mathcal{L}_{X}\left[t(1-x), \tilde{m}_{1}\right] \leq \alpha t, H\left(\tilde{m}_{2}\right)>t(1-x)\right. \\
\left.H\left(\tilde{m}_{2}\right)<H\left(\tilde{L}_{1}^{-}\right), H\left(\tilde{m}_{2}\right)-H\left(\tilde{L}_{1}\right) \leq \tilde{v}_{t}\right)
\end{aligned}
$$


As $E\left(\widetilde{\nu}_{2}^{W_{\kappa}}(x)\right) \leq \mathbb{P}\left[U_{1}>t(1-x)-\tilde{v}_{t}\right]$ and for every small $\varepsilon>0$ and $t$ large enough $h_{k}(x) \leq \mathbb{P}^{W_{\kappa}}\left((x-\varepsilon) t<\sum_{i=1}^{k-1} U_{i} \leq x t\right)$ we can apply Proposition 3.5, we get

$$
\begin{aligned}
& E\left[h_{k}(1-\varepsilon)\right] E\left[\tilde{\nu}_{2}^{W_{\kappa}}(1-\varepsilon)\right] \\
\leq & \mathbb{P}\left(\frac{1-2 \varepsilon}{1+\varepsilon_{t}}<\sum_{i=1}^{k-1} \frac{\mathcal{H}_{i}}{t} \leq \frac{1-\varepsilon}{1-\varepsilon_{t}}\right) \mathbb{P}\left(\mathcal{H}_{1}>\frac{t \varepsilon-\tilde{v}_{t}}{1+\varepsilon_{t}}\right)+3 e^{-D_{1} h_{t}} .
\end{aligned}
$$

By (4.2) and the first part of Lemma 6.2, for any $0<a<1$ and $b>0$,

$$
\begin{aligned}
\lim _{t \rightarrow+\infty} \sum_{1 \leq k \leq n_{t}} \mathbb{P}\left(1-a<\sum_{i=1}^{k-1} \frac{\mathcal{H}_{i}}{t} \leq 1\right) \mathbb{P}\left(\mathcal{H}_{1}>t b\right) & =\frac{\text { const }}{b^{\kappa}} \int_{1-a}^{1} y^{\kappa-1} \mathrm{~d} y \\
& \leq \frac{\text { const }}{b^{\kappa}}\left(1-(1-a)^{\kappa}\right) .
\end{aligned}
$$

Therefore, we obtain

$$
\sum_{1 \leq k \leq n_{t}} E\left[\tilde{\nu}_{2}^{W_{\kappa}}(1-\varepsilon)\right] E\left[h_{k}(1-\varepsilon)\right] \leq C_{+} \cdot u(t, \varepsilon)
$$

with $u$ a positive function such that $\lim _{t \rightarrow+\infty} u(t, \varepsilon)=\max \left(\varepsilon^{1-\kappa}, \varepsilon^{\kappa}\right)$. A similar argument also works for the second term in (5.7), which yields

$$
\sum_{1 \leq k \leq n_{t}} E\left[\left[\nu_{2}^{W_{\kappa}}(x)\left(\nu_{1}^{W_{\kappa}}(x)-\tilde{\nu}_{1}^{W_{\kappa}}(x)\right)\right]_{\varepsilon}^{1-\varepsilon}\right] \leq 2 C_{+} \cdot u(t, \varepsilon) .
$$

We now deal with $\mathcal{I}_{1}^{\prime}$. By independence between $X$ and $X^{\prime}$, and the independent parts of the potential $W_{\kappa}$ involved in $\nu_{2}^{W_{\kappa}}(x)$ and $\nu_{3}^{W_{\kappa}}(x)$,

$$
E\left(\mathcal{I}_{1}^{\prime}\right)=\int_{\varepsilon}^{1-\varepsilon} \nu_{2}(x) \mathrm{d} \nu_{3}(x)
$$

with $\nu_{2}(x):=E\left(\nu_{2}^{W_{\kappa}}(x)\right)=E\left(\widetilde{\nu}_{2}^{W_{\kappa}}(x)\right)$ and $\nu_{3}(x):=E\left(\nu_{3}^{W_{\kappa}}(x)\right)$.

By the lower bound in Lemma 5.2, we have $\nu_{3}(x)=F_{\alpha}\left(x-\tilde{v}_{t} / t\right) \geq F_{\alpha}^{-}(x-$ $\left.\tilde{v}_{t} / t\right)-e^{-D_{1} h_{t}}$ for every $x>\varepsilon$ for large $t$. So, again since $y \rightarrow \nu_{2}(y)$ is positive increasing and $\nu_{3}$ is a repartition function, integrating by parts twice as in (5.6) gives with the change of variables $u=x-\tilde{v}_{t} / t$,

$$
\begin{aligned}
\int_{\varepsilon}^{1-\varepsilon} \nu_{2}(x) \mathrm{d} \nu_{3}(x) \leq & \int_{\varepsilon-\tilde{v}_{t} / t}^{1-\varepsilon-\tilde{v}_{t} / t} \nu_{2}\left(x+\tilde{v}_{t} / t\right) \mathrm{d} F_{\alpha}^{-}(x)+e^{-D_{1} h_{t}} \\
& +\left[\left(F_{\alpha}(x)-F_{\alpha}^{-}(x)\right) \nu_{2}\left(x+\tilde{v}_{t} / t\right)\right]_{\varepsilon-\tilde{v}_{t} / t}^{1-\varepsilon-\tilde{v}_{t} / t} .
\end{aligned}
$$

Recall (see before Lemma 3.1) that $\tilde{v}_{t} / t=2 / \log \left(h_{t}\right)=o(1)$ as $t \rightarrow+\infty$. Then we can prove in a similar way we have obtained (5.9) that:

$$
\sum_{1 \leq k \leq n_{t}}\left[\left(F_{\alpha}(x)-F_{\alpha}^{-}(x)\right) \nu_{2}\left(x+\tilde{v}_{t} / t\right)\right]_{\varepsilon-\tilde{v}_{t} / t}^{1-\varepsilon-\tilde{v}_{t} / t} \leq C_{+} \cdot u(t, \varepsilon)
$$

with as usual a possibly enlarged $C_{+}$. Indeed by Lemma $5.2,-\left(F_{\alpha}\left(\varepsilon-\tilde{v}_{t} / t\right)-\right.$ $\left.F_{\alpha}^{-}\left(\varepsilon-\tilde{v}_{t} / t\right)\right) \nu_{2}(\varepsilon) \leq e^{-D_{1} t}=o\left(n_{t}^{-1}\right)$ for every $1 \leq k \leq n_{t}$ for large $t$, and $\left(F_{\alpha}-F_{\alpha}^{-}\right)\left(1-\varepsilon-\tilde{v}_{t} / t\right) \leq\left(F_{\alpha}^{+}-F_{\alpha}^{-}\right)\left(1-\varepsilon-\tilde{v}_{t} / t\right)+e^{-D_{1} t} \leq \mathbb{P}\left(\max _{1 \leq j \leq k-1} \ell_{j} \in\right.$ $\left.\left[\gamma_{t}^{-} t, \gamma_{t}^{+} t\right]\right)+\mathbb{P}\left(\sum_{i=1}^{k-1} \mathcal{H}_{i} \in\left[x_{t}^{-} t, x_{t}^{+} t\right]\right)+e^{-D_{1} t}$ for every $k \leq n_{t}$ for large $t$, with $\gamma=$ 
$\alpha$ and $x=1-\varepsilon-\tilde{v}_{t} / t$. The first probability is less than $n_{t} \mathbb{P}\left(S_{1} \mathbf{e}_{1} \in\left[\gamma_{t}^{-} t, \gamma_{t}^{+} t\right]\right)=$ $n_{t} \mathbb{E}\left(\int_{\gamma t\left(1-2 \varepsilon_{t}\right) / S_{1}}^{\gamma t\left(1+2 \varepsilon_{t}\right) / S_{1}} e^{-u / 2} \mathrm{~d} u / 2\right) \leq 8 n_{t} \varepsilon_{t} \sup _{v \geq 0}\left(v e^{-v}\right)=o\left(1 / n_{t}\right)$, whereas the second one is treated as (5.8), which leads to (5.12).

So the important term in the right hand side of inequality (5.11) comes from the integral. We now work on $\nu_{2}(x)$. We have,

$$
\begin{aligned}
& \nu_{2}(x) \\
& \leq E\left(\mathbb{P}_{\tilde{m}_{1}}^{W_{\kappa}}\left[\mathcal{L}_{X}\left(t(1-x), \tilde{m}_{1}\right) \leq \alpha t, H\left(\tilde{L}_{1}\right)>t(1-x)-\tilde{v}_{t}, H\left(\tilde{L}_{1}\right)<H\left(\tilde{L}_{1}^{-}\right)\right]\right) \\
& \leq E\left(\mathbb { P } _ { \tilde { m } _ { 1 } } ^ { W _ { \kappa } } \left[\mathcal{L}_{X}\left(t(1-x)-\tilde{v}_{t}, \tilde{m}_{1}\right) \leq \alpha t,\right.\right. \\
& \left.\left.\quad H\left(\tilde{L}_{1}\right)>t(1-x)-\tilde{v}_{t}, H\left(\tilde{L}_{1}\right)<H\left(\tilde{L}_{1}^{-}\right)\right]\right)=f_{\alpha}\left(x+\tilde{v}_{t} / t\right),
\end{aligned}
$$

as defined in Lemma 5.3. Then, as $F_{\alpha}^{-}(x)$ is positive and increasing in $x$, using Lemma 5.3 with $\gamma=\alpha$, we obtain

$$
\int_{\varepsilon-\tilde{v}_{t} / t}^{1-\varepsilon-\tilde{v}_{t} / t} \nu_{2}\left(x+\tilde{v}_{t} / t\right) \mathrm{d} F_{\alpha}^{-}(x) \leq \int_{\varepsilon-\tilde{v}_{t} / t}^{1-\varepsilon-\tilde{v}_{t} / t} f_{\alpha}^{+}\left(x+2 \tilde{v}_{t} / t\right) \mathrm{d} F_{\alpha}^{-}(x)+o\left(n_{t}^{-1}\right) .
$$

Now, as $f_{\alpha}^{+}\left(x+2 \tilde{v}_{t} / t\right)$ can be written (since $\mathcal{H}_{k}=\ell_{k} R_{k}$, see Proposition 3.5),

$f_{\alpha}^{+}\left(x+2 \tilde{v}_{t} / t\right)=\mathbb{P}\left(\left(1-x-2 \tilde{v}_{t} / t\right) \frac{\ell_{k}}{\mathcal{H}_{k}} \leq \alpha\left(1+\varepsilon_{t}^{\prime}\right), \mathcal{H}_{k}>t\left(1-x-2 \tilde{v}_{t} / t\right)\left(1-\varepsilon_{t}^{\prime}\right)\right)$, we get by independence of the random variables $\left(\left(\ell_{j}, \mathcal{H}_{j}\right), j \leq n_{t}\right)$,

$$
\begin{aligned}
& \int_{\varepsilon-\tilde{v}_{t} / t}^{1-\varepsilon-\tilde{v}_{t} / t} f_{\alpha}^{+}\left(x+2 \tilde{v}_{t} / t\right) \mathrm{d} F_{\alpha}^{-}(x) \\
& \leq \mathbb{P}\left[\left(1-\overline{\mathcal{H}}_{k-1}\right) \frac{\bar{\ell}_{k}-\bar{\ell}_{k-1}}{\overline{\mathcal{H}}_{k}-\overline{\mathcal{H}}_{k-1}} \leq \alpha+\tilde{\varepsilon}_{t}(k), \overline{\mathcal{H}}_{k} \geq 1-\delta_{t}^{\prime},\right. \\
&\left.\max _{1 \leq j \leq k-1} \frac{\ell_{j}}{t} \leq \alpha, \overline{\mathcal{H}}_{k-1} \leq 1-\varepsilon+\delta_{t}^{\prime}\right],
\end{aligned}
$$

with $\delta_{t}^{\prime}:=3 \tilde{v}_{t} / t, \tilde{\varepsilon}_{t}(k):=\left(\alpha+\ell_{k} / \mathcal{H}_{k}\right) \delta_{t}^{\prime}$.

The idea now is to make appear the event $\left\{\mathcal{N}_{t}^{2 \varepsilon}=k\right\}$ in the above probability (recall the definition of $\mathcal{N}_{t}^{2 \varepsilon}$ given in Proposition 5.1) and then sum over $k$.

We first prove that the sum over $k \leq n_{t}$, of the above probability is small if we intersect its event with the event $\left\{\mathcal{N}_{t}^{2 \varepsilon} \neq k\right\}$. In other words, let us prove that

$$
\sum_{1}:=\sum_{1 \leq k \leq n_{t}} \mathbb{P}\left[\overline{\mathcal{H}}_{k} \geq 1-\delta_{t}^{\prime}, \overline{\mathcal{H}}_{k-1} \leq 1-\varepsilon+\delta_{t}^{\prime}, \mathcal{N}_{t}^{2 \varepsilon} \neq k\right]
$$

is small. As $\left\{\mathcal{N}_{t}^{2 \varepsilon} \neq k\right\}=\left\{\overline{\mathcal{H}}_{k} \leq 1-2 \varepsilon\right\} \cup\left\{\overline{\mathcal{H}}_{k-1}>1-2 \varepsilon\right\}$, and since for $t$ large enough, $\left\{\overline{\mathcal{H}}_{k} \geq 1-\delta_{t}^{\prime}\right\} \cap\left\{\overline{\mathcal{H}}_{k} \leq 1-2 \varepsilon\right\}=\emptyset$, we have

$$
\sum_{1} \leq \sum_{1 \leq k \leq n_{t}} \mathbb{P}\left[\overline{\mathcal{H}}_{k} \geq 1-\delta_{t}^{\prime}, 1-2 \varepsilon<\overline{\mathcal{H}}_{k-1} \leq 1-\varepsilon+\delta_{t}^{\prime}\right]
$$

Therefore, for $t$ large enough, with $s(\varepsilon, t)$ defined in Lemma 5.4,

$$
\sum_{1} \leq \sum_{1 \leq k \leq n_{t}} \mathbb{P}\left[\overline{\mathcal{H}}_{k}>1-\varepsilon / 2,1-2 \varepsilon<\overline{\mathcal{H}}_{k-1} \leq 1-3 \varepsilon / 4\right] \leq s(\varepsilon, t)
$$


by (5.2). Finally, combining equations from (5.10) to (5.16) leads to

$$
\begin{aligned}
& \sum_{1 \leq k \leq n_{t}} E\left(\mathcal{I}_{1}^{\prime}\right) \\
\leq & \mathbb{P}\left[\left(1-\overline{\mathcal{H}}_{\mathcal{N}_{t}^{2 \varepsilon}-1}\right) \frac{\bar{\ell}_{\mathcal{N}_{t}^{2 \varepsilon}}-\bar{\ell}_{\mathcal{N}_{t}^{2 \varepsilon}-1}}{\overline{\mathcal{H}}_{\mathcal{N}_{t}^{2 \varepsilon}}-\overline{\mathcal{H}}_{\mathcal{N}_{t}^{2 \varepsilon}-1}} \leq \alpha+\tilde{\varepsilon}_{t}\left(\mathcal{N}_{t}^{2 \varepsilon}\right), \max _{1 \leq j \leq \mathcal{N}_{t}^{2 \varepsilon}-1} \frac{\ell_{j}}{t} \leq \alpha\right] \\
& +s(\varepsilon, t)+C_{+} u(t, \varepsilon)+o(1) .
\end{aligned}
$$

To finish we have to deal with $\tilde{\varepsilon}_{t}\left(\mathcal{N}_{t}^{2 \varepsilon}\right)$, a basic computation partitioning on the values of $\mathcal{N}_{t}^{2 \varepsilon}$, shows that $\left.\mathbb{P}\left[\tilde{\varepsilon}_{t}\left(\mathcal{N}_{t}^{2 \varepsilon}\right)\right) \geq \alpha \sqrt{\delta_{t}^{\prime}} / 6\right] \leq C_{+} \mathbb{P}\left(R_{1} \leq \sqrt{\delta}_{t}^{\prime}\right)=o(1)$ as $R_{1}$ converges in distribution to $\mathcal{R}_{\kappa}$ which is almost surely positive. Collecting this last fact, (5.4), (5.5), (5.6), (5.9) and (5.17) finish the proof of the upper bound.

\section{Proof of the lower bound:}

The proof here follows the same line as the upper bound. The main difference comes from the fact that we can no longer use the inequality $\sup _{x \in \mathbb{R}} \mathcal{L}(t, x) \geq$ $\sup _{1 \leq j \leq N_{t}} \mathcal{L}\left(t, m_{j}\right)$. So for this part of the proof we stress on what is different from the upper bound, and refer to the previous computations when very few changes occur.

Assume for the moment that

$$
\mathbb{P}\left(\left\{\sup _{x \in \mathbb{R}} \mathcal{L}(t, x) \geq 2 \tilde{w}_{t}\right\}=: \mathcal{E}_{2}\right) \geq 1-o(1),
$$

with $\tilde{w}_{t}:=t e^{(\kappa(1+3 \delta)-1) \phi(t)}$, and recall that $\delta$ is chosen small enough such that $\kappa(1+3 \delta)<1$ (see Lemma 3.2). This fact (5.18) is a direct consequence of the upperbound of $\mathbb{P}\left(\sup _{x \in \mathbb{R}} \mathcal{L}(t, x) \leq \alpha t\right)$ (see at the beginning of the proof of Theorem 1.3 page 908 for a proof of (5.18)). Recall (3.13), and define for any $\ell \geq 1$,

$$
\begin{aligned}
& \mathcal{E}_{3}(\ell):=\mathcal{E}_{3}^{1}(\ell) \cap \mathcal{E}_{3}^{2}(\ell), \quad \text { with } \\
& \mathcal{E}_{3}^{1}(\ell):=\bigcap_{j=1}^{\ell-1}\left\{\sup _{x \in \mathcal{D}_{j}}\left[\mathcal{L}\left(H\left(\tilde{L}_{j}\right), x\right)-\mathcal{L}\left(H\left(\tilde{m}_{j}\right), x\right)\right] \leq t \tilde{\alpha}_{t}\right\}, \\
& \mathcal{E}_{3}^{2}(\ell):=\left\{\sup _{x \in \mathcal{D}_{\ell}}\left[\mathcal{L}(t, x)-\mathcal{L}\left(H\left(\tilde{m}_{\ell}\right), x\right)\right] \leq t \tilde{\alpha}_{t}\right\},
\end{aligned}
$$

with $\tilde{\alpha}_{t}:=\left(\alpha t-2 \tilde{w}_{t}\right) / t$. Recall the definitions of the events $\mathcal{B}_{i}, 1 \leq i \leq 4$ in Sections 3.1 and 3.2. We have for large $t$,

$$
\begin{aligned}
& \left\{\sup _{x \in \mathbb{R}_{+}} \mathcal{L}(t, x) \leq \alpha t\right\} \cap \mathcal{V}_{t} \cap \mathcal{E}_{2} \cap\left\{N_{t} \leq n_{t}\right\} \cap \cap_{i=1}^{4} \mathcal{B}_{i}\left(n_{t}\right) \\
\supset & \mathcal{E}_{3}\left(N_{t}\right) \cap \mathcal{V}_{t} \cap \mathcal{E}_{2} \cap\left\{N_{t} \leq n_{t}\right\} \cap \cap_{i=1}^{4} \mathcal{B}_{i}\left(n_{t}\right) .
\end{aligned}
$$

Indeed, $\mathcal{L}(t, x) \leq \tilde{w}_{t}$ for every $x \in\left(\mathbb{R}_{+}-\cup_{j=1}^{n_{t}}\left[\tilde{L}_{j}^{-}, \tilde{L}_{j}\right]\right)$ on $\mathcal{B}_{2}\left(n_{t}\right) \cap \mathcal{B}_{3}\left(n_{t}\right) \cap \mathcal{V}_{t} \cap$ $\left\{N_{t} \leq n_{t}\right\}$, and on the same event intersected with $\mathcal{B}_{4}\left(n_{t}\right), \mathcal{L}(t, x) \leq \tilde{w}_{t}+t e^{-2 \phi(t)}<$ $2 \tilde{w}_{t}$ for every $x \in \cup_{j=1}^{n_{t}}\left(\left[\tilde{L}_{j}^{-}, \tilde{L}_{j}\right] \cap \overline{\mathcal{D}_{j}}\right)$, whereas for $x \in \mathcal{D}_{j}, \mathcal{L}\left(H\left(\tilde{m}_{j}\right), x\right) \leq \tilde{w}_{t}$ if $j \leq n_{t}$ and $\mathcal{L}(t, x)-\mathcal{L}\left(H\left(\tilde{L}_{j}\right), x\right) \leq \tilde{w}_{t}$ if $j<N_{t}$. Notice that by Lemmata 2.2, $3.1,3.2,3.4$ and the above assumption (5.18),

$$
\mathbb{P}\left(\mathcal{V}_{t} \cap \mathcal{E}_{2} \cap\left\{N_{t} \leq n_{t}\right\} \cap \cap_{i=1}^{4} \mathcal{B}_{i}\left(n_{t}\right)\right) \geq 1-o(1) .
$$

We now deal with $\mathbb{P}\left(\mathcal{E}_{3}\left(N_{t}\right) \cap \mathcal{B}_{1}\left(N_{t}\right) \cap \mathcal{B}_{2}\left(n_{t}\right) \cap \mathcal{V}_{t} \cap\left\{N_{t} \leq n_{t}\right\}\right)$. Using Lemma 2.2, the fact that $H\left(\tilde{L}_{k}\right) \leq H\left(\tilde{m}_{k+1}\right)$ and the strong Markov property with respect to 
$\mathbb{P}^{W_{\kappa}}$, we obtain

$$
\begin{aligned}
& \mathbb{P}\left(\mathcal{E}_{3}\left(N_{t}\right) \cap \mathcal{B}_{1}\left(N_{t}\right) \cap \mathcal{B}_{2}\left(n_{t}\right) \cap \mathcal{V}_{t} \cap \mathcal{Q}\right) \\
& \geq \sum_{k=1}^{n_{t}} E\left(\int_{\varepsilon}^{1-\varepsilon} \nu_{4}^{W_{\kappa}}(y) \mathbb{P}^{W_{\kappa}}\left(\mathcal{E}_{3}^{1}(k), \mathcal{B}_{1}(k), \mathcal{B}_{2}(k-1), H\left(\tilde{m}_{k}\right) / t \in \mathrm{d} y\right)\right)-o(1)
\end{aligned}
$$

with

$$
\begin{aligned}
& \nu_{4}^{W_{\kappa}}(y) \\
:= & \mathbb{P}_{\tilde{m}_{k}}^{W_{\kappa}}\left(\sup _{x \in \mathcal{D}_{k}} \mathcal{L}_{X}(t(1-y), x) \leq t \tilde{\alpha}_{t}, H\left(\tilde{L}_{k}\right)>t(1-y), H\left(\tilde{L}_{k}\right)<H\left(\tilde{L}_{k}^{-}\right)\right),
\end{aligned}
$$

Now, by computations similar to the ones giving the upper bounds in (5.9) and (5.10), we have

$$
\begin{aligned}
& \mathbb{P}\left(\mathcal{E}_{3}\left(N_{t}\right) \cap \mathcal{B}_{1}\left(N_{t}\right) \cap \mathcal{B}_{2}\left(n_{t}\right) \cap \mathcal{V}_{t}, \mathcal{Q}\right) \\
& \geq \sum_{k=1}^{n_{t}} \int_{\varepsilon}^{1-\varepsilon} E\left(\nu_{4}^{W_{\kappa}}(y) \mathrm{d} \nu_{5}^{W_{\kappa}}(y)\right)-o(1)=\sum_{k=1}^{n_{t}} \int_{\varepsilon}^{1-\varepsilon} \nu_{4}(y) \mathrm{d} \nu_{5}(y)-o(1) .
\end{aligned}
$$

with $\nu_{5}^{W_{\kappa}}(y):=\mathbb{P}^{W_{\kappa}}\left(\mathcal{E}_{3}^{1}(k), \mathcal{B}_{1}(k), \mathcal{B}_{2}(k-1), \sum_{i=1}^{k-1} U_{i} / t \leq y\right), \nu_{4}(y):=E\left(\nu_{4}^{W_{\kappa}}(y)\right)$ and $\nu_{5}(y):=E\left(\nu_{5}^{W_{\kappa}}(y)\right)$. The next step is to remove $\mathcal{B}_{1}(k)$ in the above expression. For that, we only have to prove that

$$
\sum_{k=1}^{n_{t}} \int_{\varepsilon}^{1-\varepsilon} E\left(\nu_{4}^{W_{\kappa}}(y) \mathbb{P}^{W_{\kappa}}\left(\mathcal{E}_{3}^{1}(k), \overline{\mathcal{B}}_{1}(k), \mathcal{B}_{2}(k-1), \sum_{i=1}^{k-1} U_{i} / t \in \mathrm{d} y\right)\right)
$$

is negligible, one can check that this quantity is smaller than

$$
\begin{aligned}
& \sum_{k=1}^{n_{t}} \int_{\varepsilon}^{1-\varepsilon} E\left[\mathbb{P}_{\tilde{m}_{k}}^{W_{\kappa}}\left(H\left(\tilde{L}_{k}\right)<H\left(\tilde{L}_{k}^{-}\right), H\left(\tilde{L}_{k}\right)>t(1-y)\right)\right] \\
& \qquad \mathbb{P}\left(\overline{\mathcal{B}}_{1}(k), \mathcal{B}_{2}(k-1), \sum_{i=1}^{k-1} U_{i} / t \in \mathrm{d} y\right) \\
& \leq \sum_{k=1}^{n_{t}} \mathbb{P}\left(\sum_{i=1}^{k-1} U_{i} / t \leq 1, \sum_{i=1}^{k} U_{i} / t>1, \overline{\mathcal{B}}_{1}(k)\right) \\
& \leq \mathbb{P}\left(\overline{\mathcal{B}}_{1}\left(n_{t}\right)\right) \leq C_{2} v_{t}=o(1),
\end{aligned}
$$

where the last inequality comes from (3.1). Therefore, collecting the above computations yields

$$
\mathbb{P}\left(\sup _{x \in \mathbb{R}} \mathcal{L}(t, x) \leq \alpha\right) \geq \sum_{k=1}^{n_{t}} \int_{\varepsilon}^{1-\varepsilon} \nu_{4}(y) \mathrm{d} \tilde{\nu}_{5}(y)-o(1),
$$

with $\tilde{\nu}_{5}(y):=e^{-\kappa \phi(t)} \mathbb{P}\left(\mathcal{E}_{3}^{1}(k), \mathcal{B}_{2}(k-1), \sum_{i=1}^{k-1} U_{i} / t \leq y\right)$.

We start with an estimation of the repartition function $\tilde{\nu}_{5}(y)$. Recall that like in the proof of Lemma 3.6, by the strong Markov property, the occupation time formula (1.12) and (1.13) the sequence $\left(U_{j},\left\{\mathcal{L}\left(H\left(\tilde{L}_{j}\right), x\right)-\mathcal{L}\left(H\left(\tilde{m}_{j}\right), x\right), x \in \mathcal{D}_{j}\right\}, j \leq\right.$ 
$\left.n_{t}\right)$ under $\mathcal{B}_{2}\left(n_{t}\right)$ is equal to a sequence $\left(H_{j}\left(\tilde{L}_{j}\right),\left\{\mathcal{L}_{j}\left(H_{j}\left(\tilde{L}_{j}\right), x\right), x \in \mathcal{D}_{j}\right\}, j \leq n_{t}\right)$, with this time

$$
\begin{aligned}
H_{j}\left(\tilde{L}_{j}\right) & :=A^{j}\left(\tilde{L}_{j}\right) \int_{\tilde{L}_{j}^{-}}^{\tilde{L}_{j}} e^{-\tilde{V}^{(j)}(u)} \mathcal{L}_{B^{j}}\left[\tau^{B^{j}}(1), A^{j}(u) / A^{j}\left(\tilde{L}_{j}\right)\right] \mathrm{d} u, \\
\mathcal{L}_{j}\left(H_{j}\left(\tilde{L}_{j}\right), x\right) & :=A^{j}\left(\tilde{L}_{j}\right) e^{-\tilde{V}^{(j)}(x)} \mathcal{L}_{B^{j}}\left[\tau^{B^{j}}(1), A^{j}(x) / A^{j}\left(\tilde{L}_{j}\right)\right],
\end{aligned}
$$

where $A^{j}(u)=\int_{\tilde{m}_{j}}^{u} e^{\tilde{V}^{(j)}(x)} \mathrm{d} x$. Using Remark 2.3, Lemma 2.2, Fact 2.1 (ii), and then (7.5) and (7.6), we have for large $t$ for any $1 \leq j \leq n_{t}$ since $\phi(t)=o(\log t)$,

$$
\begin{gathered}
P\left[\tilde{\tau}_{j}\left(\kappa r_{t} / 8\right) \leq \tilde{m}_{j}+r_{t} \leq \tilde{\tau}_{j}\left(r_{t}\right)\right] \geq 1-C_{+} e^{-\left(c_{-}\right) r_{t}}, \\
P\left[\tilde{\tau}_{j}^{-}\left(r_{t}\right) \leq \tilde{m}_{j}-r_{t} \leq \tilde{\tau}_{j}^{-}\left(\kappa r_{t} / 8\right)\right] \geq 1-C_{+} e^{-\left(c_{-}\right) r_{t}} .
\end{gathered}
$$

with $c_{-}>0$. Therefore for any $j, P\left(\mathcal{D}_{j} \subset\left[\tilde{\tau}_{j}^{-}\left(r_{t}\right), \tilde{\tau}_{j}\left(r_{t}\right)\right]\right) \geq 1-2 C_{+} e^{-\left(c_{-}\right) r_{t}}$. Then on $\left\{\mathcal{D}_{j} \subset\left[\tilde{\tau}_{j}^{-}\left(r_{t}\right), \tilde{\tau}_{j}\left(r_{t}\right)\right]\right\}$, for any $x \in \mathcal{D}_{j}$,

$$
\mathcal{L}_{j}\left(H_{j}\left(\tilde{L}_{j}\right), x\right) \leq A^{j}\left(\tilde{L}_{j}\right) \mathcal{L}_{B^{j}}\left[\tau^{B^{j}}(1), A^{j}(x) / A^{j}\left(\tilde{L}_{j}\right)\right] .
$$

Also with probability $\geq 1-2 C_{+} e^{-\left(c_{-}\right) r_{t}}, \mathcal{D}_{j} \subset\left[\tilde{\tau}_{j}^{-}\left(r_{t}\right), \tilde{\tau}_{j}\left(r_{t}\right)\right]$ so for any $x \in \mathcal{D}_{j}$,

$$
A^{j}\left(\tilde{\tau}_{j}^{-}\left(r_{t}\right)\right) \leq A^{j}(x) \leq A^{j}\left(\tilde{\tau}_{j}\left(r_{t}\right)\right)
$$

With Remark 2.3, Lemma 2.2, Fact 2.1 and (7.8), we obtain with a probability larger than $1-e^{-\left(c_{-}\right) r_{t}}$,

$$
\begin{aligned}
-e^{-h_{t} / 4} & \leq-e^{2 r_{t}} e^{-(1-1 / 2) h_{t}} \leq \frac{A^{j}\left(\tilde{\tau}_{j}^{-}\left(r_{t}\right)\right)}{A^{j}\left(\tilde{L}_{j}\right)} \leq \frac{A^{j}\left(\tilde{\tau}_{j}\left(r_{t}\right)\right)}{A^{j}\left(\tilde{L}_{j}\right)} \\
& \leq e^{2 r_{t}} e^{-(1-1 / 2) h_{t}} \leq e^{-h_{t} / 4}
\end{aligned}
$$

Therefore, applying (7.11) (with $\delta=e^{-h_{t} / 4}$ and $\varepsilon=\delta^{1 / 3}$ ), we obtain with a probability larger than $1-e^{-\left(c_{-}\right) r_{t}}$,

$$
\sup _{x \in \mathcal{D}_{j}} A^{j}\left(\tilde{L}_{j}\right) \mathcal{L}_{B^{j}}\left(\tau^{B^{j}}(1), A^{j}(x) / A^{j}\left(\tilde{L}_{j}\right)\right) \leq A^{j}\left(\tilde{L}_{j}\right) \mathcal{L}_{B^{j}}\left(\tau^{B^{j}}(1), 0\right)\left(1+e^{-h_{t} / 12}\right) .
$$

Collecting the different estimates we then obtain,

$$
\tilde{\nu}_{5}(y) \geq \mathbb{P}\left(\max _{1 \leq j \leq k-1} \mathcal{L}_{j}\left(H_{j}\left(\tilde{L}_{j}\right), \tilde{m}_{j}\right) \leq t \bar{\alpha}_{t}, \sum_{j=1}^{k-1} \frac{H_{j}\left(\tilde{L}_{j}\right)}{t} \leq y\right)-C_{+} e^{-\left(c_{-}\right) r_{t}}
$$

with $\bar{\alpha}_{t}:=\tilde{\alpha}_{t}\left(1+e^{-h_{t} / 12}\right)^{-1}$. We can then inverse the equality in law we have used above, and then obtain

$$
\tilde{\nu}_{5}(y) \geq F_{\bar{\alpha}_{t}}(y)-C_{+} e^{-\left(c_{-}\right) r_{t}},
$$

with $F_{\bar{\alpha}_{t}}$ defined in Lemma 5.2. Then we can follow the same lines as for the upper bound (especially computations after (5.9)), and obtain via Lemma 5.2 and by choosing $C_{0}$ large enough in such a way that $\left(c_{-}\right) r_{t} / \phi(t)=\left(c_{-}\right) C_{0}>\kappa(1+\delta)$ :

$$
\int_{\varepsilon}^{1-\varepsilon} \nu_{4}(y) \mathrm{d} \tilde{\nu}_{5}(y) \geq \int_{\varepsilon}^{1-\varepsilon} \nu_{4}(y) \mathrm{d} F_{\bar{\alpha}_{t}}^{+}(y)-o\left(n_{t}^{-1}\right) .
$$


Remark also that (5.22) implies the concentration of the local time at the $h_{t^{-}}$ minima: with probability larger than $1-C_{+} e^{-\left(c_{-}\right) r_{t}}$,

$$
\left|\sup _{y \in \mathcal{D}_{j}} \mathcal{L}_{j}\left(H_{j}\left(\tilde{L}_{j}\right), y\right)-\mathcal{L}_{j}\left(H_{j}\left(\tilde{L}_{j}\right), \tilde{m}_{j}\right)\right| \leq e^{-h_{t} / 12} \mathcal{L}_{j}\left(H_{j}\left(\tilde{L}_{j}\right), \tilde{m}_{j}\right) .
$$

We now work on $\nu_{4}(y)$. By the second part of Lemma 2.2 it is equal to

$$
\begin{aligned}
& E\left(\mathbb{P}_{\tilde{m}_{1}}^{W_{\kappa}}\left(\sup _{z \in \mathcal{D}_{1}} \mathcal{L}_{X^{\prime}}(t(1-y), z) \leq t \tilde{\alpha}_{t}, H^{\prime}\left(\tilde{L}_{1}\right)>t(1-y), H^{\prime}\left(\tilde{L}_{1}\right)<H^{\prime}\left(\tilde{L}_{1}^{-}\right)\right)\right) \\
= & : \tilde{\nu}_{4}(y),
\end{aligned}
$$

and by Lemma 5.3, $\tilde{\nu}_{4}(y) \geq f_{\tilde{\alpha}_{t}}^{-}(y)-o\left(n_{t}^{-1}\right)$. Therefore

$$
\int_{\varepsilon}^{1-\varepsilon} \nu_{4}(y) \mathrm{d} \tilde{\nu}_{5}(y) \geq \int_{\varepsilon}^{1-\varepsilon} f_{\tilde{\alpha}_{t}}^{-}(y) \mathrm{d} F_{\bar{\alpha}_{t}}^{+}(y)-o\left(n_{t}^{-1}\right) .
$$

From now on, the computations are very close from that of the upper bound (see (5.13) and below) and we do not give more details.

Proof of Lemmata 5.2, 5.3 and 5.4.

Proof of Lemma 5.2: This is a direct consequence of Proposition 3.5.

Proof of Lemma 5.3: To obtain the result we use a similar method than in Andreoletti and Diel (2011). That is to say, we study the inverse of the local time at $\tilde{m}_{1}$, and use our knowledge about $H\left(\tilde{L}_{1}\right)$. From the definitions of $f_{\gamma}$ and $\tilde{f}_{\gamma}$ we have easily $\tilde{f}_{\gamma}(x) \leq f_{\gamma}(x)$ for all $x$. So, to prove (5.1), we only need to prove the upper bound for $f_{\gamma}$ and the lower bound for $\tilde{f}_{\gamma}$. We fix $\varepsilon \in(0,1 / 2)$.

- Upper bound for $f_{\gamma}(x)$. Recall that $\sigma\left(u, \tilde{m}_{1}\right)=\inf \left\{s>0, \mathcal{L}\left(s, \tilde{m}_{1}\right) \geq u\right\}, u \geq 0$. First, notice that for $0<x<1, f_{\gamma}(x)$ is equal to

$$
\begin{aligned}
& E\left[\mathbb{P}_{\tilde{m}_{1}}^{W_{\kappa}}\left(\mathcal{L}\left(t(1-x), \tilde{m}_{1}\right) \leq \gamma t, H\left(\tilde{L}_{1}\right)>t(1-x), H\left(\tilde{L}_{1}\right)<H\left(\tilde{L}_{1}^{-}\right)\right)\right] \\
& =E\left[\mathbb{P}_{\tilde{m}_{1}}^{W_{\kappa}}\left(\sigma\left(\gamma t, \tilde{m}_{1}\right) \geq t(1-x), H\left(\tilde{L}_{1}\right)>t(1-x), H\left(\tilde{L}_{1}\right)<H\left(\tilde{L}_{1}^{-}\right)\right)\right] \\
& =E\left[\mathbb{P}_{\tilde{m}_{1}}^{W_{\kappa}}\left(H\left(\tilde{L}_{1}\right)>\sigma\left(\gamma t, \tilde{m}_{1}\right) \geq t(1-x), H\left(\tilde{L}_{1}\right)<H\left(\tilde{L}_{1}^{-}\right)\right)\right] \\
& \quad+E\left[\mathbb{P}_{\tilde{m}_{1}}^{W_{\kappa}}\left(\sigma\left(\gamma t, \tilde{m}_{1}\right)>H\left(\tilde{L}_{1}\right)>t(1-x), H\left(\tilde{L}_{1}\right)<H\left(\tilde{L}_{1}^{-}\right)\right)\right] .
\end{aligned}
$$

Let us first study the expectation in (5.25). On $\left\{H\left(\tilde{L}_{1}\right)>\sigma\left(\gamma t, \tilde{m}_{1}\right), H\left(\tilde{L}_{1}\right)<\right.$ $\left.H\left(\tilde{L}_{1}^{-}\right)\right\}$under $\mathbb{P}_{\tilde{m}_{1}}^{W_{\kappa}}, X$ remains between $\tilde{L}_{1}^{-}$and $\tilde{L}_{1}$ until time $\sigma\left(\gamma t, \tilde{m}_{1}\right)$ which is finite. On this event and under $\mathbb{P}_{\tilde{m}_{1}}^{W_{\kappa}}$, considering (1.12) and (1.13) as in Shi (1998, p. 248), the inverse of the local time can be written for $X$ starting at $\tilde{m}_{1}$ as

$$
\sigma\left(\gamma t, \tilde{m}_{1}\right)=\int_{\tilde{L}_{1}^{-}}^{\tilde{L}_{1}} e^{-\tilde{V}^{(1)}(z)} \mathcal{L}_{B}\left(\sigma_{B}(\gamma t, 0), A^{1}(z)\right) \mathrm{d} z=: I,
$$

where $A^{1}(z)=\int_{\tilde{m}_{1}}^{z} e^{\tilde{V}^{(1)}(y)} \mathrm{d} y$ and $B$ is a standard Brownian motion independent of $W_{\kappa}$, such that $B$ starts at $A^{1}\left(\tilde{m}_{1}\right)=0$ and is killed when it first hits $A^{1}\left(\tilde{L}_{1}\right)$. In (5.27), we integrate only between $\tilde{L}_{1}^{-}$and $\tilde{L}_{1}$ because under $\mathbb{P}_{\tilde{m}_{1}}^{W_{\kappa}}$, 
$e^{-\tilde{V}^{(1)}(z)} \mathcal{L}_{B}\left(\sigma_{B}(\gamma t, 0), A^{1}(z)\right)=\mathcal{L}\left(\sigma\left(\gamma t, \tilde{m}_{1}\right), z\right)=0$ for $z \notin\left[\tilde{L}_{1}^{-}, \tilde{L}_{1}\right]$ as explained after (5.26). We have

$$
I=\gamma t \int_{\tilde{L}_{1}^{-}}^{\tilde{L}_{1}} e^{-\tilde{V}^{(1)}(z)} \mathcal{L}_{\tilde{B}}\left(\sigma_{\tilde{B}}(1,0), \tilde{a}(z)\right) \mathrm{d} z
$$

with $\tilde{a}(z):=(\gamma t)^{-1} A^{1}(z)=(\gamma t)^{-1} \int_{\tilde{m}_{1}}^{z} e^{\tilde{V}^{(1)}(y)} \mathrm{d} y$ and where $\tilde{B}:=B\left((\gamma t)^{2}.\right) /(\gamma t)$. By scale invariance $\tilde{B}$ is also a standard Brownian motion that we still denote by $B$ in the sequel. Also, recall that $\sigma_{U}(r, y):=\inf \left\{s>0, \mathcal{L}_{U}(s, y)>r\right\}$ for $r>0$, $y \in \mathbb{R}$ is the inverse of the local time of the process $U$. Since we consider $X$ starting at $\tilde{m}_{1}$, we have $H\left(\tilde{L}_{1}\right)=H\left(\tilde{L}_{1}\right)-H\left(\tilde{m}_{1}\right)=U_{1}$, for which Proposition 3.5 gives

$$
\mathbb{E}\left(\mathbb{P}_{\tilde{m}_{1}}^{W_{\kappa}}\left\{\left|H\left(\tilde{L}_{1}\right)-\mathcal{H}_{1}\right| \leq \varepsilon_{t} \mathcal{H}_{1}\right\}\right)=\mathbb{P}\left(\left\{\left|U_{1} \mathcal{H}_{1}\right| \leq \varepsilon_{t} \mathcal{H}_{1}\right\}=: \mathcal{G}_{1}\right) \geq 1-e^{-D_{1} h_{t}},
$$

with $\varepsilon_{t}:=e^{-d_{1} h_{t}}$, if $\delta>0$ is chosen small enough. This will explain the appearance of $\mathcal{H}_{1}$ in $f_{\gamma}^{ \pm}(x)$. So, we now deal with $I$. Notice that $(\gamma t)^{-1} I$ can be split into two terms $(\gamma t)^{-1} I=I_{1}+I_{2}$, with

$$
I_{1}:=\int_{\tilde{\tau}_{1}^{-}\left(h_{t} / 2\right)}^{\tilde{\tau}_{1}\left(h_{t} / 2\right)} e^{-\tilde{V}^{(1)}(z)} \mathcal{L}_{B}\left(\sigma_{B}(1,0), \tilde{a}(z)\right) \mathrm{d} z,
$$

and $I_{2}:=(\gamma t)^{-1} I-I_{1} \geq 0$. We now prove that the main contribution in $(\gamma t)^{-1} I$ comes from $I_{1}$ and obtain its approximation in probability. Let $\varepsilon \in(0,1 / 100)$. First, using the second part of Lemma 2.2, followed by Remark 2.3, Fact 2.1 (ii) (for which we need $i \geq 2$ ), (7.8) and finally the first part of Lemma 2.2, we get

$$
\begin{aligned}
& P\left[\left|A^{1}\left(\tilde{\tau}_{1}^{-}\left(h_{t} / 2\right)\right)\right| \leq e^{h_{t}(1+\varepsilon) / 2},\left|A^{1}\left(\tilde{\tau}_{1}\left(h_{t} / 2\right)\right)\right| \leq e^{h_{t}(1+\varepsilon) / 2}\right] \\
= & P\left[\left|A^{2}\left(\tilde{\tau}_{2}^{-}\left(h_{t} / 2\right)\right)\right| \leq e^{h_{t}(1+\varepsilon) / 2},\left|A^{2}\left(\tilde{\tau}_{2}\left(h_{t} / 2\right)\right)\right| \leq e^{h_{t}(1+\varepsilon) / 2}\right] \\
\geq & 1-2 P\left[F^{+}\left(h_{t} / 2\right)>e^{h_{t}(1+\varepsilon) / 2}\right]-P\left[\overline{\mathcal{V}_{t}}\right] \geq 1-C_{+} e^{-\kappa \varepsilon h_{t} / 4} .
\end{aligned}
$$

Therefore, since $\tilde{a}\left(\tilde{\tau}_{1}^{-}\left(h_{t} / 2\right)\right) \leq \tilde{a}(z) \leq \tilde{a}\left(\tilde{\tau}_{1}\left(h_{t} / 2\right)\right)$ for all $z \in\left[\tilde{\tau}_{1}^{-}\left(h_{t} / 2\right), \tilde{\tau}_{1}\left(h_{t} / 2\right)\right]$,

$$
P\left(\forall z \in\left[\tilde{\tau}_{1}^{-}\left(h_{t} / 2\right), \tilde{\tau}_{1}\left(h_{t} / 2\right)\right],|\tilde{a}(z)| \leq e^{-(\log t)(1-3 \varepsilon) / 2}\right) \geq 1-C_{+} e^{-\kappa \varepsilon h_{t} / 4} .
$$

Also, using (7.15) and the second Ray-Knight theorem (see before (7.15)), we have

$$
\mathbb{P}\left(\sup _{|u| \leq e^{-(\log t)(1-3 \varepsilon) / 2}}\left|\mathcal{L}_{B}\left(\sigma_{B}(1,0), u\right)-1\right| \geq \widehat{\varepsilon}_{t}\right) \leq e^{-t^{\varepsilon} / 16} .
$$

with $\widehat{\varepsilon}_{t}:=t^{-(1-5 \varepsilon) / 4}$. So we obtain

$$
\mathbb{E}\left[\mathbb{P}_{\tilde{m}_{1}}^{W_{\kappa}}\left(\left|I_{1}-\tilde{R}_{1}\right| \leq \widehat{\varepsilon}_{t} \tilde{R}_{1}\right)\right] \geq 1-C_{+} e^{-\kappa \varepsilon h_{t} / 4},
$$

with $\tilde{R}_{1}:=\int_{\tilde{\tau}_{1}^{-}\left(h_{t} / 2\right)}^{\tilde{\tau}_{1}\left(h_{t} / 2\right)} e^{-\tilde{V}^{(1)}(z)} \mathrm{d} z$. We now prove that $I_{2}$ is negligible compared to the integral $\tilde{R}_{1}$ which appears in the previous equation, and then compared to $I_{1}$. First thanks to (7.16) and the second Ray-Knight theorem, we have

$$
\mathbb{E}\left[\mathbb{P}_{\tilde{m}_{1}}^{W_{\kappa}}\left(\sup _{z \in\left[\tilde{L}_{1}^{-}, \tilde{L}_{1}\right]} \mathcal{L}_{B}\left[\sigma_{B}(1,0), \tilde{a}(z)\right]>e^{\varepsilon \log t}\right)\right] \leq 2 e^{-\varepsilon \log t} .
$$


So with probability larger than $1-2 e^{-\varepsilon \log t}$, we have

$$
I_{2} \leq e^{\varepsilon \log t}\left(\int_{\tilde{L}_{1}^{-}}^{\tilde{\tau}_{1}^{-}\left(h_{t} / 2\right)} e^{-\tilde{V}^{(1)}(z)} \mathrm{d} z+\int_{\tilde{\tau}_{1}\left(h_{t} / 2\right)}^{\tilde{L}_{1}} e^{-\tilde{V}^{(1)}(z)} \mathrm{d} z\right)=: e^{\varepsilon \log t} I_{3} .
$$

By Lemma 6.8, with a probability larger than $1-2 e^{-\left(c_{-}\right) \varepsilon h_{t}}$ for large $t$,

$$
I_{3} \leq C_{+} h_{t}^{2} e^{-(1-\varepsilon) h_{t} / 2} .
$$

Also, by Lemma 3.6, with probability larger $1-e^{-\left(D_{-}\right) h_{t}}, \tilde{R}_{1}=R_{1}$ (which is the same $R_{1}$ as in (5.28)), which law is given by the sum of two independent copies of $F^{-}\left(h_{t} / 2\right)$. So using $(7.9)$, with a probability larger than $1-2 e^{-\left(D_{-}\right) h_{t}}$,

$$
\tilde{R}_{1}=R_{1} \geq e^{-\varepsilon h_{t} / 2} \text {. }
$$

We deduce from the last three inequalities that with a probability larger than $1-e^{-\left(c_{-}\right) \varepsilon h_{t}}$,

$$
I_{2}<R_{1} e^{-(1-5 \varepsilon) h_{t} / 2}=\tilde{R}_{1} e^{-(1-5 \varepsilon) h_{t} / 2} .
$$

Finally, using $(\gamma t)^{-1} I=I_{1}+I_{2}$ together with (5.32) and (5.33), we get

$$
\begin{gathered}
E\left[\mathbb { P } _ { \tilde { m } _ { 1 } } ^ { W _ { \kappa } } \left(\left|I-\gamma t R_{1}\right| \geq 2 t^{-(1-5 \varepsilon) / 4}(\gamma t) R_{1},\right.\right. \\
\left.\left.H\left(\tilde{L}_{1}\right)>\sigma\left(\gamma t, \tilde{m}_{1}\right), H\left(\tilde{L}_{1}\right)<H\left(\tilde{L}_{1}^{-}\right)\right)\right] \\
\leq \quad C_{+} e^{-\varepsilon\left(c_{-}\right) h_{t}} .
\end{gathered}
$$

We recall that by $(5.27), \sigma\left(\gamma t, \tilde{m}_{1}\right)=I$ on $\left\{H\left(\tilde{L}_{1}\right)>\sigma\left(\gamma t, \tilde{m}_{1}\right), H\left(\tilde{L}_{1}\right)<H\left(\tilde{L}_{1}^{-}\right)\right\}$ under $\mathbb{P}_{\tilde{m}_{1}}^{W_{\kappa}}$. Hence, combining (5.34) with (5.28) gives for large $t$ for every $x \in$ $[\varepsilon, 1-\varepsilon]$,

$$
\begin{aligned}
& \left\{H\left(\tilde{L}_{1}\right)>\sigma\left(\gamma t, \tilde{m}_{1}\right) \geq t(1-x), H\left(\tilde{L}_{1}\right)<H\left(\tilde{L}_{1}^{-}\right)\right\} \\
& \subset\left\{\frac{1}{R_{1}} \leq \frac{\gamma}{1-x}\left(1+\varepsilon_{t}^{\prime}\right), \mathcal{H}_{1}>t(1-x)\left(1-\varepsilon_{t}^{\prime}\right), H\left(\tilde{L}_{1}\right)>\sigma\left(\gamma t, \tilde{m}_{1}\right)\right\} \cup \mathcal{E}_{\varepsilon}^{1},
\end{aligned}
$$

where $\mathcal{E}_{\varepsilon}^{1}$ is such that $E\left[\mathbb{P}_{\tilde{m}_{1}}^{W_{\kappa}}\left(\mathcal{E}_{\varepsilon}^{1}\right)\right] \leq C_{+} e^{-\left(\varepsilon c_{-}\right) h_{t}}+e^{-D_{1} h_{t}}$ and where, as defined in the statement of the lemma, $\varepsilon_{t}^{\prime}=e^{-c_{2} h_{t}}$ with $c_{2}>0$ chosen small enough.

Now, let us study (5.26). On the event inside the probability in (5.26), $\sigma\left(\gamma t, \tilde{m}_{1}\right)$ might be infinite. We work under $\mathbb{P}_{\tilde{m}_{1}}^{W_{\kappa}}$. There exists a Brownian motion $B$ such that, with $T^{1}$ playing under $\mathbb{P}_{\tilde{m}_{1}}^{W_{\kappa}}$ the same role as $T$ does under $\mathbb{P}$ (see (1.12)), $H\left(\tilde{L}_{1}\right)=T^{1}\left(\tau^{B}\left(A^{1}\left(\tilde{L}_{1}\right)\right)\right)$ and $\sigma\left(\gamma t, \tilde{m}_{1}\right)=T^{1}\left(\sigma_{B}(\gamma t, 0)\right)$ (as in (5.27) and in Shi (1998) p. 248). Also by (1.12), notice for further use that under $\mathbb{P}_{\tilde{m}_{1}}^{W_{\kappa}}$,

$$
\mathcal{L}\left(\sigma\left(y t, \tilde{m}_{1}\right), z\right)=e^{-\tilde{V}^{(1)}(z)} \mathcal{L}_{B}\left(\sigma_{B}(y t, 0), A^{1}(z)\right), \quad z \in \mathbb{R}, y \in(0,+\infty) .
$$

So, we have

$$
\begin{aligned}
\sigma\left(\gamma t, \tilde{m}_{1}\right)>H\left(\tilde{L}_{1}\right) & \Leftrightarrow \sigma_{B}(\gamma t, 0)>\tau^{B}\left(A^{1}\left(\tilde{L}_{1}\right)\right) \\
& \Leftrightarrow \quad \mathcal{L}_{B}\left[\sigma_{B}(\gamma t, 0), 0\right]=\gamma t>\mathcal{L}_{B}\left[\tau^{B}\left(A^{1}\left(\tilde{L}_{1}\right)\right), 0\right] .
\end{aligned}
$$

Now, note that, as in (3.22) in the proof of Lemma 3.6, $\mathcal{L}_{B}\left[\tau^{B}\left(A^{1}\left(\tilde{L}_{1}\right)\right), 0\right]=$ $A^{1}\left(\tilde{L}_{1}\right) \mathcal{L}_{\tilde{B}}\left(\tau^{\tilde{B}}(1), 0\right)$, where $\tilde{B}:=B\left(\left(A^{1}\left(\tilde{L}_{1}\right)\right)^{2}.\right) / A^{1}\left(\tilde{L}_{1}\right)$. Also, by definition of $\mathbf{e}_{1}$ 
given in (3.22), we have $\mathcal{L}_{\tilde{B}}\left(\tau^{\tilde{B}}(1), 0\right)=\mathbf{e}_{1}$. As a consequence,

$$
\sigma\left(\gamma t, \tilde{m}_{1}\right)>H\left(\tilde{L}_{1}\right) \Leftrightarrow \gamma t>A^{1}\left(\tilde{L}_{1}\right) \mathbf{e}_{1} \Leftrightarrow \gamma t R_{1}>A^{1}\left(\tilde{L}_{1}\right) \mathbf{e}_{1} R_{1} .
$$

Then, according to $(3.18)$, we have $A^{1}\left(\tilde{L}_{1}\right) \geq\left(1-e^{-\left(d_{-}\right) h_{t}}\right) S_{1}$ with probability greater than $1-e^{-\left(D_{-}\right) h_{t}}$. Moreover, according to (5.28) and to the fact that under $\mathbb{P}_{\tilde{m}_{1}}^{W_{\kappa}}$ the diffusion $X$ starts at $\tilde{m}_{1}$, we have $\mathcal{H}_{1}=\mathbf{e}_{1} S_{1} R_{1} \geq\left(1+\varepsilon_{t}\right)^{-1} H\left(\tilde{L}_{1}\right)$ with probability greater than $1-e^{-\left(D_{-}\right) h_{t}}$. As a consequence,

$$
\sigma\left(\gamma t, \tilde{m}_{1}\right)>H\left(\tilde{L}_{1}\right) \Rightarrow \gamma t R_{1}>\left(1-e^{-\left(d_{-}\right) h_{t}}\right)\left(1+\varepsilon_{t}\right)^{-1} H\left(\tilde{L}_{1}\right),
$$

except on an event which probability $E\left[\mathbb{P}_{\tilde{m}_{1}}^{W_{\kappa}}().\right]$ is less than $2 e^{-\left(D_{-}\right) h_{t}}$. Combining this with (5.28) we get for large $t$ for every $x \in[\varepsilon, 1-\varepsilon]$,

$$
\begin{aligned}
& \left.\left\{\sigma\left(\gamma t, \tilde{m}_{1}\right)>H\left(\tilde{L}_{1}\right)>t(1-x), H\left(\tilde{L}_{1}\right)<H\left(\tilde{L}_{1}^{-}\right)\right)\right\} \\
& \subset\left\{\frac{1}{R_{1}} \leq \frac{\gamma}{1-x}\left(1+\varepsilon_{t}^{\prime}\right), \mathcal{H}_{1}>t(1-x)\left(1-\varepsilon_{t}^{\prime}\right), \sigma\left(\gamma t, \tilde{m}_{1}\right)>H\left(\tilde{L}_{1}\right)\right\} \cup \mathcal{E}_{\varepsilon}^{2},
\end{aligned}
$$

where $\mathcal{E}_{\varepsilon}^{2}$ is such that $E\left[\mathbb{P}_{\tilde{m}_{1}}^{W_{\kappa}}\left(\mathcal{E}_{\varepsilon}^{2}\right)\right] \leq 2 e^{-\left(D_{-}\right) h_{t}}+e^{-D_{1} h_{t}}$ and where, as before, $\varepsilon_{t}^{\prime}=e^{-c_{2} h_{t}}$ with $c_{2}>0$ possibly smaller than before.

Combining (5.25), (5.26) (5.35) and (5.38) with the strong Markov property, we get for large $t$ for every $x \in[\varepsilon, 1-\varepsilon]$, since $\phi(t)=o(\log t)$,

$$
\begin{aligned}
f_{\gamma}(x) & \leq \mathbb{P}\left(\frac{1}{R_{1}} \leq \frac{\gamma}{1-x}\left(1+\varepsilon_{t}^{\prime}\right), \mathcal{H}_{1}>t(1-x)\left(1-\varepsilon_{t}^{\prime}\right)\right)+o\left(n_{t}^{-1}\right) . \\
& =f_{\gamma}^{+}(x)+o\left(n_{t}^{-1}\right) .
\end{aligned}
$$

- Lower bound for $\tilde{f}_{\gamma}$. Let $\tilde{\gamma}:=\gamma\left(1+e^{-h_{t} / 12}\right)^{-1}$ and $y:=(1-x) /\left[R_{1}\left(1-4 \widehat{\varepsilon}_{t}\right)\right]$. We have to distinguish the cases $H\left(\tilde{L}_{1}\right)>\sigma\left(\tilde{\gamma} t, \tilde{m}_{1}\right)$ and $\sigma\left(\tilde{\gamma} t, \tilde{m}_{1}\right)>H\left(\tilde{L}_{1}\right)$. We work under $\mathbb{P}_{\tilde{m}_{1}}^{W_{\kappa}}$. On $\left\{y \leq \tilde{\gamma}, H\left(\tilde{L}_{1}\right)>\sigma\left(\tilde{\gamma} t, \tilde{m}_{1}\right) \geq t(1-x), H\left(\tilde{L}_{1}\right)<H\left(\tilde{L}_{1}^{-}\right)\right\}$, we can express the local time of $X$ at the inverse of its local time in $\tilde{m}_{1}$ at time $y t$ in terms of the standard Brownian motion driving the diffusion. More precisely by (5.36) and by scale invariance, there exists a Brownian motion $B$ such that for any $z \in \mathcal{D}_{1}$

$$
\mathcal{L}\left(\sigma\left(y t, \tilde{m}_{1}\right), z\right)=(y t) e^{-\tilde{V}^{(1)}(z)} \mathcal{L}_{B}\left(\sigma_{B}(1,0), \widehat{a}(z)\right)
$$

with $\widehat{a}(z):=(y t)^{-1} \int_{\tilde{m}_{1}}^{z} e^{\tilde{V}^{(1)}(u)} \mathrm{d} u=A^{1}(z) /(y t)$. Notice that by $(1.6), F^{-}\left(h_{t} / 2\right) \leq$ $\tau^{W_{\kappa}^{\uparrow}}\left(h_{t} / 2\right)$ in law, so $P\left[R_{1}>8 h_{t} / \kappa\right] \leq 2 P\left[F^{-}\left(h_{t} / 2\right)>4 h_{t} / \kappa\right] \leq 2 P\left[\tau^{W_{\kappa}^{\uparrow}}\left(h_{t} / 2\right)>\right.$ $\left.4 h_{t} / \kappa\right] \leq e^{-\left(c_{-}\right) h_{t}}$ for large $t$. Moreover, we prove with the same method used to prove (5.19) that $\tilde{\tau}^{-}\left(h_{t} / 2\right) \leq \tilde{m}_{1}-r_{t} \leq \tilde{m}_{1}+r_{t} \leq \tilde{\tau}\left(h_{t} / 2\right)$ with probability at least $1-C_{+} e^{-\left(c_{-}\right) h_{t}}$. This and (5.29) give $-e^{h_{t}(1+\varepsilon) / 2} \leq A^{1}\left[\tilde{\tau}^{-}\left(h_{t} / 2\right)\right] \leq A^{1}(z) \leq$ $A^{1}\left[\tilde{\tau}\left(h_{t} / 2\right)\right] \leq e^{h_{t}(1+\varepsilon) / 2}$ for any $z \in \mathcal{D}_{1}$ with probability $\geq 1-e^{-\left(c_{-}\right) \varepsilon h_{t}}$. So, for large $t$ for every $x \in[\varepsilon, 1-\varepsilon],|\widehat{a}(z)| \leq e^{h_{t}(1+\varepsilon) / 2} R_{1} /(t(1-x)) \leq e^{-(\log t)(1-3 \varepsilon) / 2}$ for these $z$ with such probability. Hence with the same method we used to prove (5.32) from (5.30) and (5.31), we get for large $t$ for every $x \in[\varepsilon, 1-\varepsilon]$,

$$
E\left(\mathbb{P}_{\tilde{m}_{1}}^{W_{\kappa}}\left(\sup _{z \in \mathcal{D}_{1}}\left|\mathcal{L}_{B}\left(\sigma_{B}(1,0), \widehat{a}(z)\right)-1\right| \leq \widehat{\varepsilon}_{t}\right)\right) \geq 1-2 e^{-\left(c_{-}\right) \varepsilon h_{t}}
$$


The above inequality together with (5.39) imply that for large $t$ for every $x \in$ $[\varepsilon, 1-\varepsilon]$,

$$
\begin{aligned}
& E\left(\mathbb { P } _ { \tilde { m } _ { 1 } } ^ { W _ { \kappa } } \left(\left\{\exists z \in \mathcal{D}_{1},\left|\mathcal{L}\left(\sigma\left(y t, \tilde{m}_{1}\right), z\right)-y t e^{-\tilde{V}^{(1)}(z)}\right| \geq 2 y t e^{-\tilde{V}^{(1)}(z)} \widehat{\varepsilon}_{t},\right.\right.\right. \\
& \left.\left.\left.y \leq \tilde{\gamma}, H\left(\tilde{L}_{1}\right)>\sigma\left(\tilde{\gamma} t, \tilde{m}_{1}\right) \geq t(1-x), H\left(\tilde{L}_{1}\right)<H\left(\tilde{L}_{1}^{-}\right)\right\}\right)\right) \\
\leq & 2 e^{-\left(c_{-}\right) \varepsilon h_{t}} .
\end{aligned}
$$

On $\left\{y \leq \tilde{\gamma}, H\left(\tilde{L}_{1}\right)>\sigma\left(\tilde{\gamma} t, \tilde{m}_{1}\right) \geq t(1-x), H\left(\tilde{L}_{1}\right)<H\left(\tilde{L}_{1}^{-}\right)\right\}$, if $t(1-x)>$ $\sigma\left(y t, \tilde{m}_{1}\right)$, then $\sigma\left(y t, \tilde{m}_{1}\right)-y t R_{1}<-4 t y R_{1} \widehat{\varepsilon}_{t}$, and by (5.34) (applied with $\gamma$ replaced by $y)$, this has on the previous event a probability $E\left(\mathbb{P}_{\tilde{m}_{1}}^{W_{\kappa}}().\right)$ less than $C_{+} e^{-\left(c_{-}\right) \varepsilon h_{t}}$. Thus on the previous event, we have $t(1-x) \leq \sigma\left(y t, \tilde{m}_{1}\right)$, except on a sub event of probability smaller than $C_{+} e^{-\left(c_{-}\right) \varepsilon h_{t}}$. This is true for every $x \in[\varepsilon, 1-\varepsilon]$ for large $t$.

Then since the local time is increasing in time, we have on the previous event for any $z \in \mathcal{D}_{1}, \mathcal{L}(t(1-x), z) \leq \mathcal{L}\left(\sigma\left(y t, \tilde{m}_{1}\right), z\right)$, which is according to (5.40) less than $y t e^{-V^{(1)}(z)}\left(1+2 \widehat{\varepsilon}_{t}\right) \leq y t\left(1+2 \widehat{\varepsilon}_{t}\right)$ for every $z \in \mathcal{D}_{1}$ with probability $E\left(\mathbb{P}_{\tilde{m}_{1}}^{W_{\kappa}}().\right)$ at least $1-2 e^{-\left(c_{-}\right) \varepsilon h_{t}}$. Combining this and the definition of our $y$ gives for large $t$, for every $x \in[\varepsilon, 1-\varepsilon]$,

$$
\begin{aligned}
& E\left(\mathbb { P } _ { \tilde { m } _ { 1 } } ^ { W _ { \kappa } } \left(\left\{\frac{\sup _{z \in \mathcal{D}_{1}} \mathcal{L}(t(1-x), z)}{t}>\frac{(1-x)}{R_{1}} \frac{1+2 \widehat{\varepsilon}_{t}}{1-4 \widehat{\varepsilon}_{t}}\right\}=: \overline{\mathcal{G}_{2}}\right.\right. \\
& \left.\left.\cap\left\{y \leq \tilde{\gamma}, H\left(\tilde{L}_{1}\right)>\sigma\left(\tilde{\gamma} t, \tilde{m}_{1}\right) \geq t(1-x), H\left(\tilde{L}_{1}\right)<H\left(\tilde{L}_{1}^{-}\right)\right\}\right)\right) \\
\leq & \left(2+C_{+}\right) e^{-(c-) \varepsilon h_{t}} .
\end{aligned}
$$

As a consequence, for $t$ large enough so that $1+2 \widehat{\varepsilon}_{t} \leq 1+e^{-h_{t} / 12}$, we have for every $x \in[\varepsilon, 1-\varepsilon]$,

$$
\begin{aligned}
& \left\{y \leq \tilde{\gamma}, H\left(\tilde{L}_{1}\right)>\sigma\left(\tilde{\gamma} t, \tilde{m}_{1}\right) \geq t(1-x), H\left(\tilde{L}_{1}\right)<H\left(\tilde{L}_{1}^{-}\right)\right\} \\
\subset & \left\{\sup _{z \in \mathcal{D}_{1}} \mathcal{L}(t(1-x), z) \leq y\left(1+2 \widehat{\varepsilon}_{t}\right) t \leq \gamma t, H\left(\tilde{L}_{1}\right)>\sigma\left(\tilde{\gamma} t, \tilde{m}_{1}\right)\right\} \cup \mathcal{E}_{\varepsilon}^{3}
\end{aligned}
$$

by definition of $\tilde{\gamma}$, where $\mathcal{E}_{\varepsilon}^{3}$ is such that $E\left(\mathbb{P}_{\tilde{m}_{1}}^{W_{\kappa}}\left(\mathcal{E}_{\varepsilon}^{3}\right)\right) \leq\left(2+C_{+}\right) e^{-\left(c_{-}\right) \varepsilon h_{t}}$.

On the other hand, from the definition of $\sigma\left(., \tilde{m}_{1}\right),(5.23)$ and the definition of $\tilde{\gamma}$, we have for large $t$ for every $x \in[\varepsilon, 1-\varepsilon]$,

$$
\begin{aligned}
& \left\{y \leq \tilde{\gamma}, \sigma\left(\tilde{\gamma} t, \tilde{m}_{1}\right)>H\left(\tilde{L}_{1}\right)>t(1-x), H\left(\tilde{L}_{1}\right)<H\left(\tilde{L}_{1}^{-}\right)\right\} \\
\subset & \left\{\mathcal{L}\left(H\left(\tilde{L}_{1}\right), \tilde{m}_{1}\right) \leq \tilde{\gamma} t, \sigma\left(\tilde{\gamma} t, \tilde{m}_{1}\right)>H\left(\tilde{L}_{1}\right)>t(1-x), H\left(\tilde{L}_{1}\right)<H\left(\tilde{L}_{1}^{-}\right)\right\} \\
\subset & \left\{\sup _{z \in \mathcal{D}_{1}} \mathcal{L}\left(H\left(\tilde{L}_{1}\right), z\right) \leq \gamma t, \sigma\left(\tilde{\gamma} t, \tilde{m}_{1}\right)>H\left(\tilde{L}_{1}\right)>t(1-x)\right\} \cup \mathcal{E}_{\varepsilon}^{4} \\
\subset & \left\{\sup _{z \in \mathcal{D}_{1}} \mathcal{L}(t(1-x), z) \leq \gamma t, \sigma\left(\tilde{\gamma} t, \tilde{m}_{1}\right)>H\left(\tilde{L}_{1}\right)\right\} \cup \mathcal{E}_{\varepsilon}^{4},
\end{aligned}
$$

where $\mathcal{E}_{\varepsilon}^{4}$ is the event where $(5.23)$ fails, it is such that $E\left(\mathbb{P}_{\tilde{m}_{1}}^{W_{\kappa}}\left(\mathcal{E}_{\varepsilon}^{4}\right)\right) \leq C_{+} e^{-\left(c_{-}\right) r_{t}}$. 
Combining (5.42) and (5.43) we get for large $t$ for every $x \in[\varepsilon, 1-\varepsilon]$, under $\mathbb{P}_{\tilde{m}_{1}}^{W_{\kappa}}$,

$$
\left\{y \leq \tilde{\gamma}, H\left(\tilde{L}_{1}\right)>t(1-x), H\left(\tilde{L}_{1}\right)<H\left(\tilde{L}_{1}^{-}\right)\right\} \subset\left\{\sup _{z \in \mathcal{D}_{1}} \mathcal{L}(t(1-x), z) \leq \gamma t\right\} \cup \mathcal{E}_{\varepsilon}^{5},
$$

where $\mathcal{E}_{\varepsilon}^{5}$ is such that $E\left(\mathbb{P}_{\tilde{m}_{1}}^{W_{\kappa}}\left(\mathcal{E}_{\varepsilon}^{5}\right)\right) \leq C_{+} e^{-\left(c_{-}\right) r_{t}}=C_{+} e^{-\left(c_{-}\right) C_{0} \phi(t)}=o\left(n_{t}^{-1}\right)$ as $t \rightarrow+\infty$ is we choose $C_{0}$ large enough. Combining this with (5.28), (3.2) and Proposition 3.5, we obtain for large $t$ for every $x \in[\varepsilon, 1-\varepsilon]$,

$$
\begin{aligned}
\tilde{f}_{\gamma}(x) & \geq \mathbb{P}\left(\frac{(1-x)}{R_{1}} \leq \gamma\left(1-\varepsilon_{t}^{\prime}\right), \mathbf{e}_{1} S_{1} R_{1}>t(1-x)\left(1+\varepsilon_{t}^{\prime}\right)\right)-o\left(n_{t}^{-1}\right) \\
& =f_{\gamma}^{-}(x)-o\left(n_{t}^{-1}\right),
\end{aligned}
$$

where the constant $c_{2}$ in the definition of $\varepsilon_{t}^{\prime}=e^{-c_{2} h_{t}}$ has been decreased if necessary. This proves the lower bound for $\tilde{f}_{\gamma}(x)$ and then finishes the proof of the lemma.

Proof of Lemma 5.4: Let $0<a<1 / 4$. We start with (5.2). By Proposition 3.5, the $\mathcal{H}_{i}, i \geq 1$ are i.i.d., so $\overline{\mathcal{H}}_{k-1}$ and $\overline{\mathcal{H}}_{k}-\overline{\mathcal{H}}_{k-1}=\mathcal{H}_{k}$ are independent for $k \geq 1$. Thus for $t>0$,

$$
\begin{aligned}
& \sum_{1 \leq k \leq n_{t}} \mathbb{P}\left[\overline{\mathcal{H}}_{k}>1-a / 2,1-2 a<\overline{\mathcal{H}}_{k-1} \leq 1-3 a / 4\right] \\
= & \int_{1-2 a}^{1-3 a / 4} d \mu_{t}(x) e^{\kappa \phi(t)} \mathbb{P}\left[\mathcal{H}_{1}>1-x-a / 2\right],
\end{aligned}
$$

where the measure $\mu_{t}$ is defined by $\int_{0}^{x} d \mu_{t}(y):=e^{-\kappa \phi(t)} \sum_{1 \leq k \leq n_{t}} \mathbb{P}\left[\overline{\mathcal{H}}_{k-1} \leq x\right]$. We know that $\mu_{t}$ converges vaguely as $t \rightarrow+\infty$ to the measure $\mu$ which has a density with respect to the Lebesgue measure equal to $\left(\Gamma(\kappa) \mathcal{C}_{\kappa}\right)^{-1} x^{\kappa-1} \mathbb{1}_{x>0}$, with $\mathcal{C}_{\kappa}>0$ (see Lemma 6.2). Also thanks to Lemma 4.1, $e^{\kappa \phi(t)} P\left[\mathcal{H}_{1} / t>x\right]$ converges uniformly on every compact subset of $(0,+\infty)$ to $\mathcal{C}_{\kappa} x^{-\kappa} / \Gamma(1-\kappa)$. Therefore,

$$
\begin{aligned}
& \lim _{t \rightarrow+\infty} \sum_{1 \leq k \leq n_{t}} \mathbb{P}\left[\overline{\mathcal{H}}_{k}>1-a / 2,1-2 a<\overline{\mathcal{H}}_{k-1} \leq 1-3 a / 4\right] \\
& =\frac{1}{\Gamma(\kappa) \Gamma(1-\kappa)} \int_{1-2 a}^{1-3 a / 4} x^{\kappa-1}(1-x-a / 2)^{-\kappa} \mathrm{d} x \\
& \leq \mathrm{const} \times a^{1-\kappa} .
\end{aligned}
$$

For (5.3), we apply (6.2) with $r=\varepsilon \in(0,1 / 2)$ and $s=1-\varepsilon$, which gives

$$
\begin{aligned}
& \lim _{t \rightarrow+\infty} \mathbb{P}\left(\varepsilon t \leq H\left(m_{N_{t}}\right) \leq(1-\varepsilon) t\right) \\
& =1-\frac{\sin (\pi \kappa)}{\pi}\left(\int_{0}^{\varepsilon} x^{\kappa-1}(1-x)^{-\kappa} \mathrm{d} x+\int_{1-\varepsilon}^{1} x^{\kappa-1}(1-x)^{-\kappa} \mathrm{d} x\right) \\
& \geq 1-\frac{\sin (\pi \kappa)}{\pi}\left(\frac{(1-\varepsilon)^{-\kappa}}{\kappa} \varepsilon^{\kappa}+\frac{(1-\varepsilon)^{\kappa-1}}{1-\kappa} \varepsilon^{1-\kappa}\right),
\end{aligned}
$$

which implies the result.

Proof of Theorem 1.3: The proof of this theorem is a direct consequence of Propositions 5.1 and 1.4 and of Lemmata 4.4 and 4.5. Notice that the proof of the upper bound does not use the proof of the lower bound, but we use the upper bound 
for the proof of the lower bound. In particular from the upper bound of Theorem 1.3 (which makes use of the upper bound of Proposition 5.1 but not of its lower bound), we have $\lim \sup _{t \rightarrow+\infty} \mathbb{P}\left(\mathcal{L}^{*}(t)<2 \tilde{w}_{t}\right) \leq \mathbb{P}\left(\mathcal{Y}_{1}^{\natural}\left(\mathcal{Y}_{2}^{-1}(1)^{-}\right) \leq \varepsilon\right)$ for any $\varepsilon>0$ as $\lim _{t \rightarrow+\infty} \tilde{w}_{t} / t=0$. From this, as $\mathcal{Y}_{1}^{\natural}\left(\mathcal{Y}_{2}^{-1}(1)^{-}\right)$is positive, we obtain $\lim _{t \rightarrow+\infty} \mathbb{P}\left(\mathcal{L}^{*}(t)<2 \tilde{w}_{t}\right)=0$, which proves assertion (5.18) at the beginning of the proof of the lower bound of Proposition 5.1.

Thanks to Proposition 5.1 and to the remark before this proposition, we only need to study the convergence of $\mathcal{P}_{1}^{ \pm}$(the limit when $t$ goes to infinity and then the limit when $\varepsilon$ goes to 0$)$. The latter can be written in term of functionals of $\left(Y_{1}, Y_{2}\right)^{t}$ as follows. Let $\mathbb{Y}_{t}:=\left(Y_{2}^{t}\right)^{-1}(1-2 \varepsilon)$; we have $\mathcal{N}_{t}^{2 \varepsilon} e^{-\kappa \phi(t)}=\mathbb{Y}_{t}$, and

$$
\begin{array}{r}
\mathcal{P}_{1}^{ \pm}=P\left[\left(1-Y_{2}^{t}\left(\mathbb{Y}_{t}^{-}\right)\right) \frac{Y_{1}^{t}\left(\mathbb{Y}_{t}\right)-Y_{1}^{t}\left(\mathbb{Y}_{t}^{-}\right)}{Y_{2}^{t}\left(\mathbb{Y}_{t}\right)-Y_{2}^{t}\left(\mathbb{Y}_{t}^{-}\right)} \leq \alpha_{t}^{ \pm},\left(Y_{1}^{t}\right)^{\natural}\left(\mathbb{Y}_{t}^{-}\right) \leq \alpha_{t}^{ \pm}\right] \\
=P\left[\left(1-\tilde{K}_{I, 1-2 \varepsilon}^{-}\left(\left(Y_{1}, Y_{2}\right)^{t}\right)\right) \frac{K_{I, 1-2 \varepsilon}\left(\left(Y_{1}, Y_{2}\right)^{t}\right)-K_{I, 1-2 \varepsilon}^{-}\left(\left(Y_{1}, Y_{2}\right)^{t}\right)}{\tilde{K}_{I, 1-2 \varepsilon}\left(\left(Y_{1}, Y_{2}\right)^{t}\right)-\tilde{K}_{I, 1-2 \varepsilon}^{-}\left(\left(Y_{1}, Y_{2}\right)^{t}\right)} \leq \alpha_{t}^{ \pm},\right. \\
\left.J_{I, 1-2 \varepsilon}^{-}\left(\left(Y_{1}, Y_{2}\right)^{t}\right) \leq \alpha_{t}^{ \pm}\right],
\end{array}
$$

with the notation $K_{I, a}, \tilde{K}_{I, a}, \ldots$ introduced in (4.25) and before. The hypotheses of Lemma 4.5 are: finite number of large jumps on compact intervals, strictly increasing, starting at 0 , and jumping over 1 without reaching it. These properties are naturally almost surely satisfied by a $\kappa$-stable subordinator so, almost surely, the paths of $\left(\mathcal{Y}_{1}, \mathcal{Y}_{2}\right)$ satisfy the hypotheses of Lemma 4.5 (see e.g. Bertoin, 1996 III.2 p. 75). Therefore they are points of continuity for $J_{I, 1-2 \varepsilon}^{-}, K_{I, 1-2 \varepsilon}^{-}, K_{I, 1-2 \varepsilon}$, $\tilde{K}_{I, 1-2 \varepsilon}^{-}$and $\tilde{K}_{I, 1-2 \varepsilon}$. Combining this continuity with Proposition 1.4, continuous mapping theorem, and replacing the functionals by their expressions, we obtain, when $t$ goes to infinity, the convergence of $\mathcal{P}_{1}^{ \pm}$to

$$
\begin{array}{r}
P\left[\left(1-\mathcal{Y}_{2}\left(\mathcal{Y}_{2}^{-1}(1-2 \varepsilon)^{-}\right)\right) \frac{\mathcal{Y}_{1}\left(\mathcal{Y}_{2}^{-1}(1-2 \varepsilon)\right)-\mathcal{Y}_{1}\left(\mathcal{Y}_{2}^{-1}(1-2 \varepsilon)^{-}\right)}{\mathcal{Y}_{2}\left(\mathcal{Y}_{2}^{-1}(1-2 \varepsilon)\right)-\mathcal{Y}_{2}\left(\mathcal{Y}_{2}^{-1}(1-2 \varepsilon)^{-}\right)} \leq \alpha,\right. \\
\left.\mathcal{Y}_{1}^{\natural}\left(\mathcal{Y}_{2}^{-1}(1-2 \varepsilon)^{-}\right) \leq \alpha\right] .
\end{array}
$$

Then, note that almost surely $\mathcal{Y}_{2}\left(\mathcal{Y}_{2}^{-1}(1)^{-}\right)<1$ so we have a.s. $\mathcal{Y}_{2}^{-1}(1-2 \varepsilon)=$ $\mathcal{Y}_{2}^{-1}(1)$ for all $\varepsilon$ small enough. We deduce that the above expression converges to the repartition function of $\max \left(\mathcal{I}_{1}, \mathcal{I}_{2}\right)$ (see (1.5) for definitions of $\mathcal{I}_{1}$ and $\mathcal{I}_{2}$ ) when $\varepsilon$ goes to 0 , and this yields Theorem 1.3.

\subsection{Favorite site (proof of Theorem 1.5).}

Thanks to Section 3, we know precisely the nature of the contribution of each $h_{t^{-}}$ valley to the local time. The difficulty in proving Theorem 1.3 was the need to consider only a part of the contribution of the last $h_{t}$-valley. The proofs of the first two points (1.8) and (1.9) of Theorem 1.5 are thus easier to obtain, since they do not require to "cut" the contribution of any valley. Let us prove the first point (1.8) 
(the second one, (1.9), is obtained similarly). We have, using (2.7),

$$
\begin{aligned}
& \mathbb{P}\left[\mathcal{L}^{*}\left(H\left(m_{N_{t}+1}\right)\right) \leq \alpha t\right] \\
& \leq \mathbb{P}\left(\mathcal{L}^{*}\left(H\left(\tilde{L}_{N_{t}}\right)\right) \leq \alpha t, \mathcal{Q}, \mathcal{V}_{t}\right)+\mathbb{P}(\overline{\mathcal{Q}})+P\left(\overline{\mathcal{V}}_{t}\right)+\mathbb{P}\left(\overline{\mathcal{B}_{3}\left(n_{t}\right)}\right) \\
& \leq \mathbb{P}\left(\sup _{1 \leq j \leq N_{t}} \ell_{j} / t \leq\left(1-\varepsilon_{t}\right)^{-1} \alpha, \mathcal{Q}, \mathcal{V}_{t}\right)+\mathbb{P}(\overline{\mathcal{Q}})+o(1),
\end{aligned}
$$

where we fixed some $\varepsilon>0$ and $\mathcal{Q}:=\left\{\varepsilon t \leq H\left(m_{N_{t}}\right) \leq(1-\varepsilon) t, 1 \leq N_{t} \leq n_{t}\right\}$ as after (5.4) (from there we see that $\lim _{\varepsilon \rightarrow 0} \lim _{t \rightarrow+\infty} \mathbb{P}(\overline{\mathcal{Q}})=0$ ). In the last inequality we used Proposition 3.5, Lemma 2.2 and Lemma 3.2. To lighten notation, let $\tilde{\alpha}_{t}:=\left(1-\varepsilon_{t}\right)^{-1} \alpha$. We have

$$
\begin{aligned}
& \mathbb{P}\left(\sup _{1 \leq j \leq N_{t}} \ell_{j} / t \leq \tilde{\alpha}_{t}, \mathcal{Q}, \mathcal{V}_{t}\right) \\
\leq & \mathbb{P}\left(\sup _{1 \leq j \leq N_{t}} \ell_{j} / t \leq \tilde{\alpha}_{t}, \mathcal{B}_{1}\left(n_{t}\right), \mathcal{Q}, \mathcal{V}_{t}\right)+\mathbb{P}\left(\overline{\mathcal{B}_{1}\left(n_{t}\right)}\right) \\
\leq & \mathbb{P}\left(\sup _{1 \leq j \leq N_{t}} \ell_{j} / t \leq \tilde{\alpha}_{t}, \overline{\mathcal{H}}_{N_{t}} \geq 1-\delta_{t}^{\prime}, \overline{\mathcal{H}}_{N_{t}-1} \leq 1-\varepsilon+\delta_{t}^{\prime}, \mathcal{Q}\right)+o(1),
\end{aligned}
$$

with $\delta_{t}^{\prime}=3 \tilde{v}_{t} / t$ and where we used (3.1) together with Proposition 3.5. Partitioning on the values of $N_{t}$ we get that the above is less than

$$
\sum_{1 \leq k \leq n_{t}} \mathbb{P}\left(\sup _{1 \leq j \leq k} \ell_{j} / t \leq \tilde{\alpha}_{t}, \overline{\mathcal{H}}_{k} \geq 1-\delta_{t}^{\prime}, \overline{\mathcal{H}}_{k-1} \leq 1-\varepsilon+\delta_{t}^{\prime}, \mathcal{Q}\right)+o(1) .
$$

Since the sum $\sum_{1}$ defined in the proof of the upper bound of Proposition 5.1 (see (5.15) and below) is smaller than $s(\varepsilon, t)$ satisfying $\lim _{\varepsilon \rightarrow 0} \lim _{t \rightarrow+\infty} s(\varepsilon, t)=0$, we can intersect the event on the above probability with $\left\{k=\mathcal{N}_{t}^{2 \varepsilon}\right\}$ and get

$$
\mathbb{P}\left[\mathcal{L}^{*}\left(H\left(m_{N_{t}+1}\right)\right) \leq \alpha t\right] \leq \mathbb{P}\left(\sup _{1 \leq j \leq \mathcal{N}_{t}^{2 \varepsilon}} \ell_{j} / t \leq \tilde{\alpha}_{t}\right)+\mathbb{P}(\overline{\mathcal{Q}})+s(\varepsilon, t)+o(1) .
$$

Then, as in the proof of Theorem 1.3 we have that $\left(\mathcal{Y}_{1}, \mathcal{Y}_{2}\right)$ almost surely satisfies the hypothesis of Lemma 4.5, and is therefore almost surely a point of continuity for $J_{I, 12-\varepsilon}$ defined just above (4.25). From this continuity, Proposition 1.4 and the continuous mapping theorem we get

$$
\sup _{1 \leq j \leq \mathcal{N}_{t}^{2 \varepsilon}} \ell_{j} / t=J_{I, 1-2 \varepsilon}\left(\left(Y_{1}, Y_{2}\right)^{t}\right) \underset{t \rightarrow+\infty}{\stackrel{\text { 亗 }}{\rightarrow}} J_{I, 1-2 \varepsilon}\left(\mathcal{Y}_{1}, \mathcal{Y}_{2}\right)=\mathcal{Y}_{1}^{\natural}\left(\mathcal{Y}_{2}^{-1}(1-2 \varepsilon)\right) .
$$

Then, as in the proof of Theorem 1.3 we have almost surely $\mathcal{Y}_{2}^{-1}(1-2 \varepsilon)=\mathcal{Y}_{2}^{-1}(1)$ for all $\varepsilon$ small enough so $\mathcal{Y}_{1}^{\natural}\left(\mathcal{Y}_{2}^{-1}(1-2 \varepsilon)\right)$ converges almost surely to $\mathcal{Y}_{1}^{\natural}\left(\mathcal{Y}_{2}^{-1}(1)\right)$ when $\varepsilon$ goes to 0 . Thus, we get

$$
\limsup _{t \rightarrow+\infty} \mathbb{P}\left[\mathcal{L}^{*}\left(H\left(m_{N_{t}+1}\right)\right) \leq \alpha t\right] \leq \mathbb{P}\left(\mathcal{Y}_{1}^{\natural}\left(\mathcal{Y}_{2}^{-1}(1)\right) \leq \alpha\right)
$$

A lower bound is proved similarly, so we get the following, proving (1.8):

$$
\lim _{t \rightarrow+\infty} \mathbb{P}\left[\mathcal{L}^{*}\left(H\left(m_{N_{t}+1}\right)\right) \leq \alpha t\right]=\mathbb{P}\left(\mathcal{Y}_{1}^{\natural}\left(\mathcal{Y}_{2}^{-1}(1)\right) \leq \alpha\right)
$$


To obtain the result (1.10) for the favorite site, we first argue that we essentially need to obtain the asymptotic behavior of $N_{t}^{*} / N_{t}$, where $N_{t}^{*}:=\min \{j \geq$ $\left.1, \mathcal{L}\left(m_{j}, t\right)=\max _{1 \leq k \leq N_{t}} \mathcal{L}\left(m_{k}, t\right)\right\}$. Indeed, define for any $\varepsilon \in(0,1 / 2)$,

$$
\begin{aligned}
& \mathcal{K}_{1}:=\left\{(1-\varepsilon) m_{N_{t}} \leq X(t) \leq(1+\varepsilon) m_{N_{t}}\right\}, \\
& \mathcal{K}_{2}:=\left\{(1-\varepsilon) m_{N_{t}^{*}} \leq F_{t}^{*} \leq(1+\varepsilon) m_{N_{t}^{*}}\right\} .
\end{aligned}
$$

Then, we have, $\lim _{t \rightarrow+\infty} \mathbb{P}\left(\mathcal{K}_{1}\right)=1$ by the localization result Theorem 1.2 combined with the fact that $X(t) / t^{\kappa}$ converges in law under $\mathbb{P}$ to a positive limit as $t \rightarrow+\infty$ by Kawazu and Tanaka (1997).

Let us now justify that $\lim _{t \rightarrow+\infty} \mathbb{P}\left(\mathcal{K}_{2}\right)=1$. According to $(5.18)$ proved at the start of the proof of Theorem 1.3, to Lemma 3.4 and (3.3), we have

$$
\mathbb{P}\left(\sup _{x \in \mathbb{R}} \mathcal{L}(t, x) \geq 2 \tilde{w}_{t}, \mathcal{B}_{4}\left(n_{t}\right), N_{t} \leq n_{t}\right) \underset{t \rightarrow+\infty}{\longrightarrow} 1
$$

Notice that on the event inside the above probability, for $t$ large enough so that $2 \tilde{w}_{t} \geq t e^{-2 \phi(t)}$, we have $F_{t}^{*} \in \mathcal{D}_{N_{t}^{*}}\left(\right.$ recall the definition of $\mathcal{D}_{j}$ in (3.13)). Since $\mathcal{D}_{N_{t}^{*}}$ is centered at $m_{N_{t}^{*}}$ and its half-length is deterministic and equal to $r_{t}=C_{0} \phi(t)$ we only need to justify that

$$
\mathbb{P}\left(\varepsilon m_{N_{t}^{*}} \geq C_{0} \phi(t)\right) \underset{t \rightarrow+\infty}{\longrightarrow} 1 .
$$

We have $m_{N_{t}^{*}} \geq m_{1}$ and $\mathbb{P}\left(m_{1} \geq C_{0} \phi(t) / \varepsilon\right) \geq \mathbb{P}\left(\tilde{m}_{1} \geq C_{0} \phi(t) / \varepsilon\right)-o(1)$ by Lemma 2.2. So using (6.13), we thus deduce that $\lim _{t \rightarrow+\infty} \mathbb{P}\left(\mathcal{K}_{2}\right)=1$.

We can now write for $x>0$,

$$
\mathbb{P}\left[\frac{F_{t}^{*}}{X(t)} \leq x\right]=\mathbb{P}\left[\frac{F_{t}^{*}}{X(t)} \leq x, \mathcal{K}_{1}, \mathcal{K}_{2}\right]+v(\varepsilon, t) \leq \mathbb{P}\left[\frac{m_{N_{t}^{*}}}{m_{N_{t}}} \leq x \frac{1+\varepsilon}{1-\varepsilon}\right]+v(\varepsilon, t) .
$$

where $v(\varepsilon, t) \geq 0$, satisfies $\lim _{\varepsilon \rightarrow 0} \lim _{t \rightarrow+\infty} v(\varepsilon, t)=0$. Similarly, we have

$$
\mathbb{P}\left[\frac{F_{t}^{*}}{X(t)} \leq x\right] \geq \mathbb{P}\left[\frac{m_{N_{t}^{*}}}{m_{N_{t}}} \leq x \frac{1-\varepsilon}{1+\varepsilon}\right]-v(\varepsilon, t) .
$$

Hence, we obtain

$$
\mathbb{P}\left[\frac{m_{N_{t}^{*}}}{m_{N_{t}}} \leq x \frac{1-\varepsilon}{1+\varepsilon}\right]-v(\varepsilon, t) \leq \mathbb{P}\left[\frac{F_{t}^{*}}{X(t)} \leq x\right] \leq \mathbb{P}\left[\frac{m_{N_{t}^{*}}}{m_{N_{t}}} \leq x \frac{1+\varepsilon}{1-\varepsilon}\right]+v(\varepsilon, t) .
$$

So, we observe that we only have to study the random variable $\frac{m_{N_{t}^{*}}}{m_{N_{t}}}$. For that we first remark that $N_{t}^{*}$ and $N_{t}$ diverge when $t$ goes to infinity. Indeed by Lemma 6.1 , the correct normalisation for the convergence in law of $N_{t}$ is $e^{\kappa \phi(t)}$, so $\mathbb{P}\left(N_{t} \geq\right.$ $\left.e^{(1-\varepsilon) \kappa \phi(t)}\right)=1-o(1)$. For $N_{t}^{*}$, we first notice that the previous result for $N_{t}$ also gives for $t$ large, $\mathbb{P}\left(N_{t} \geq e^{(1-\varepsilon / 2) \kappa \phi(t)}\right)=1-o(1)$. Therefore

$$
\mathbb{P}\left(N_{t}^{*} \leq e^{(1-\varepsilon) \kappa \phi(t)}\right) \leq \mathbb{P}\left(\max _{k \leq e^{(1-\varepsilon) \kappa \phi(t)}} \mathcal{L}\left(m_{k}, t\right) \geq \max _{k<e^{(1-\varepsilon / 2) \kappa \phi(t)}} \mathcal{L}\left(m_{k}, t\right)\right)+o(1) .
$$

Now, since $\mathcal{L}\left(m_{k}, t\right)=\mathcal{L}\left(\tilde{m}_{k}, H\left(\tilde{L}_{k}\right) \wedge\left(H\left(\tilde{m}_{k}\right)+H_{\tilde{m}_{k} \rightarrow \tilde{L}_{k}^{-}}\right)\right)=: \widehat{\ell}_{k}$ for $k<N_{t}$ on $\mathcal{V}_{t} \cap\left\{N_{t} \leq n_{t}\right\} \cap \mathcal{B}_{2}\left(n_{t}\right)$ which has probability $1-o(1)$ by Lemmas 2.2 and 3.1,

$$
\begin{aligned}
& \mathbb{P}\left(\max _{k \leq e^{(1-\varepsilon) \kappa \phi(t)}} \mathcal{L}\left(m_{k}, t\right) \geq \max _{k<e^{(1-\varepsilon / 2) \kappa \phi(t)}} \mathcal{L}\left(m_{k}, t\right)\right) \\
\leq & \mathbb{P}\left(\max _{k \leq e^{(1-\varepsilon) \kappa \phi(t)}} \widehat{\ell}_{k} \geq \max _{k<e^{(1-\varepsilon / 2) \kappa \phi(t)}} \widehat{\ell}_{k}\right)+o(1),
\end{aligned}
$$


with $\left(\widehat{\ell}_{k}, k \leq e^{(1-\varepsilon / 2) \kappa \phi(t)}\right)$ i.i.d. random variables under $\mathbb{P}$ by strong Markov property and the second part of Lemma 2.2, and with queue distributions given by (4.1) and Proposition 3.5.

It is then clear that for large $t, \mathbb{P}\left(\max _{k \leq e^{(1-\varepsilon) \kappa \phi(t)}} \widehat{\ell}_{k} \geq \max _{k<e^{(1-\varepsilon / 2) \kappa \phi(t)}} \widehat{\ell}_{k}\right)=o(1)$, and we therefore obtain that $\mathbb{P}\left(N_{t}^{*} \geq e^{(1-\varepsilon) \kappa \phi(t)}\right)=1-o(1)$.

Then, following the work of Faggionato (2009), we know that $\left(m_{i}-m_{i-1}, i \geq 2\right)$ are i.i.d. random variables with a known Laplace transform (given by (2.19) in Faggionato, 2009), this allows to compute the first and fourth moments of $\Delta m_{1}:=$ $m_{2}-m_{1}$ and therefore obtain after an elementary but tedious computation that for large $t, \mathbb{E}\left(\Delta m_{1}\right) \sim C_{7} e^{\kappa h_{t}}\left(C_{7}>0\right.$, see also (2.17) in Faggionato, 2009) and $\mathbb{E}\left(\left(\Delta m_{1}-\mathbb{E}\left(\Delta m_{1}\right)\right)^{4}\right) \sim C_{8} e^{4 \kappa h_{t}}\left(C_{8}>0\right)$, which yields as $t \rightarrow+\infty$ and $k \rightarrow+\infty$,

$$
\mathbb{E}\left[\left(m_{k} / k-\mathbb{E}\left(\Delta m_{1}\right)\right)^{4}\right] \sim C_{8} e^{4 \kappa h_{t}} / k^{2} .
$$

These facts allow us to write by a Markov inequality that

$$
\begin{aligned}
& \mathbb{P}\left[\left|m_{N_{t}}-\mathbb{E}\left(\Delta m_{1}\right) N_{t}\right|>\varepsilon \mathbb{E}\left(\Delta m_{1}\right) N_{t}\right] \\
\leq & \sum_{j \geq e^{(1-\varepsilon) \kappa \phi(t)}} \mathbb{P}\left[\left|m_{j}-\mathbb{E}\left(\Delta m_{1}\right) j\right|>\varepsilon \mathbb{E}\left(\Delta m_{1}\right) j\right]+o(1) \\
\leq & \sum_{j \geq e^{(1-\varepsilon) \kappa \phi(t)}} \frac{2 C_{8}\left(C_{7}\right)^{-4}}{\varepsilon^{4} j^{2}}+o(1) \\
\leq & C_{+} \varepsilon^{-4} e^{-(1-\varepsilon) \kappa \phi(t)}+o(1) .
\end{aligned}
$$

This yields that $\left\{\left|m_{N_{t}}-\mathbb{E}\left(\Delta m_{1}\right) N_{t}\right| \leq \varepsilon \mathbb{E}\left(\Delta m_{1}\right) N_{t}\right\}$ as well as (with a similar computation) $\left\{\left|m_{N_{t}^{*}}-\mathbb{E}\left(\Delta m_{1}\right) N_{t}^{*}\right| \leq \varepsilon \mathbb{E}\left(\Delta m_{1}\right) N_{t}^{*}\right\}$ are realized with a probability close to one.

Now including these events in the probability in (5.45), eventually enlarging $v(\varepsilon, t)$ we get

$$
\mathbb{P}\left[\frac{N_{t}^{*}}{N_{t}} \leq x \frac{(1-\varepsilon)^{2}}{(1+\varepsilon)^{2}}\right]-v(\varepsilon, t) \leq \mathbb{P}\left[\frac{F_{t}^{*}}{X(t)} \leq x\right] \leq \mathbb{P}\left[\frac{N_{t}^{*}}{N_{t}} \leq x \frac{(1+\varepsilon)^{2}}{(1-\varepsilon)^{2}}\right]+v(\varepsilon, t) .
$$

Notice that the random variables involved now $\left(N_{t}^{*}\right.$ and $\left.N_{t}\right)$ only depend of what happens in the bottom of the $h_{t}$-valleys, and we have to deal with

$$
\mathbb{P}\left[\frac{N_{t}^{*}}{N_{t}} \leq y\right]=\mathbb{P}\left[N_{t}^{*}=N_{t}\right] \mathbb{1}_{\{y=1\}}+\mathbb{P}\left[\frac{N_{t}^{*}}{N_{t}} \leq y, N_{t}^{*}<N_{t}\right] \mathbb{1}_{\{y \leq 1\}}+\mathbb{1}_{\{y>1\}},
$$

for any $y>0$. We are now interested in the limit when $t$ goes to infinity of the above two probabilities. We first use the same lines as for the proof of Section 5.1, that is to say we give a lower and an upper bound of this probability involving the i.i.d. sequences $\left(\ell_{j}, j\right)$ and $\left(\mathcal{H}_{j}, j\right)$. In the same way we have obtained Proposition 5.1 , we then have for any $\varepsilon>0$ and large $t$,

$$
\tilde{\mathcal{P}}-v(\varepsilon, t) \leq \mathbb{P}\left(N_{t}^{*}=N_{t}\right) \leq \tilde{\mathcal{P}}+v(\varepsilon, t)
$$

with

$$
\tilde{\mathcal{P}}:=\mathbb{P}\left[\left(1-\overline{\mathcal{H}}_{\mathcal{N}_{t}^{2 \varepsilon}-1}\right) \frac{\bar{\ell}_{\mathcal{N}_{t}^{2 \varepsilon}}-\bar{\ell}_{\mathcal{N}_{t}^{2 \varepsilon}-1}}{\left(\overline{\mathcal{H}}_{\mathcal{N}_{t}^{2 \varepsilon}}-\overline{\mathcal{H}}_{\mathcal{N}_{t}^{2 \varepsilon}-1}\right)}>\max _{1 \leq j \leq \mathcal{N}_{t}^{2 \varepsilon}-1} \frac{\ell_{j}}{t}\right]
$$

recall that $\overline{\mathcal{H}}_{k}=Y_{2}\left(k e^{-\kappa \phi(t)}\right)=\frac{1}{t} \sum_{i=1}^{k} \mathcal{H}_{i}, \bar{\ell}_{k}=Y_{1}\left(k e^{-\kappa \phi(t)}\right)=\frac{1}{t} \sum_{i=1}^{k} \ell_{i}, \mathcal{N}_{t}^{2 \varepsilon}:=$ $\inf \left\{m \geq 1, \overline{\mathcal{H}}_{m}>1-2 \varepsilon\right\}$, and $v$ is a positive function such that $\lim _{t \rightarrow+\infty} v(\varepsilon, t) \leq$ 
const $\times \varepsilon^{\kappa \wedge(1-\kappa)}$ with an eventually larger const than in Proposition 5.1. In the same way, for any $y>0, \varepsilon>0$ and $t$ large enough,

$$
\overline{\mathcal{P}}_{1}^{-}-v(\varepsilon, t) \leq \mathbb{P}\left[\frac{N_{t}^{*}}{N_{t}} \leq y, N_{t}^{*}<N_{t}\right] \mathbb{1}_{y \leq 1} \leq \overline{\mathcal{P}}_{1}^{+}+v(\varepsilon, t)
$$

where

$$
\begin{aligned}
& \tilde{\mathcal{P}}_{1}^{ \pm} \\
& :=\mathbb{P}\left[\mathcal{N}_{t}^{*} / \mathcal{N}_{t}^{2 \varepsilon} \leq y \pm \varepsilon,\left(1-\overline{\mathcal{H}}_{\mathcal{N}_{t}^{2 \varepsilon}-1}\right) \frac{\bar{\ell}_{\mathcal{N}_{t}^{2 \varepsilon}}-\bar{\ell}_{\mathcal{N}_{t}^{2 \varepsilon}-1}}{\left(\overline{\mathcal{H}}_{\mathcal{N}_{t}^{2 \varepsilon}}-\overline{\mathcal{H}}_{\mathcal{N}_{t}^{2 \varepsilon}-1}\right)} \leq \max _{1 \leq j \leq \mathcal{N}_{t}^{2 \varepsilon}-1} \frac{\ell_{j}}{t}\right] \mathbb{1}_{y \leq 1},
\end{aligned}
$$

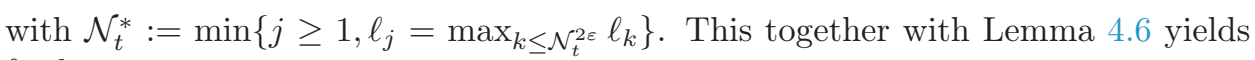
for large $t$,

$$
\left|\mathbb{P}\left[N_{t}^{*}=N_{t}\right]-\mathbb{P}\left[\mathcal{I}_{1}<\mathcal{I}_{2}\right]\right| \leq \lim _{t \rightarrow+\infty} v(\varepsilon, t)+o(1)
$$

and

$$
\begin{aligned}
& \leq\left|\mathbb{P}\left[\frac{N_{t}^{*} e^{-\kappa \phi(t)}}{N_{t} e^{-\kappa \phi(t)}} \leq y, N_{t}^{*}<N_{t}\right]-\mathbb{P}\left[\frac{F^{*}\left(\mathcal{Y}_{1}, \mathcal{Y}_{2}\right)}{\mathcal{Y}_{2}^{-1}(1)} \leq y, \mathcal{I}_{1} \geq \mathcal{I}_{2}\right]\right| \\
& \leq \lim _{t \rightarrow+\infty} v(\varepsilon, t),+o(1),
\end{aligned}
$$

where $F^{*}$ is defined at the beginning of Section 4.3. Replacing $y$ by $x \frac{(1-\varepsilon)^{2}}{(1+\varepsilon)^{2}}$ for the lower bound and by $x \frac{(1+\varepsilon)^{2}}{(1-\varepsilon)^{2}}$ for the upper bound and taking the limit when $t$ goes to infinity and then $\varepsilon \rightarrow 0$ we obtain for $0<x<1$,

$$
\lim _{t \rightarrow+\infty} \mathbb{P}\left[\frac{N_{t}^{*}}{N_{t}} \leq x\right]=\mathbb{P}\left[\frac{F^{*}\left(\mathcal{Y}_{1}, \mathcal{Y}_{2}\right)}{\mathcal{Y}_{2}^{-1}(1)} \leq x, \mathcal{I}_{1} \geq \mathcal{I}_{2}\right]
$$

To finish the proof of the last result of Theorem 1.5 we finally have to prove Lemma 5.5 below.

Lemma 5.5. The random variable $\frac{F^{*}\left(\mathcal{Y}_{1}, \mathcal{Y}_{2}\right)}{\mathcal{Y}_{2}^{-1}(1)}$ follows a uniform law $U_{[0,1]}$ and is independent of the couple $\left(\mathcal{I}_{1}, \mathcal{I}_{2}\right)$.

Proof: For any $s>0$, let $\mathcal{G}_{1}(s):=\inf \left\{u \leq s, \mathcal{Y}_{1}(u)-\mathcal{Y}_{1}(u-)=\mathcal{Y}_{1}^{\sharp}(s)\right\}$. The fact that for every $s>0, \mathcal{G}_{1}(s) / s$ follows a uniform distribution is basic. Since the independence that we seek is specific we give some details.

The process of the jumps of $\left(\mathcal{Y}_{1}, \mathcal{Y}_{2}\right)$ in $[0, s]$ is a Poisson point process in $[0, s] \times$ $\left(\mathbb{R}_{+}\right)^{2}$ (the coordinate in $[0, s]$ for the instant when the jump occurs and the other coordinate for the jump) with intensity measure $\lambda \times \nu$ where $\lambda$ is the Lebesgue measure on $[0, s]$ and $\nu$, as defined in the introduction, is the Lévy measure of $\left(\mathcal{Y}_{1}, \mathcal{Y}_{2}\right)$. Let us give a particular construction of the process $\left(\mathcal{Y}_{1}, \mathcal{Y}_{2}\right)$ on $[0, s]$ :

Let $\left(P_{n}\right)_{n \geq 1}$ be a countable partition of $\left(\mathbb{R}_{+}\right)^{2}$ by Borelian sets such that $\forall n \geq$ $1,0<\nu\left(P_{n}\right)<+\infty$. For each $n$ we define an i.i.d. sequence $\left(S_{k}^{n}\right)_{k \geq 1}$ of random variables in $\left(\mathbb{R}_{+}\right)^{2}$, an i.i.d. sequence $\left(U_{k}^{n}\right)_{k \geq 1}$ of random variables in $[0, s]$ and a random variable $T_{n}$ such that

- $\forall n \geq 1, S_{1}^{n} \sim \nu\left(. \cap P_{n}\right) / \nu\left(P_{n}\right), U_{1}^{n} \sim U_{[0, s]}, T_{n} \sim \mathcal{P}\left(s \nu\left(P_{n}\right)\right)$,

- For any $n \geq 1$, the variables $\left(S_{k}^{n}\right)_{k \geq 1},\left(U_{k}^{n}\right)_{k \geq 1}$ and $T_{n}$ are independent,

- The triplets $\left(\left(S_{k}^{n}\right)_{k \geq 1},\left(U_{k}^{n}\right)_{k \geq 1}, T_{n}\right)_{n \geq 1}$ are independent, 
where $U$ stands for uniform and $\mathcal{P}($.$) for Poisson distribution. We know that the$ random set

$$
\mathcal{S}_{n}:=\left\{\left(U_{k}^{n}, S_{k}^{n}\right), n \geq 1,1 \leq k \leq T_{n}\right\}
$$

is a Poisson point process in $[0, s] \times\left(\mathbb{R}_{+}\right)^{2}$ with intensity measure $\lambda \times \nu$. Since $\left(\mathcal{Y}_{1}, \mathcal{Y}_{2}\right)$ is pure jump, its restriction to $[0, s]$ is equal in law to the process $\left(\mathcal{Z}_{1}, \mathcal{Z}_{2}\right)$ defined by

$$
\forall r \in[0, s], \quad\left(\mathcal{Z}_{1}, \mathcal{Z}_{2}\right)(r)=\sum_{n \geq 1,1 \leq k \leq T_{n}} S_{k}^{n} \mathbb{1}_{U_{k}^{n} \leq r}
$$

In particular, with $\pi_{i}\left(x_{1}, x_{2}\right):=x_{i}$ for $\left(x_{1}, x_{2}\right) \in \mathbb{R}^{2}, i \in\{1,2\}$ and $\mathcal{G}_{1}^{\mathcal{Z}}(s):=$ $\inf \left\{u \leq s, \mathcal{Z}_{1}(u)-\mathcal{Z}_{1}\left(u^{-}\right)=\mathcal{Z}_{1}^{\sharp}(s)\right\} \stackrel{\text { I }}{=} \mathcal{G}_{1}(s)$, we have

$$
\begin{aligned}
\mathcal{Z}_{1}^{\sharp}(s) & =\max \left\{\pi_{1}\left(S_{k}^{n}\right), n \geq 1,1 \leq k \leq T_{n}\right\}, \\
\mathcal{G}_{1}^{\mathcal{Z}}(s) & =\inf \left\{U_{k}^{n}, n \geq 1,1 \leq k \leq T_{n}, \pi_{1}\left(S_{k}^{n}\right)=\mathcal{Z}_{1}^{\sharp}(s)\right\}, \\
\mathcal{Z}_{1}(s) & =\sum_{n \geq 1,1 \leq k \leq T_{n}} \pi_{1}\left(S_{k}^{n}\right), \quad \mathcal{Z}_{2}(s)=\sum_{n \geq 1,1 \leq k \leq T_{n}} \pi_{2}\left(S_{k}^{n}\right) .
\end{aligned}
$$

We thus have that $\mathcal{G}_{1}(s) / s \stackrel{\text { II }}{=} U_{[0,1]}$ and it is independent from $\left(\mathcal{Y}_{1}^{\sharp}(s), \mathcal{Y}_{1}(s), \mathcal{Y}_{2}(s)\right)$ and from the sigma-field $\sigma\left(\left(\mathcal{Y}_{1}, \mathcal{Y}_{2}\right)(t+s)-\left(\mathcal{Y}_{1}, \mathcal{Y}_{2}\right)(s), t \geq 0\right)$.

We now have to replace $s$ by $\mathcal{Y}_{2}^{-1}(1)$. For that we can consider for example the dyadic approximations of $\mathcal{Y}_{2}^{-1}(1)$, that is, $\left(t_{n}:=\max \left\{k \in \mathbb{N}, \frac{k}{2^{n}}<\mathcal{Y}_{2}^{-1}(1)\right\}, n\right)$. Then, partitioning on the values of $t_{n}$, using the independence we just proved and the fact that $\mathcal{G}_{1}(s) / s$ follows a uniform distribution on $[0,1]$ we get that $\mathcal{G}_{1}\left(t_{n}\right) / t_{n}$ follows a uniform distribution on $[0,1]$ and is independent from

$$
\left(\left(\mathcal{Y}_{1}^{\sharp}\left(t_{n}\right), \mathcal{Y}_{2}\left(t_{n}\right), \mathcal{Y}_{1}\left(t_{n}+2^{-n}\right)-\mathcal{Y}_{1}\left(t_{n}\right), \mathcal{Y}_{2}\left(t_{n}+2^{-n}\right)-\mathcal{Y}_{2}\left(t_{n}\right)\right)\right.
$$

We let $n$ goes to infinity, $t_{n}$ converges almost surely to $\mathcal{Y}_{2}^{-1}(1)$ from below. As a consequence, $\mathcal{G}_{1}\left(t_{n}\right) / t_{n}$ converges almost surely to $\frac{F^{*}\left(\mathcal{Y}_{1}, \mathcal{Y}_{2}\right)}{\mathcal{Y}_{2}^{-1}(1)}$ while the quadruple in (5.46) converges almost surely to

$$
\begin{aligned}
\left(\mathcal{Y}_{1}^{\sharp}\left(\mathcal{Y}_{2}^{-1}(1)-\right),\right. & \mathcal{Y}_{2}\left(\mathcal{Y}_{2}^{-1}(1)-\right), \\
& \left.\mathcal{Y}_{1}\left(\mathcal{Y}_{2}^{-1}(1)\right)-\mathcal{Y}_{1}\left(\mathcal{Y}_{2}^{-1}(1)-\right), \mathcal{Y}_{2}\left(\mathcal{Y}_{2}^{-1}(1)\right)-\mathcal{Y}_{2}\left(\mathcal{Y}_{2}^{-1}(1)-\right)\right) .
\end{aligned}
$$

As a consequence, $\frac{F^{*}\left(\mathcal{Y}_{1}, \mathcal{Y}_{2}\right)}{\mathcal{Y}_{2}^{-1}(1)}$ follows a uniform distribution on $[0,1]$ and is independent from the above quadruple for which $\left(\mathcal{I}_{1}, \mathcal{I}_{2}\right)$ is a measurable function, this yields the lemma.

\section{Results and additional arguments from the paper Andreoletti and} Devulder (2015)

6.1. Some estimates on the diffusion $X$. The first lemma below gives the right normalisation in law of the number of $h_{t}$-valleys visited by $X$ before time $t$.

Lemma 6.1 (number of visited $h_{t}$-valleys). Assume that $0<\kappa<1$. Then, under the annealed law $\mathbb{P}, N_{t} e^{-\kappa \phi(t)} \rightarrow_{t \rightarrow+\infty} \mathcal{N}$ in law. The law of $\mathcal{N}$ is determined by its Laplace transform:

$$
\forall u>0, \quad \mathbb{E}\left(e^{-u \mathcal{N}}\right)=\sum_{j=0}^{+\infty} \frac{1}{\Gamma(\kappa j+1)}\left(\frac{-u}{\mathcal{C}_{\kappa}}\right)^{j},
$$


where $\mathcal{C}_{\kappa}$ is a positive constant. Moreover $\mathbb{P}\left(N_{t}>n_{t}\right) \leq e^{-\phi(t)}$.

Proof: The convergence in distribution is exactly Proposition 1.6 of Andreoletti and Devulder (2015). For the second fact we have $\mathbb{P}\left(N_{t} \geq n_{t}\right) \leq \mathbb{P}\left(\tilde{N}_{t} \geq n_{t}\right)+$ $\mathbb{P}\left(\overline{\mathcal{V}}_{t}\right) \leq \mathbb{P}\left(\tilde{N}_{t} \geq n_{t}\right)+e^{[-\kappa / 2+o(1)] h_{t}}$ by Lemma 2.2, with $\tilde{N}_{t}:=\max \left\{j \geq 1, \tilde{m}_{j} \leq\right.$ $\left.\sup _{s \leq t} X(s)\right\}$. Then equation (5.3) in Andreoletti and Devulder (2015) gives $\mathbb{P}\left(\tilde{N}_{t} \geq\right.$ $\left.n_{t}\right) \leq \exp (-2 \phi(t))$, which yields the result.

The lemma below deals with the renewal structure we speak about on the introduction, and the consequence on the hitting time $H\left(m_{N_{t}}\right)$ of the ultimate $h_{t}$-valley visited by $X$ before time $t$.

Lemma 6.2. Assume $0<\kappa<1$ and $0<\delta<\inf \left\{2 / 27, \kappa^{2} / 2\right\}$. For $t>0$, let $\mu_{t}$ be the positive measure on $\mathbb{R}_{+}$such that

$$
\forall x \geq 0, \quad \mu_{t}([0, x]):=e^{-\kappa \phi(t)} \sum_{j=1}^{n_{t}} \mathbb{P}\left(\overline{\mathcal{H}}_{j} \leq x\right) .
$$

Recall that for any $k, \overline{\mathcal{H}}_{k}:=\sum_{j=1}^{k} \mathcal{H}_{j} / t$, and $\mathcal{H}_{1}=R_{1} S_{1} \mathbf{e}_{\mathbf{1}}$ is defined in Proposition 3.5. Then, $\left(\mu_{t}\right)_{t}$ converges vaguely as $t \rightarrow+\infty$ to $\mu$ defined by

$$
\mathrm{d} \mu(x):=\left(\mathcal{C}_{\kappa} \Gamma(\kappa)\right)^{-1} x^{\kappa-1} \mathbb{1}_{(0,+\infty)}(x) \mathrm{d} x,
$$

with $\mathcal{C}_{\kappa}$ is the same constant as in Lemma 6.1. For $0 \leq r<s \leq 1$,

$$
\lim _{t \rightarrow+\infty} \mathbb{P}\left(1-s \leq \frac{H\left(m_{N_{t}}\right)}{t} \leq 1-r\right)=\frac{\sin (\pi \kappa)}{\pi} \int_{1-s}^{1-r} x^{\kappa-1}(1-x)^{-\kappa} \mathrm{d} x .
$$

Proof: The first part of the above lemma is very close to Lemma 5.1 of Andreoletti and Devulder (2015), indeed Proposition 3.5 gives the proximity between the random variables $\left(U_{i}, i \leq n_{t}\right)$ and the random variables $\left(\mathcal{H}_{i}, i \leq n_{t}\right)$, moreover an important preliminary result in Andreoletti and Devulder (2015) (Proposition 4.1) states that $e^{\kappa \phi(t)}\left(1-\mathbb{E}\left(e^{-\lambda U_{1} / t}\right)\right)=\mathcal{C}_{\kappa} \lambda^{\kappa}+o(1)$ for large $t$. So we also know that

$$
e^{\kappa \phi(t)}\left(1-\mathbb{E}\left(e^{-\lambda \mathcal{H}_{1} / t}\right)\right)=\mathcal{C}_{\kappa} \lambda^{\kappa}+o(1),
$$

notice that this result could also be deduced from (4.2) with the help of a Tauberian theorem. Then by independence of the random variables $\mathcal{H}_{j}$ and the fact that they are i.d., for any $\lambda>0$

$$
\int_{0}^{+\infty} e^{-\lambda x} \mathrm{~d} \mu_{t}(x)=\frac{1}{e^{\kappa \phi(t)}} \sum_{j=1}^{n_{t}}\left(\mathbb{E}\left(e^{-\lambda \frac{\mathcal{H}_{1}}{t}}\right)\right)^{j}
$$

By (6.3) as $n_{t} e^{-\kappa \phi(t)} \rightarrow_{t \rightarrow+\infty}+\infty,\left[\mathbb{E}\left(e^{-\lambda \mathcal{H}_{1} / t}\right)\right]^{n_{t}+1}=o(1)$. Hence, we get as $t \rightarrow+\infty$, again by 6.3

$$
\begin{aligned}
\int_{0}^{+\infty} e^{-\lambda x} \mathrm{~d} \mu_{t}(x) & =\frac{e^{-\kappa \phi(t)}(1+o(1))}{1-\mathbb{E}\left(e^{-\lambda \mathcal{H}_{1} / t}\right)}+o(1)=\frac{1}{\mathcal{C}_{\kappa} \lambda^{\kappa}}+o(1) \\
& =\int_{0}^{+\infty} \frac{e^{-\lambda x} x^{\kappa-1}}{\mathcal{C}_{\kappa} \Gamma(\kappa)} \mathrm{d} x+o(1)
\end{aligned}
$$

which gives the vague convergence of measure $\left(\mu_{t}\right)_{t}$. Also (6.2) is equation (1.2) of Corollary 1.5 in Andreoletti and Devulder (2015). 
In Lemma 6.3 below, we approximate $\tilde{h}_{j}$, the exit time of $h_{t}$-valley number $j$ (if $X$ leaves it on the right), by a product of 3 simpler random variables. To this aim, we recall that with the notation of Lemma 3.6 and of its proof, for each $1 \leq j \leq n_{t}$, $\tilde{R}_{j}=\int_{\tilde{\tau}_{j}^{-}\left(h_{t} / 2\right)}^{\tilde{\tau}_{j}\left(h_{t} / 2\right)} e^{-\tilde{V}^{(j)}(x)} \mathrm{d} x$, and $A^{j}(u)=\int_{\tilde{m}_{j}}^{u} e^{\tilde{V}^{(j)}(x)} \mathrm{d} x, u \in \mathbb{R}$. Moreover, for some independent Brownian motions $B^{j}, 1 \leq j \leq n_{t}$, independent of $W_{\kappa}$,

$$
\begin{aligned}
\tilde{h}_{j} & =\int_{\tilde{L}_{j}^{-}}^{\tilde{L}_{j}} e^{-\tilde{V}^{(j)}(u)} \mathcal{L}_{B^{j}}\left[\tau^{B^{j}}\left(A^{j}\left(\tilde{L}_{j}\right)\right), A^{j}(u)\right] \mathrm{d} u, \\
\mathbf{e}_{j} & =\mathcal{L}_{B^{j}}\left[\tau^{B^{j}}\left(A^{j}\left(\tilde{L}_{j}\right)\right), 0\right] / A^{j}\left(\tilde{L}_{j}\right) .
\end{aligned}
$$

Lemma 6.3. Let $0<\varepsilon<\inf \left\{2 / 27, \kappa^{2} / 2\right\}$. For large $t$, we have for every $1 \leq j \leq$ $n_{t}$,

$$
\mathbb{P}\left(\left|\tilde{h}_{j}-A^{j}\left(\tilde{L}_{j}\right) \mathbf{e}_{j} \tilde{R}_{j}\right|>2 e^{-(1-3 \varepsilon) h_{t} / 6} A^{j}\left(\tilde{L}_{j}\right) \mathbf{e}_{j} \tilde{R}_{j}\right) \leq C_{+} e^{-\left(c_{-}\right) \varepsilon h_{t}}
$$

Proof: We first notice that $\left(\tilde{h}_{j}, A^{j}\left(\tilde{L}_{j}\right), \mathbf{e}_{j}, \tilde{R}_{j}\right)$ is measurable with respect to the $\sigma$-field generated by $\left(\tilde{V}^{(j)}\left(x+\tilde{L}_{j-1}^{+}\right), 0 \leq x \leq \tilde{L}_{j}^{+}-\tilde{L}_{j-1}^{+}\right)$and $B^{j}$, so, thanks to the second fact of Lemma 2.2, its law under $\mathbb{P}$ does not depend on $j$. Thus, the left hand side of (6.4) does not depend on $j$. Hence we just have to prove (6.4) for $j=2$.

This is actually already proved in Andreoletti and Devulder (2015), for which it is an important step. Indeed in this paper Andreoletti and Devulder (2015), our $A^{j}, \tilde{B}^{2}$ and $\tilde{h}_{2}$ are denoted respectively by $\tilde{A}_{j}, B$ and $\mathbf{U}$, as defined in Andreoletti and Devulder (2015, eq. (3.17) and (3.18)), and our $\tilde{R}_{2}$ and $\mathbf{e}_{2}$ by $\mathcal{I}^{-}$and $\mathbf{e}_{1}$, as defined in Andreoletti and Devulder (2015, after eq. (4.17)). Hence our (6.4) for $j=2$ is exactly Andreoletti and Devulder (2015, Lemma 4.7), which proves our lemma.

The proof of Andreoletti and Devulder (2015, Lemma 4.7) is quite technical, however we can give a simple heuristic in order for the present paper to be more self-contained. The idea of the proof of Andreoletti and Devulder (2015, Lemma $4.7)$ is that, loosely speaking, for $u$ close to $\tilde{m}_{j}$, that is for $u \in\left[\tilde{\tau}_{j}^{-}\left(h_{t} / 2\right), \tilde{\tau}_{j}\left(h_{t} / 2\right)\right]$, $\mathcal{L}_{B^{j}}\left[\tau^{B^{j}}\left(A^{j}\left(\tilde{L}_{j}\right)\right), A^{j}(u)\right]$ is nearly $\mathcal{L}_{B^{j}}\left[\tau^{B^{j}}\left(A^{j}\left(\tilde{L}_{j}\right)\right), 0\right]=A^{j}\left(\tilde{L}_{j}\right) \mathbf{e}_{\mathbf{j}}$, whereas for $u$ far from $\tilde{m}_{j}$, that is for $u \in\left[\tilde{L}_{j}^{-}, \tilde{L}_{j}\right]$ but $u \notin\left[\tilde{\tau}_{j}^{-}\left(h_{t} / 2\right), \tilde{\tau}_{j}\left(h_{t} / 2\right)\right], e^{-\tilde{V}^{(j)}(x)}$ is "nearly" 0 , with large probability. Finally, combining these heuristics gives $\tilde{h}_{j} \approx$ $A^{j}\left(\tilde{L}_{j}\right) \mathbf{e}_{\mathbf{j}} \tilde{R}_{j}$.

The following lemma is used to prove Lemma 3.6 and uses the notation of this lemma, and where the independent r.v. $G^{+}\left(h_{t} / 2, h_{t}\right), F_{1}^{+}\left(h_{t}\right), F_{2}^{-}\left(h_{t} / 2\right)$ and $F_{3}^{-}\left(h_{t} / 2\right)$ defined before Proposition 3.5.

Lemma 6.4. Assume $0<\delta<\inf \left\{2 / 27, \kappa^{2} / 2\right\}$. For large $t$, possibly on an enlarged

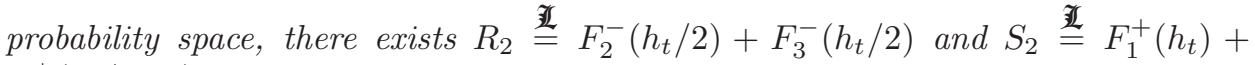
$G^{+}\left(h_{t} / 2, h_{t}\right)$, such that $R_{2}, S_{2}$ and $\mathbf{e}_{2}$ are independent and

$$
P\left(\left\{\left|\int_{\tilde{m}_{2}}^{\tilde{L}_{2}} e^{\tilde{V}^{(2)}(x)} \mathrm{d} x-S_{2}\right| \leq e^{-\left(d_{-}\right) h_{t}} S_{2}, \tilde{R}_{2}=R_{2}\right\}\right) \geq 1-e^{-\left(D_{-}\right) h_{t}},
$$

where $D_{-}>0$. 
Proof: Due to Andreoletti and Devulder (2015, Lemma 4.5) with its notation, we

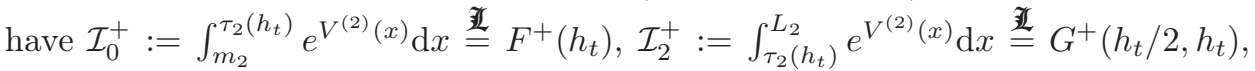
$\mathcal{I}_{1}^{-}:=\int_{m_{2}}^{\tau_{2}\left(h_{t} / 2\right)} e^{-V^{(2)}(x)} \mathrm{d} x \stackrel{\text { 亲 }}{=} F^{-}\left(h_{t} / 2\right)$ and finally $\mathcal{I}_{2}^{-}:=\int_{\tau_{2}^{-}\left(h_{t} / 2\right)}^{m_{2}} e^{-V^{(2)}(x)} \mathrm{d} x \stackrel{\text { II }}{=}$ $F^{-}\left(h_{t} / 2\right)$ with $L_{2}:=\inf \left\{x>\tau_{2}\left(h_{t}\right), V^{(2)}(x)=h_{t} / 2\right\}$. The problem is that $\mathcal{I}_{0}^{+}$is not independent of $\mathcal{I}_{1}^{-}$, so we would like to replace it by some $\mathcal{I}_{1}^{+} \stackrel{\text { II }}{=} \mathcal{I}_{0}^{+}$of it with better independence properties. It is proved in Andreoletti and Devulder (2015, at the top of page 32) that for large $t$, possibly in an enlarged probability space, there exists $\mathcal{I}_{1}^{+}$such that $\left|\mathcal{I}_{0}^{+}-\mathcal{I}_{1}^{+}\right| \leq e^{-(1-3 \delta) h_{t} / 2} \mathcal{I}_{1}^{+}$with probability greater than $1-4 e^{-\kappa \delta h_{t} / 2}$ and where $\mathcal{I}_{1}^{+} \stackrel{\text { IL }}{=} F^{+}\left(h_{t}\right)$ by Andreoletti and Devulder (2015, eq. $(4.35))$.

Let $S_{2}:=\mathcal{I}_{1}^{+}+\mathcal{I}_{2}^{+} \geq \mathcal{I}_{1}^{+}$. Notice that on $\mathcal{V}_{t}$, by Remark 2.3, $\tilde{R}_{2}=\mathcal{I}_{1}^{-}+\mathcal{I}_{2}^{-}=: R_{2}$ and $\int_{\tilde{m}_{2}}^{\tilde{L}_{2}} e^{\tilde{V}^{(2)}(x)} \mathrm{d} x=\int_{m_{2}}^{L_{2}} e^{V^{(2)}(x)} \mathrm{d} x=\mathcal{I}_{0}^{+}+\mathcal{I}_{2}^{+}$. The two previous inequalities give $\left|\int_{\tilde{m}_{2}}^{\tilde{L}_{2}} e^{\tilde{V}^{(2)}(x)} \mathrm{d} x-S_{2}\right|=\left|\mathcal{I}_{0}^{+}-\mathcal{I}_{1}^{+}\right| \leq e^{-(1-3 \delta) h_{t} / 2} S_{2}$ and $\tilde{R}_{2}=R_{2}$ with probability at least $1-5 e^{-\kappa \delta h_{t} / 2}$ thanks to Lemma 2.2. This proves (6.5).

Moreover, by Andreoletti and Devulder (2015, Prop. 4.4 (i)), $\mathcal{I}_{1}^{+}, \mathcal{I}_{2}^{+}, \mathcal{I}_{1}^{-}, \mathcal{I}_{2}^{-}$ and $\mathbf{e}_{2}$ (which is denoted by $\mathbf{e}_{1}$ in Andreoletti and Devulder, 2015) are independent. So, $\mathbf{e}_{2}, S_{2}=\mathcal{I}_{1}^{+}+\mathcal{I}_{2}^{+}$and $R_{2}=\mathcal{I}_{1}^{-}+\mathcal{I}_{2}^{-}$are independent, and $R_{2} \stackrel{\text { 近 }}{=} F_{2}^{-}\left(h_{t} / 2\right)+$ $F_{3}^{-}\left(h_{t} / 2\right)$ and $S_{2} \stackrel{\text { 至 }}{=} F_{1}^{+}\left(h_{t}\right)+G^{+}\left(h_{t} / 2, h_{t}\right)$.

The last lemma of this section tells that with large probability, the diffusion $X$ leaves every $h_{t}$-valley $\left[\tilde{L}_{j}^{-}, \tilde{L}_{j}\right], 1 \leq j \leq n_{t}$ from its right. Recall that $B^{j}$ is defined after (3.19).

Lemma 6.5. For large $t$, there exists $c_{-}>0$ such that

$$
\mathbb{P}\left[\cap_{j=1}^{n_{t}}\left\{\max _{u<\tilde{L}_{j}^{-}} \mathcal{L}_{B^{j}}\left[\tau^{B^{j}}\left(A^{j}\left(\tilde{L}_{j}\right)\right), A^{j}(u)\right]=0\right\}\right] \geq 1-e^{-\left(c_{-}\right) h_{t}} .
$$

Proof: (6.6) is essentially Lemma 3.2 in Andreoletti and Devulder (2015):

Indeed, recall the definition of $\mathcal{A}_{j}:=\left\{\max _{u<\tilde{L}_{j}^{-}} \mathcal{L}_{B^{j}}\left[\tau^{B^{j}}\left(A^{j}\left(\tilde{L}_{j}\right)\right), A^{j}(u)\right]=0\right\}$, we have $\cap_{j=1}^{n_{t}} \mathcal{A}_{j}=\cap_{j=1}^{n_{t}}\left\{H_{j}\left(\tilde{L}_{j}\right)<\left\{H_{j}\left(\tilde{L}_{j}^{-}\right)\right\}\right.$, with, for any $\tilde{L}_{j}^{-} \leq x \leq \tilde{L}_{j}, H_{j}(x)=$ $\inf \left\{s>0, B_{j}(s)=x\right\}$, with $B_{j}$ a Brownian motion. Therefore $\mathbb{P}^{W_{\kappa}}\left(\mathcal{A}_{j}\right)$ is equal to the probability $\mathbb{P}^{W_{\kappa}}\left(\overline{\mathcal{E}}_{j}\right)$ of Lemma 3.2 in Andreoletti and Devulder (2015). It is proved in this lemma see $(3.10)$ that for large $t, P\left(\mathcal{B}:=\left\{\mathbb{P}^{W_{\kappa}}\left(\overline{\mathcal{E}}_{j}\right) \leq e^{-(\kappa / 2) h_{t}}\right\}\right) \geq$ $1-3 e^{-\kappa \delta h_{t}}$, so we obtain $(6.6)$ as $\mathbb{P}\left(\overline{\mathcal{E}}_{j}\right) \leq E\left(\mathbb{P}^{W_{\kappa}}\left(\overline{\mathcal{E}}_{j}\right) \mathbb{1}_{\mathcal{B}}\right)+P(\overline{\mathcal{B}}) \leq e^{-c_{-} h_{t}} / n_{t}$, for $c_{-}>0$ small enough.

6.2. Some estimates on the potential $W_{\kappa}$ and its functionals.

We start this section with the Laplace transform of the important functional $\mathcal{R}_{\kappa}$ :

Lemma 6.6. Recall that $0<\kappa<1$. For any $\gamma>0$,

$$
E\left(e^{-\gamma \mathcal{R}_{\kappa}}\right)=\left(\frac{(2 \gamma)^{\kappa / 2}}{\kappa \Gamma(\kappa) I_{\kappa}(2 \sqrt{2 \gamma})}\right)^{2} .
$$

Moreover, $\mathcal{R}_{\kappa}$ admits moments of any positive order. 
Proof: $\int_{0}^{+\infty} e^{-W_{\kappa}^{\uparrow}(u)} \mathrm{d} u$ is the limit in law under $P$ of $\int_{0}^{\tau^{W_{\kappa}^{\uparrow}}(x)} e^{-W_{\kappa}^{\uparrow}(u)} \mathrm{d} u$ as $x \rightarrow$ $+\infty$. This limit is given by Andreoletti and Devulder (2015, Lemma 4.2), which proves (6.7). Note that in Andreoletti and Devulder (2015, Lemma 4.2), $W_{\kappa}^{\uparrow}$ is denoted by $R$, and $\int_{0}^{\tau_{\kappa}^{W_{\kappa}^{\uparrow}}(x)} e^{-W_{\kappa}^{\uparrow}(u)} \mathrm{d} u$ is denoted respectively by $F^{-}(x)$. Moreover the Laplace transform of $\mathcal{R}_{\kappa}$ is of class $C^{\infty}$ on a neighborhood of 0 since $x \mapsto$ $x^{\kappa} / I_{\kappa}(x)$ is $C^{\infty}$ on such a neighborhood (see e.g. Borodin and Salminen, $2002 \mathrm{p}$. 638). Therefore $\mathcal{R}_{\kappa}$ admits moments of any positive order.

The following Lemma is a series of estimates concerning the different coordinates of valleys.

Lemma 6.7. For $t$ large enough, for every $1 \leq i \leq n_{t}$,

$$
\begin{aligned}
& P\left(0<M_{0}<m_{1}\right) \leq C_{+} h_{t} e^{-\kappa h_{t}}, \\
& P\left(\tilde{\tau}_{i+1}^{*}\left(h_{t}\right) \neq \tilde{\tau}_{i+1}\left(h_{t}\right)\right) \leq C_{+} h_{t} e^{-\kappa h_{t}}, \\
& P\left(\inf _{\left[\tilde{\tau}_{i}^{-}\left(h_{t}^{+}\right), \tilde{\tau}_{i}^{-}\left(h_{t}\right)\right]} \tilde{V}^{(i)}<h_{t} / 2\right) \leq e^{-\kappa h_{t} / 8}, \\
& P\left(\tilde{L}_{i}^{+}-\tilde{L}_{i}^{-} \geq 40 h_{t}^{+} / \kappa\right) \leq e^{-\kappa h_{t} / 8}, \\
& P\left(\tilde{\tau}_{i}(h)-\tilde{m}_{i} \geq 8 h / \kappa\right) \leq C_{+} e^{-\kappa h /(2 \sqrt{2})}, \quad 0 \leq h \leq h_{t}, \quad \\
& P\left(\tilde{m}_{1} \leq r\right) \leq e^{r} \exp \left(\left(\kappa / 2-\sqrt{2+\kappa^{2} / 4}\right) h_{t}^{+}\right)=o(1), \quad \forall r=o\left(h_{t}^{+}\right) .
\end{aligned}
$$

Proof: (6.8) follows from eq. (2.8) of Andreoletti and Devulder (2015); (6.9) is eq. (3.41) of Andreoletti and Devulder (2015). (6.10) and (6.11) are respectively eq. (2.34) and (2.32) of Lemma 2.7 of Andreoletti and Devulder (2015). Moreover, (6.12) is eq. (2.22) of the same reference. For (6.13), we know from definitions in (2.3) that $\tilde{m}_{1} \geq \tilde{L}_{1}^{\sharp}=\tau^{W_{\kappa}}\left(-h_{t}^{+}\right)$, where $\tau^{W_{\kappa}}\left(-h_{t}^{+}\right)$is the first positive time the drifted Brownian motion $W_{\kappa}$ reaches $-h_{t}$. Using a Markov inequality together with (2.0.1) page 295 of Borodin and Salminen (2002) we obtain $P\left(\tau^{W_{\kappa}}\left(-h_{t}^{+}\right) \leq r\right)=$ $P\left(e^{-\tau^{W_{\kappa}}\left(-h_{t}^{+}\right)} \geq e^{-r}\right) \leq e^{r} e^{\left(\kappa / 2-\sqrt{2+\kappa^{2} / 4}\right) h_{t}^{+}}$, which is exactly (6.13).

The lemma below deals with two functionals involving coordinates far from the bottom $\tilde{m}_{1}$ of the first visited $h_{t}$-valley $\left[\tilde{L}_{1}^{-}, \tilde{L}_{1}\right]$.

Lemma 6.8. There exists $c_{-}>0$ such that for any $\varepsilon>0$ and $t$ large enough,

$$
\begin{gathered}
P\left(\int_{\tilde{\tau}_{1}\left(h_{t} / 2\right)}^{\tilde{L}_{1}} e^{-\tilde{V}^{(1)}(x)} \mathrm{d} x \leq C_{+} h_{t}^{2} e^{-(1-\varepsilon) h_{t} / 2}\right) \geq 1-e^{-\left(c_{-}\right) \varepsilon h_{t}}, \\
P\left(\int_{\tilde{L}_{1}^{-}}^{\tilde{\tau}_{1}^{-}\left(h_{t} / 2\right)} e^{-\tilde{V}^{(1)}(x)} \mathrm{d} x \leq C_{+} h_{t}^{2} e^{-(1-\varepsilon) h_{t} / 2}\right) \geq 1-e^{-\left(c_{-}\right) \varepsilon h_{t}} .
\end{gathered}
$$

Proof: The proof is inspired from steps 1 and 2 of Lemma 4.7 of Andreoletti and Devulder (2015). For the first integral, let

$$
\left.\mathcal{A}_{1}:=\left\{\inf \left[\tilde{\tau}_{1}\left(h_{t} / 2\right)\right), \tilde{\tau}_{1}\left(h_{t}\right)\right] \tilde{V}^{(1)}>(1-\varepsilon) h_{t} / 2\right\}, \quad \mathcal{A}_{2}:=\left\{\tilde{L}_{1}^{+}-\tilde{L}_{1}^{-} \leq 40 h_{t}^{+} / \kappa\right\} .
$$

We have on $\mathcal{A}_{1} \cap \mathcal{A}_{2}$,

$$
\left.\int_{\left.\tilde{\tau}_{1}\left(h_{t} / 2\right)\right)}^{\tilde{L}_{1}} e^{-\tilde{V}^{(1)}(u)} \mathrm{d} u \leq e^{-(1-\varepsilon) h_{t} / 2}\left[\tilde{L}_{1}-\tilde{\tau}_{1}\left(h_{t} / 2\right)\right)\right] \leq \frac{40 h_{t}^{+} h_{t}}{\kappa} e^{-(1-\varepsilon) h_{t} / 2} .
$$


Now, Fact 2.1, equation (7.3) with $\alpha=1 / 2, \gamma=(1-\varepsilon) / 2$ and $\omega=1$, and Lemma 2.2 give

$$
P\left(\overline{\mathcal{A}_{1}}\right) \leq P\left[\inf _{\left[\tau_{1}\left(h_{t} / 2\right), \tau_{1}\left(h_{t}\right)\right]} V^{(1)} \leq(1-\varepsilon) h_{t} / 2, \mathcal{V}_{t}\right]+P\left(\overline{\mathcal{V}}_{t}\right) \leq 3 e^{-\kappa \varepsilon h_{t} / 2} .
$$

Moreover, $P\left(\overline{\mathcal{A}_{2}}\right) \leq e^{-\kappa h_{t} / 8} \leq e^{-\kappa \varepsilon h_{t} / 2}$ by (6.11) since we can take $\varepsilon<1 / 4$. The second inequality, can be proved similarly.

Lemma 6.9. Recall that for $h>0, \beta_{0}(h):=E\left(\int_{0}^{\tau_{1}^{*}(h)} e^{W_{\kappa}(u)} \mathrm{d} u\right)$, with $\tau_{1}^{*}(h):=$ $\inf \left\{u \geq 0, W_{\kappa}(u)-\inf _{[0, u]} W_{\kappa} \geq h\right\}$. For large $h$,

$$
\beta_{0}(h) \leq C_{+} e^{(1-\kappa) h} .
$$

Proof: (6.15) is Andreoletti and Devulder (2015, eq. (3.38)), since in Andreoletti and Devulder (2015), $\beta_{0}(h)$ is defined at the top of page 23 and $\tau_{1}^{*}(h)$ in its Lemma 3.6 .

\section{Appendix}

7.1. Some estimates for Brownian motion, Bessel processes, $W_{\kappa}^{\uparrow}$ and their functionals. We provide in this section some known formulas for some processes that appear in our study. The first lemma is about Laplace transforms of the exponential functionals defined in (1.6) and (1.7). Its proof can be found in Andreoletti and Devulder (2015, Lemma 4.2). Recall that $C_{+}$(respectively $c_{-}$) is a positive constant that is as large (resp. small) as needed.

Lemma 7.1. There exist $C_{9}>0, M>0$ and $\eta_{1} \in(0,1)$ such that $\forall y>M, \forall \gamma \in$ $\left(0, \eta_{1}\right]$,

$$
\begin{gathered}
\left|E\left(e^{-\gamma F^{+}(y) / e^{y}}\right)-[1-2 \gamma /(\kappa+1)]\right| \leq C_{9} \max \left(e^{-\kappa y}, \gamma^{3 / 2}\right) \\
\left|E\left(e^{-\gamma G^{+}(y / 2, y) / e^{y}}\right)-\left[1-\Gamma(1-\kappa)(2 \gamma)^{\kappa} / \Gamma(1+\kappa)\right]\right| \leq C_{9} \max \left(\gamma^{\kappa} e^{-\kappa y / 2}, \gamma\right) .
\end{gathered}
$$

Moreover, there exists $C_{10}>0$ such that for all $y>0, E\left(F^{+}(y) / e^{y}\right) \leq C_{10}$.

Recall that $W_{\kappa}^{\uparrow}$ is a $(-\kappa / 2)$-drifted Brownian motion $W_{\kappa}$ Doob-conditioned to stay positive (see above (1.6)). We have,

Lemma 7.2. Let $0<\gamma<\alpha<\omega$. For all $h$ large enough, we have

$$
\begin{aligned}
P^{\alpha h}\left(\tau^{W_{\kappa}^{\uparrow}}(\gamma h)<\tau_{\kappa}^{W_{\kappa}^{\uparrow}}(\omega h)\right) & \leq 2 e^{-\kappa(\alpha-\gamma) h}, \\
P\left(\tau^{W_{\kappa}^{\uparrow}}(\omega h)-\tau^{W_{\kappa}^{\uparrow}}(\alpha h) \leq 1\right) & \leq 4 e^{-[(\omega-\alpha) h]^{2} / 3}, \\
P\left(\tau_{\kappa}^{W_{\kappa}^{\uparrow}}(h)>8 h / \kappa\right) & \leq C_{+} e^{-\kappa h /(2 \sqrt{2})}, \\
P\left(\tau^{W_{\kappa}^{\uparrow}}(h) \leq h\right) & \leq C_{+} e^{-\left(c_{-}\right) h}, \\
P\left(\tau^{W_{\kappa}^{\uparrow}}(\gamma h) \leq 1\right) & \leq C_{+} e^{-\left(c_{-}\right)[\gamma h]^{2}},
\end{aligned}
$$

where $P^{\alpha h}$ denotes the law of $W_{\kappa}^{\uparrow}$ starting from $\alpha h$. Moreover the first inequality is still true if $\omega$ is a function of $h$ such that $\lim _{h \rightarrow+\infty} \omega(h)=+\infty$. 
Proof: The first 3 inequalities come from Andreoletti and Devulder (2015, Lemma 2.6). The fact that, in (7.3), $\omega$ can actually be taken as a function of $h$ comes directly from eq. (2.31) of Andreoletti and Devulder (2015), which shows that the right hand side of (7.3) is equivalent to $e^{-\kappa(\alpha-\gamma) h}$ as $h \rightarrow+\infty$ if $w=w(h) \rightarrow_{h \rightarrow+\infty}$ $+\infty$. (7.7) is a consequence of (7.4) with $\omega=\gamma$ and $\alpha=\gamma / 2$. We turn to (7.6). By Andreoletti and Devulder (2015, eq. (2.7)) and Fact 2.1, coming from Faggionato (2009), $E\left(e^{-\alpha \tau_{\kappa}^{W_{\kappa}^{\uparrow}}(h)}\right) \sim_{h \rightarrow+\infty}$ const. $e^{h\left(\kappa / 2-\sqrt{2 \alpha+\kappa^{2} / 4}\right)}$, in particular for $\alpha=1-\kappa$. Then a Markov inequality for $P\left(e^{-\alpha \tau_{\kappa}^{W_{\kappa}^{\uparrow}}(h)}>e^{-\alpha h}\right)$ proves (7.6) since $1-\kappa / 2-\sqrt{2(1-\kappa)+\kappa^{2} / 4}<0$.

We also need the following lemma, focusing only on some exponential functionals.

Lemma 7.3. Recall that $F^{ \pm}$and $G^{+}$are defined in (1.6) and (1.7). For all $0<$ $\zeta \leq 1$ and $0<\varepsilon<1$, for $h$ large enough,

$$
\begin{aligned}
& P\left[e^{(1-\varepsilon) \zeta h} \leq F^{+}(\zeta h) \leq e^{(1+\varepsilon) \zeta h}\right] \geq 1-4 e^{-\kappa \varepsilon \zeta h / 2}, \\
& P\left[F^{-}(h) \geq e^{-\varepsilon h}\right] \geq 1-e^{-\left(c_{-}\right) \varepsilon^{2} h^{2}}, \\
& P\left[G^{+}(\alpha h, h) \leq b(h) e^{h}\right] \geq 1-C_{+}[b(h)]^{-\kappa}, \quad 0<\alpha<1, b(h)>0 .
\end{aligned}
$$

Proof: By Markov inequality and the last line of Lemma 7.1,

$$
P\left[F^{+}(\zeta h)>e^{(1+\varepsilon) \zeta h}\right] \leq C_{10} e^{-\varepsilon \zeta h} \leq e^{-\kappa \varepsilon \zeta h / 2}
$$

for large $h$. For the lower bound, we have by Andreoletti and Devulder (2015, eq. (2.29)) for large $h$,

$$
P\left[F^{+}(\zeta h) \geq e^{(1-\varepsilon) \zeta h}\right] \geq 1-3 e^{-\kappa \varepsilon \zeta h / 2} .
$$

These two inequalities prove (7.8). For (7.9), first $F^{-}(h) \geq e^{-\varepsilon h} \tau^{W_{\kappa}^{\uparrow}}(\varepsilon h)$, and using $(7.7), \tau^{W_{\kappa}^{\uparrow}}(\varepsilon h) \geq 1$ with a probability larger than $1-e^{-\left(c_{-}\right) \varepsilon^{2} h^{2}}$, which proves $(7.9)$. Finally, notice that in law $G^{+}(\alpha h, h) \leq e^{h} \int_{0}^{+\infty} e^{W_{\kappa}(x)} \mathrm{d} x=e^{h} A_{\infty}$. By Dufresne (2000), $2 / A_{\infty}$ is a gamma variable of parameter $(\kappa, 1)$, and so has a density equal to $e^{-x} x^{\kappa-1} \mathbb{1}_{\mathbb{R}_{+}}(x) / \Gamma(\kappa)$, which leads to $(7.10)$.

The following lemma is exactly Lemma 4.3 in Andreoletti and Devulder (2015) which proof can be found in that paper.

Lemma 7.4. Let $(B(s), s \in \mathbb{R})$ be a standard two-sided Brownian motion. For every $0<\varepsilon<1,0<\delta<1$ and $x>0$,

$$
\begin{aligned}
& \mathbb{P}\left(\sup _{u \in[-\delta, \delta]}\left|\mathcal{L}_{B}\left(\tau^{B}(1), u\right)-\mathcal{L}_{B}\left(\tau^{B}(1), 0\right)\right|>\varepsilon \mathcal{L}_{B}\left(\tau^{B}(1), 0\right)\right) \leq C_{+} \frac{\delta^{1 / 6}}{\varepsilon^{2 / 5}}, \\
& \mathbb{P}\left(\sup _{u \in[0,1]} \mathcal{L}_{B}\left(\tau^{B}(1), u\right) \geq x\right) \leq 4 e^{-x / 2} \\
& \mathbb{P}\left(\sup _{u \leq 0} \mathcal{L}_{B}\left(\tau^{B}(1), u\right) \geq x\right) \leq 4 / x .
\end{aligned}
$$

The next lemma says that with large probability, a 2-dimensional squared Bessel Process is bounded by some deterministic function. This lemma may be of independent interest. 
Lemma 7.5. Let $\left(Q_{2}(u), u \geq 0\right)$ be a Bessel process of dimension 2, starting from 0 , and two functions $a($.$) and k($.$) from (0,+\infty)$ to $(0,+\infty)$, having limit $+\infty$ on $+\infty$. We have for large $t$,

$$
P\left(\forall u \in(0, k(t)], Q_{2}^{2}(u) \leq 2 e[a(t)+4 \log \log [e k(t) / u]] u\right) \geq 1-C_{+} \exp [-a(t) / 2] .
$$

Proof: We consider for $t>0$ and $i \in \mathbb{N}$,

$$
\mathcal{A}_{1, i}:=\left\{\sup _{\left[k(t) / e^{i+1}, k(t) / e^{i}\right]} Q_{2}^{2} \leq 2 \frac{k(t)}{e^{i}}[a(t)+4 \log (i+1)]\right\}, \quad \mathcal{A}_{2}:=\bigcap_{i=0}^{\infty} \mathcal{A}_{1, i} .
$$

We recall that there exist two standard independent Brownian motions $\left(B_{1}(u), u \geq\right.$ $0)$ and $\left(B_{2}(u), u \geq 0\right)$ such that $\left(Q_{2}^{2}(u), u \geq 0\right)$ is equal in law to $\left(B_{1}^{2}(u)+\right.$ $\left.B_{2}^{2}(u), u \geq 0\right)$. So for $i \in \mathbb{N}$,

$$
\begin{aligned}
P\left(\overline{\mathcal{A}}_{1, i}\right) & \leq 2 P\left(\sup _{\left[k(t) / e^{i+1}, k(t) / e^{i}\right]} B_{1}^{2}>k(t) e^{-i}[a(t)+4 \log (i+1)]\right) \\
& \leq 4 P\left(\sup _{\left[0, k(t) / e^{i}\right]} B_{1}>\sqrt{k(t) e^{-i}[a(t)+4 \log (i+1)]}\right) \\
& =4 P\left(\left|B_{1}(1)\right|>\sqrt{a(t)+4 \log (i+1)}\right) \\
& \leq 8 \exp [-a(t) / 2-2 \log (i+1)]
\end{aligned}
$$

for large $t$ so that $a(t) \geq 1$, by scaling, and since $B_{1} \stackrel{\text { 黑 }}{=}-B_{1}, \sup _{[0,1]} B_{1} \stackrel{\text { 近 }}{=}\left|B_{1}(1)\right|$ and $P\left(B_{1}(1) \geq x\right) \leq e^{-x^{2} / 2}$ for $x \geq 1$. Consequently for large $t$,

$$
P\left(\overline{\mathcal{A}}_{2}\right) \leq \sum_{i=0}^{\infty} P\left(\overline{\mathcal{A}}_{1, i}\right) \leq 8 \exp [-a(t) / 2] \sum_{i=0}^{\infty} \frac{1}{(i+1)^{2}}=C_{+} \exp [-a(t) / 2] .
$$

Now, let $0<u \leq k(t)$. There exists $i \in \mathbb{N}$ such that $k(t) / e^{i+1}<u \leq k(t) / e^{i}$. We have, $e^{i} \leq k(t) / u$, so $e^{i+1} \leq e k(t) / u$ and then $\log (i+1) \leq \log \log [e k(t) / u]$. Consequently on $\mathcal{A}_{2}$,

$$
Q_{2}^{2}(u) \leq 2\left(k(t) / e^{i}\right)[a(t)+4 \log (i+1)] \leq 2 e u[a(t)+4 \log \log [e k(t) / u]] .
$$

This, combined with (7.14), proves the lemma.

We also need some estimates on the local time of $B$ at a given coordinate $y \in \mathbb{R}$ at the inverse of the local time of $B$ at 0 . Recall that $\sigma_{B}(r, y)=\inf \{s>$ $\left.0, \mathcal{L}_{B}(s, y)>r\right\}$ for $r>0, y \in \mathbb{R}$. By the second Ray-Knight Theorem, the processes $\left(\mathcal{L}_{B}\left(\sigma_{B}(r, 0), y\right), y \in \mathbb{R}_{+}\right)$and $\left(\mathcal{L}_{B}\left(\sigma_{B}(r, 0),-y\right), y \in \mathbb{R}_{+}\right)$are two independent squared Bessel processes of dimension 0 starting at $r$. The following lemma is proved in Talet (2007, Lemma 3.1); the results are stated for a Bessel process but are actually true for a squared Bessel process; see also Diel (2011, Lemma 2.3).

Lemma 7.6. We denote by $\left(Q_{0}(y), y \geq 0\right)$ the square of a 0 -dimensional Bessel process starting at 1 . Let $M>0, u>0$ and $v>0$. Then,

$$
\begin{aligned}
& P\left(\sup _{0 \leq y \leq v}\left|Q_{0}(y)-1\right| \geq u\right) \leq 4 \frac{\sqrt{(1+u) v}}{u} \exp \left[-u^{2} /(8(1+u) v)\right], \\
& P\left(\sup _{y \geq 0} Q_{0}(y) \geq M\right)=1 / M .
\end{aligned}
$$




\section{Acknowledgements}

We are grateful to an anonymous referee for his or her very careful reading of the paper, and for comments that helped us improve the clarity and the presentation of the paper.

\section{References}

O. Adelman and N. Enriquez. Random walks in random environment: what a single trajectory tells. Israel J. Math. 142, 205-220 (2004). MR2085716.

P. Andreoletti. On the estimation of the potential of Sinai's RWRE. Braz. J. Probab. Stat. 25 (2), 121-144 (2011). MR2793922.

P. Andreoletti and A. Devulder. Localization and number of visited valleys for a transient diffusion in random environment. Electron. J. Probab. 20, no. 56, 58 (2015). MR3354616.

P. Andreoletti and R. Diel. Limit law of the local time for Brox's diffusion. J. Theoret. Probab. 24 (3), 634-656 (2011). MR2822476.

P. Andreoletti and R. Diel. DNA unzipping via stopped birth and death processes with unknown transition probabilities. Appl. Math. Res. Express. AMRX (2), 184-208 (2012). MR2982773.

P. Andreoletti, D. Loukianova and C. Matias. Hidden Markov model for parameter estimation of a random walk in a Markov environment. ESAIM Probab. Stat. 19, 605-625 (2015). MR3433429.

J. Bertoin. Lévy processes, volume 121 of Cambridge Tracts in Mathematics. Cambridge University Press, Cambridge (1996). ISBN 0-521-56243-0. MR1406564.

P. Billingsley. Convergence of probability measures. Wiley Series in Probability and Statistics: Probability and Statistics. John Wiley \& Sons, Inc., New York, second edition (1999). ISBN 0-471-19745-9. MR1700749.

A. N. Borodin and P. Salminen. Handbook of Brownian motion-facts and formulae. Probability and its Applications. Birkhäuser Verlag, Basel, second edition (2002). ISBN 3-7643-6705-9. MR1912205.

A. Bovier. Extremes, sums, Lévy processes, and ageing. Lecture (2010). https://wt.iam.uni-bonn.de/fileadmin/WT/Inhalt/people/Anton Bovier/lecture-notes/levy.pdf.

Th. Brox. A one-dimensional diffusion process in a Wiener medium. Ann. Probab. 14 (4), 1206-1218 (1986). MR866343.

D. Cheliotis. Localization of favorite points for diffusion in a random environment. Stochastic Process. Appl. 118 (7), 1159-1189 (2008). MR2428713.

F. Comets, M. Falconnet, O. Loukianov and D. Loukianova. Maximum likelihood estimator consistency for recurrent random walk in a parametric random environment with finite support $(2016+)$. To appear in Stochastic Process. Appl.

F. Comets, M. Falconnet, O. Loukianov, D. Loukianova and C. Matias. Maximum likelihood estimator consistency for a ballistic random walk in a parametric random environment. Stochastic Process. Appl. 124 (1), 268-288 (2014). MR3131294.

A. Dembo, N. Gantert, Y. Peres and Z. Shi. Valleys and the maximum local time for random walk in random environment. Probab. Theory Related Fields 137 (3-4), 443-473 (2007). MR2278464. 
A. Devulder. The maximum of the local time of a diffusion process in a drifted Brownian potential (2016+). To appear in Séminaire de Probabilités XLVIII.

R. Diel. Almost sure asymptotics for the local time of a diffusion in Brownian environment. Stochastic Process. Appl. 121 (10), 2303-2330 (2011). MR2822778.

D. Dufresne. Laguerre series for Asian and other options. Math. Finance 10 (4), 407-428 (2000). MR1785163.

N. Enriquez, C. Sabot and O. Zindy. Aging and quenched localization for onedimensional random walks in random environment in the sub-ballistic regime. Bull. Soc. Math. France 137 (3), 423-452 (2009a). MR2574090.

N. Enriquez, C. Sabot and O. Zindy. A probabilistic representation of constants in Kesten's renewal theorem. Probab. Theory Related Fields 144 (3-4), 581-613 (2009b). MR2496443.

A. Faggionato. The alternating marked point process of $h$-slopes of drifted Brownian motion. Stochastic Process. Appl. 119 (6), 1765-1791 (2009). MR2519344.

M. Falconnet, D. Loukianova and C. Matias. Asymptotic normality and efficiency of the maximum likelihood estimator for the parameter of a ballistic random walk in a random environment. Math. Methods Statist. 23 (1), 1-19 (2014). MR3189262.

W. Feller. An introduction to probability theory and its applications. Vol. II. Second edition. John Wiley \& Sons, Inc., New York-London-Sydney (1971). MR0270403.

N. Gantert, Y. Peres and Z. Shi. The infinite valley for a recurrent random walk in random environment. Ann. Inst. Henri Poincaré Probab. Stat. 46 (2), 525-536 (2010). MR2667708.

N. Gantert and Z. Shi. Many visits to a single site by a transient random walk in random environment. Stochastic Process. Appl. 99 (2), 159-176 (2002). MR1901151.

Y. Hu, Z. Shi and M. Yor. Rates of convergence of diffusions with drifted Brownian potentials. Trans. Amer. Math. Soc. 351 (10), 3915-3934 (1999). MR1637078.

K. Kawazu and H. Tanaka. A diffusion process in a Brownian environment with drift. J. Math. Soc. Japan 49 (2), 189-211 (1997). MR1601361.

H. Kesten, M. V. Kozlov and F. Spitzer. A limit law for random walk in a random environment. Compositio Math. 30, 145-168 (1975). MR0380998.

J. Neveu and J. Pitman. Renewal property of the extrema and tree property of the excursion of a one-dimensional Brownian motion. In Séminaire de Probabilités, XXIII, volume 1372 of Lecture Notes in Math., pages 239-247. Springer, Berlin (1989). MR1022914.

S. I. Resnick. Point processes, regular variation and weak convergence. Adv. in Appl. Probab. 18 (1), 66-138 (1986). MR827332.

D. Revuz and M. Yor. Continuous martingales and Brownian motion, volume 293 of Grundlehren der Mathematischen Wissenschaften [Fundamental Principles of Mathematical Sciences]. Springer-Verlag, Berlin, third edition (1999). ISBN 3540-64325-7. MR1725357.

S. Schumacher. Diffusions with random coefficients. In Particle systems, random media and large deviations (Brunswick, Maine, 1984), volume 41 of Contemp. Math., pages 351-356. Amer. Math. Soc., Providence, RI (1985). MR814724.

Z. Shi. A local time curiosity in random environment. Stochastic Process. Appl. 76 (2), 231-250 (1998). MR1642673.

D. S. Silvestrov. Convergence in Skorokhod $J$-topology for compositions of stochastic processes. Theory Stoch. Process. 14 (1), 126-143 (2008). MR2479713. 
A. Singh. Rates of convergence of a transient diffusion in a spectrally negative Lévy potential. Ann. Probab. 36 (1), 279-318 (2008). MR2370605.

M. Talet. Annealed tail estimates for a Brownian motion in a drifted Brownian potential. Ann. Probab. 35 (1), 32-67 (2007). MR2303943.

H. Tanaka. Limit theorems for a Brownian motion with drift in a white noise environment. Chaos Solitons Fractals 8 (11), 1807-1816 (1997). MR1477261.

G. Véchambre. Path decomposition of a spectrally negative Lévy process, and local time of a diffusion in this environment. ArXiv Mathematics e-prints (2016). arXiv: 1605.05084.

W. Whitt. Stochastic-process limits. Springer Series in Operations Research. Springer-Verlag, New York (2002). ISBN 0-387-95358-2. An introduction to stochastic-process limits and their application to queues. MR1876437.

O. Zeitouni. Random walks in random environment. In Lectures on probability theory and statistics, volume 1837 of Lecture Notes in Math., pages 189-312. Springer, Berlin (2004). MR2071631. 